\title{
A class of recursion operators on a tangent bundle
}

Fien Vermeire

Proefschrift ingediend aan de Faculteit Wetenschappen

tot het behalen van de graad van

Doctor in de Wetenschappen: Wiskunde

Promotor: Prof. Dr. W. Sarlet Co-promotor: Prof. Dr. M. Crampin

Universiteit Gent

Faculteit Wetenschappen

Vakgroep Wiskundige Natuurkunde en Sterrenkunde

Academiejaar 2006-2007

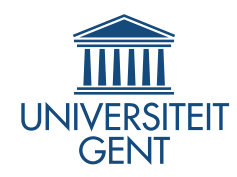





\section{Voorwoord}

In dit voorwoord wil ik iedereen bedanken die rechtstreeks of onrechtstreeks heeft bijgedragen tot het totstandkomen van dit proefschrift.

In de eerste plaats wil ik mijn promotor Willy Sarlet bedanken voor de hulp, zijn geduld, voor het nalezen en verbeteren van mijn verhandeling. Mijn dank gaat ook uit naar Frans Cantrijn en Mike Crampin voor hun interesse en goede raad. En ook mijn collega's mag ik niet vergeten: in het bijzonder bedank ik Joris, Tom , Bavo, Jos en David voor de gezellige sfeer, de interessante babbels en de $\mathrm{LTT}_{\mathrm{E}} \mathrm{X}$-hulp.

Verder wil ik ook mijn familie en vrienden bedanken. Vooral mijn ouders ben ik heel dankbaar voor hun jarenlange steun en hun luisterend oor. Tot slot dank ik mijn lieve echtgenoot Dieter omdat hij altijd voor mij klaarstaat, voor de bemoedigende woorden en al zijn hulp.

Fien Vermeire, 28 augustus 2006 



\section{Contents}

$\begin{array}{ll}\text { Introduction } & 1\end{array}$

1 Bi-Hamiltonian systems and separability $\quad 7$

1.1 Poisson manifolds . . . . . . . . . . . . . . . . . . 7

1.2 Bi-Hamiltonian systems $\ldots \ldots \ldots \ldots$

1.2.1 Recursion operators . . . . . . . . . . . . . . 14

1.2.2 Poisson-Nijenhuis structures . . . . . . . . . . . . 17

1.3 Separability of the Hamilton-Jacobi equation . . . . . . . . 21

1.3.1 Liouville systems . . . . . . . . . . . . . . . 22

1.3 .2 Stäckel systems . . . . . . . . . . . . . . . . . 22

1.3.3 Levi-Civita conditions . . . . . . . . . . . . . 23

1.3.4 Eisenhart's theorem . . . . . . . . . . . . . 24

1.3.5 Benenti systems . . . . . . . . . . . . 25

1.3.6 Separability for bi-Hamiltonian systems . . . . . . 26

2 Special conformal Killing tensors $\quad 29$

2.1 Killing tensors . . . . . . . . . . . . . . . . . . . . 29

2.2 Conformal Killing tensors _ . . . . . . . . . . . . . . 30

2.3 Special conformal Killing tensors . . . . . . . . . . . . . 31

2.4 SCK tensors and separability . . . . . . . . . . . . 32

2.5 SCK tensors and cofactor systems . . . . . . . . . 33

2.6 SCK tensors and projective equivalence . . . . . . . . 37

2.7 SCK tensors and Poisson-Nijenhuis structures . . . . . . . . 38 
3.1 Calculus along the tangent bundle projection . . . . . . . 41

3.1.1 The Riemannian case . . . . . . . . . . . . . 46

3.2 The construction of a type $(1,1)$ tensor field $R \ldots 46$

3.2.1 The Darboux-Nijenhuis coordinates of $R \ldots \ldots$. . . 51

3.3 Investigation of $\mathcal{L}_{\Gamma} R \ldots \ldots \ldots \ldots \ldots$

3.4 When the base manifold is Riemannian . . . . . . . . 56

4 More general recursion operators $\quad 63$

4.1 R-tensors associated to a $(1,1)$ tensor field along $\tau \ldots$. . . 63

4.2 Invariant R-tensors . . . . . . . . . . . . 71

4.2 .1 Alternative Lagrangians . . . . . . . . . . . . 72

4.3 The Nijenhuis torsion of $\widetilde{J}$ and $R \ldots \ldots \ldots$

4.4 Illustrative examples . . . . . . . . . . . . . . . . 82

4.5 The case of interest when $\mathcal{L}_{\Gamma} R$ does not vanish $\ldots \ldots$. . 86

5 Application: R-tensors related to a Finsler metric $\quad 89$

5.1 Finsler spaces . . . . . . . . . . . . . . . . . . . . . . 89

5.2 R-tensors in the Finsler case . . . . . . . . . . . . . . 92

5.3 Generalisation of the SCK condition . . . . . . . . 95

5.4 A recursive scheme of first integrals . . . . . . . . . . 102

6 Projective equivalence of Finsler metrics 109

6.1 Linear connection along the tangent bundle projection . . . . 110

6.2 Projective equivalence of sprays . . . . . . . . . . . 115

6.3 Projective equivalence with the canonical spray on a Finsler space . . . . . . . . . . . . . . 117

6.4 Projective equivalence of two Finsler metrics . . . . . . 118

6.5 Nijenhuis torsion condition on $L \ldots \ldots \ldots \ldots$

6.6 Example with a Randers metric . . . . . . . . . . . . 125 
Bibliography

Index

131

Samenvatting

133 



\section{Introduction}

The general framework wherein this thesis fits, is the study of integrable Hamiltonian systems. This subject started with a famous result of Liouville - which says that a $2 n$-dimensional Hamiltonian system is completely integrable if there are $n$ first integrals in involution - and is still an active area of research. A special role within this theory is played by systems with a bi-Hamiltonian structure, i.e. systems which are Hamiltonian with respect to two compatible Poisson structures. In such a case one can find a set of first integrals which are in involution with respect to both Poisson structures. There is, moreover, a link between bi-Hamiltonian systems (in particular Poisson-Nijenhuis structures) and the theory of separability of the Hamilton-Jacobi equation. More information about such topics can be found in the first chapter of this work.

Consider a Riemannian manifold $(Q, g)$ and a type $(1,1)$ tensor $J$ on $Q$. A well known way to construct a bi-Hamiltonian structure goes as follows. The canonical symplectic structure $\omega=d \theta$ on the cotangent bundle determines a first Poisson bracket. By means of the tensor $J$ one can construct a second 2-form $\omega_{1}=d(J \theta)$ on $T^{*} Q$ with associated Poisson bracket. The tensor $R$ determined by

$$
i_{R(\xi)} \omega=i_{\xi} \omega_{1}
$$

with $\xi$ an arbitrary vector field on $T^{*} Q$, is the complete lift of $J$ to $T^{*} Q$. If the Nijenhuis torsion of $J$ vanishes, then so will the Nijenhuis torsion of $R$. In that case $R$ is the recursion operator of a Poisson-Nijenhuis structure determined by $\omega$ and $J$.

One can easily investigate under what circumstances $R$ will be invariant, i.e. will be a recursion operator for generating symmetries of the kinetic energy Hamiltonian system associated to $g$. But we will focus more on a special situation of non-invariance of $R$ which is related to a so called gauged bidifferential calculus. To be precise, an interesting situation, studied in [8], is the case that the tensor $J$ satisfies equations of the form

$$
J_{i j \mid k}=\frac{1}{2}\left(g_{j k} \alpha_{i}+g_{i k} \alpha_{j}\right)
$$


where $\mid k$ denotes the covariant derivative, $\alpha_{i}=\frac{\partial f}{\partial q^{i}}$ and $f=\operatorname{tr} J$. Since it then follows that

$$
J_{i j \mid k}+J_{j k \mid i}+J_{k i \mid j}=g_{i j} \alpha_{k}+g_{j k} \alpha_{i}+g_{k i} \alpha_{j},
$$

$J$ is conformal Killing and was therefore called a special conformal Killing tensor in [9]. If $J$ has also simple eigenvalues, it is called a Benenti tensor, after S. Benenti who was the first to investigate such tensors.

This class of tensors has very interesting properties. First of all, it can be calculated that the Nijenhuis torsion of a special conformal Killing tensor automatically vanishes, giving rise to two compatible Poisson structures in the way described before. Another important property is that the cofactor tensor of a special conformal Killing tensor is always a Killing tensor. So it defines a quadratic first integral. A set of first integrals in involution can be found if we consider the coefficients of the different powers of $s$ of the cofactor tensor of the special conformal Killing tensor $J+s I$, where $s$ is a constant (see for example [10]).

Special conformal Killing tensors arise also in a number of other contexts. One example is the theory of cofactor systems and cofactor pair systems. This subject was studied by H. Lundmark ([30]) on an Euclidean space and was extended by M. Crampin and W. Sarlet to the case of a general Riemannian manifold ([9]). It is shown that a cofactor system can be interpreted as a non-conservative Lagrangian system which admits a quasi-Hamiltonian representation with respect to a Poisson bracket associated to a type $(1,1)$ tensor $L$, where $L$ has to be a special conformal Killing tensor. A cofactor pair system has a bi-quasi-Hamiltonian structure and is completely integrable.

Another application can be found in the study of projectively or geodesically equivalent Riemannian metrics, i.e. metrics which have the same geodesics as point sets. The tensor $L$ which then links the two metrics, again has to be a special conformal Killing tensor. This topic can be related to a quasi-bi-Hamiltonian system and will also lead to a set of first integrals in involution (see for example [11]).

A principal aim of our research is to find a generalisation of the theory of special conformal Killing tensors from a Riemannian manifold to a general tangent bundle with a regular Lagrangian, in particular to a Finsler manifold. Therefore, it is important to understand how the results of the applications we have just mentioned and which are developed in a cotangent bundle environment, can be obtained in a natural way by tangent bundle 
techniques. In a major part of the thesis, certainly so far as intrinsic and coordinate free calculations are concerned, we make use of the calculus along the tangent bundle projection, developed in [36] and [37]. But coordinate calculations will be used where convenient.

In our investigation on a Finlser manifold, or more generally on a Lagrangian manifold, it will be natural to assume that the tensor $J$ we start from, is velocity dependent. Technically this means that $J$ will be a tensor field along the tangent bundle projection. In the particular situation that we consider a Lagrangian coming from a Finsler metric, it will be natural to assume that $J$ is homogeneous of degree zero in the velocities.

Although a number of results of the Riemannian situation are also valid in the Finslerian case, there will be a lot of complications. By far, the main problem in our attempt to come to generalisations has to do with the Nijenhuis torsion of our generalised R-tensor. Also, Nijenhuis-type tensors associated to what we will identify as of the generalised special conformal Killing tensor will not automatically vanish.

The scheme of the thesis is as follows. In the first chapter we discuss the general setting. First we recall briefly the theory of bi-Hamiltonian systems which was discovered by F. Magri in 1978 while studying evolution equations like the Korteweg-De Vries equation. In particular we consider the PoissonNijenhuis structures and associated bi-differential calculus. Some important results of the separability theory will also be recalled briefly in this chapter: for example the definition of Stäckel separability and Eisenhart's theorem about the relationship between separability and Killing tensors.

Chapter 2 is devoted to the definition and properties of special conformal Killing tensors. We discuss in some more detail the different situations where such tensors arise.

The presentation of our own research results starts in chapter 3. First we give a review of the calculus along the tangent bundle projection. This gives us an elegant way to translate the results of the theory about PoissonNijenhuis structures, as recalled in chapter 2, into a tangent bundle version. We shall see that this different way of approaching the subject offers some new insights. In fact, some results will be valid for arbitrary Lagrangians. We will obtain an alternative intrinsic expression for a type (1,1) tensor on a Riemannian manifold $(Q, g)$ to be a special conformal Killing tensor. Namely, a symmetric type $(1,1)$ tensor $J$ is a special conformal Killing tensor 
if it satisfies

$$
g(\nabla J(X), Y)=\frac{1}{2}[g(X, \mathbf{T}) \alpha(X)+g(Y, \mathbf{T}) \alpha(Y)]
$$

with $X$ and $Y$ arbitrary vector fields on $Q, \mathbf{T}=u^{i} \frac{\partial}{\partial q^{i}}$ and $\nabla$ the dynamical covariant derivative associated to the Euler-Lagrange equations of the kinetic energy defined by $g$.

Once we understand in detail how the tangent bundle approach works, we can begin with the generalisation. In chapter 4, we start from a general Lagrangian system and a type $(1,1)$ tensor $J$ which depends also on the velocities. We use a relation of type (1) to define R-tensors in a more general way and we find that the structure of such an $R$ is characterised by two type $(1,1)$ tensors $K$ and $U$ along the tangent bundle projection. Especially the tensor $K$ will play an important role in this theory and it has a lot of interesting properties. Invariance of $R$ under the given Lagrangian flow implies, for example, that $K$ has to be symmetric and parallel and has to commute with the Jacobi endomorphism. This topic is also related to the theory of alternative Lagrangian systems and we will illustrate this theory on some simple systems. In the same chapter we will also investigate the requirements for the Nijenhuis torsion of $R$ to vanish. As said before, this investigation requires some hard and long calculations.

If we restrict ourselves to the case that we work on a Finsler manifold (see chapter 5), the conditions for $R$ to be invariant and for the Nijenhuis torsion of $R$ to be zero will simplify. Moreover, the same construction that led in chapter 3 to the special conformal Killing condition, will bring us here to one of the principal expressions in this thesis, namely:

$$
g(\nabla K(X), Y))=\frac{1}{2}[g(X, \mathbf{T}) \alpha(Y)+g(Y, \mathbf{T}) \alpha(X)]
$$

where $g$ is a Finsler metric and $\alpha$ is a semi-basic 1-form. Also in this case the cofactor tensor of a tensor $K$ which satisfies (2) determines a first integral. In fact we can find a whole set of (non-quadratic) first integrals and we do this via a different procedure than the one which relies on the cofactor tensor of $K+s I$. It has as the advantage that $K$ doesn't have to be non-singular.

Expression (2) will reappear in chapter 6 , where we investigate projective equivalence of two Finsler metrics. In section 6.4, we start from a Finsler manifold $(Q, g)$ and a non-singular symmetric type $(1,1)$ tensor $L$ along the projection, homogeneous of degree 0 . We define a type $(0,2)$ tensor $\widetilde{g}$ and investigate under which circumstances $\widetilde{g}$ is a Finsler metric projectively 
equivalent to $g$. The fact that $\widetilde{g}$ has to be a Hessian gives already a first condition. The projective equivalence requires that $L$ satisfies (2) with an extra condition on $\alpha$. This last condition, by the way, holds automatically in the Riemannian case as a consequence of the vanishing of the Nijenhuis torsion. So, in the subsequent section we investigate the influence of Nijenhuis-type conditions on $L$ on the extra requirement on $\alpha$.

Some parts of this thesis have already been published. The results of chapter 3 have appeared in [44]. The generalised theory about the recursion properties (see chapter 4 and chapter 5) can be found in [52]. 


\section{Chapter 1}

\section{Bi-Hamiltonian systems and separability}

This first introductionary chapter is to explain the general context in which this thesis is situated. We will give a historical overview of the bi-Hamiltonian approach to Liouville integrability and discuss also separable systems. But first we recall some definitions, mainly to fix notations and terminology. Section 1.1 is based on the book [27] by Libermann and Marle.

\subsection{Poisson manifolds}

Let $M$ be a smooth manifold of dimension $m . C^{\infty}(M)$ denotes the set of smooth functions on $M . \mathcal{X}(M)$ and $\mathcal{X}^{*}(M)$ are respectively the set of vector fields and one forms on $M$.

Definition 1. A Poisson manifold $M$ is a differentiable manifold equipped with a Poisson structure. This means that there exists a bilinear map

$$
\begin{array}{cl}
C^{\infty}(M) \times C^{\infty}(M) & \rightarrow C^{\infty}(M) \\
(f, g) & \mapsto\{f, g\}
\end{array}
$$

called the Poisson bracket, that satisfies the following properties:

1. it is skew symmetric,

$$
\{f, g\}=-\{g, f\}
$$

2. it satisfies the Jacobi identity,

$$
\{f,\{g, h\}\}+\{g,\{h, f\}\}+\{h,\{f, g\}\}=0 ;
$$

3. it satisfies the Leibniz rule,

$$
\{f, g h\}=g\{f, h\}+\{f, g\} h
$$

for all $f, g, h \in C^{\infty}(M)$. 
On every Poisson manifold $M$ there exists a unique bivector field $\Pi$ such that for every pair $(f, g)$ of functions on $M$,

$$
\{f, g\}=\Pi(d f, d g) .
$$

This bivector field $\Pi$ is called the Poisson tensor associated to the bracket \{\} . The Jacobi condition of the above definition is equivalent to saying that the Schouten bracket of $\Pi$ with itself vanishes: $[\Pi, \Pi]=0$.

The Poisson map $P: \mathcal{X}^{*}(M) \rightarrow \mathcal{X}(M)$ is defined by

$$
\Pi(\alpha, \beta)=i_{P(\alpha)} \beta \quad \text { for } \quad \alpha, \beta \in \mathcal{X}^{*}(M) .
$$

Definition 2. $k$ functions $f_{k}$ on a Poisson manifold $M$ are said to be in involution if they pairwise commute with respect to the Poisson bracket, i.e. each pair of functions $f_{i}, f_{j}$ satisfies $\left\{f_{i}, f_{j}\right\}=0$.

A function $h \in C^{\infty}(M)$ which is in involution with each other function on $M$ is called a Casimir function. In terms of the Poisson map $P$, a Casimir function is a function $h$ such that $P(d h)=0$.

Definition 3. Let $(M,\{\})$ be a Poisson manifold and $f \in C^{\infty}(M)$. The Hamiltonian vector field $X_{f}$ associated with $f$, is the unique vector field on $M$ such that

$$
X_{f}(g)=\{f, g\}=P(d f)(g) \quad \forall g \in C^{\infty}(M) .
$$

In the case that we have a symplectic manifold $(M, \omega)$, we can define a non-degenerate Poisson structure as follows:

$$
\{f, g\}=\omega\left(X_{f}, X_{g}\right)
$$

where $i_{X_{f}} \omega=-d f$.

Definition 4. Let $(M,\{\})$ be a Poisson manifold of dimension $m=2 n$. A Hamiltonian system $(M,\{\}, H)$ on this manifold is completely integrable (in the Liouville-Arnol'd sense) if it admits n functionally independent integrals of motion $I_{i}$ which are in involution: $\left\{I_{i}, I_{j}\right\}=0$

Liouville proved in 1855 that any integrable system can be solved by quadratures [29].

To end this section, note that one can also consider infinite-dimensional Poisson manifolds. The theory is similar except for some conversions: 
- we have to replace functions by functionals;

- the exterior derivative $d$ has to be replaced by the variational derivative $\frac{\delta}{\delta u} \cdot \frac{\delta}{\delta u}$ acts on any functional $\mathcal{F}(u)=\int f\left(u, u_{x}, u_{x x}, \ldots\right) d x$ as follows

$$
\frac{\delta \mathcal{F}}{\delta u}=\frac{\partial f}{\partial u}-D_{x}\left(\frac{\partial f}{\partial u_{x}}\right)+D_{x}^{2}\left(\frac{\partial f}{\partial u_{x x}}\right) \ldots ;
$$

- The Poisson map $P$ becomes in this case a differential operator $\mathcal{P}$.

$\mathcal{P}$ defines a Poisson bracket

$$
\{\mathcal{F}, \mathcal{G}\}=\int \frac{\delta \mathcal{F}}{\delta u} \mathcal{P} \frac{\delta \mathcal{G}}{\delta u} d x
$$

where $\mathcal{F}$ and $\mathcal{G}$ are functionals. A Hamiltonian system of evolution equations takes then the form

$$
u_{t}=\mathcal{P}\left(\frac{\delta \mathcal{K}}{\delta u}\right)
$$

In the next section we will use this formalism in an interesting well-known example, namely the Korteweg De Vries equation.

\subsection{Bi-Hamiltonian systems}

The theory of bi-Hamiltonian systems originated in the study of evolution equations, in particular in the study of the Korteweg De Vries equation. The KdV equation

$$
u_{t}=\frac{1}{4}\left(u_{x x x}-6 u u_{x}\right) .
$$

was derived in 1895 by Korteweg and De Vries and describes the famous observation of a solitary water wave, made by John Scott Russel in 1834. In 1965 the KdV equation reappeared in the work of Kruskal and Zabusky. They found that the solitary waves travelled 'through' each other with no change of shape or speed. That's why they introduced the term solitons for the solutions of the KdV equation. Two years later, in 1967, Gardner, Kruskal and Miura developed the Miura transformation and inverse scattering method and demonstrated the existence of an infinite number of conservation laws (i.e. expressions of the form $\operatorname{div} P$ which vanish for all solutions of the given system; $P$ denotes a $p$-tuple of functions $\left.\left(P_{1}\left(x, u^{(n)}\right), \ldots, P_{p}\left(x, u^{(n)}\right)\right)\right)$. 
The next breakthrough was in 1971, when Gardner and Faddeev and Zakharov showed the Hamiltonian structure of the KdV equation. Let us first suppose that $u$ is periodic in $x: u(0, t)=u(2 \pi, t)$. Then equation (1.1) can be written as follows

$$
u_{t}=\left[-2 D_{x}\right]\left[\frac{1}{8}\left(3 u^{2}-u_{x x}\right)\right]=\mathcal{P}_{0}\left[\frac{1}{8}\left(3 u^{2}-u_{x x}\right)\right]
$$

and the '1-form' $\alpha=\frac{1}{8}\left(3 u^{2}-u_{x x}\right)$ is the variational derivative $\frac{\delta}{\delta u}$ of $\mathcal{K}_{0}=\frac{1}{8} \int_{0}^{2 \pi}\left(u^{3}+\frac{1}{2} u_{x}^{2}\right) d x$. The operator $\mathcal{P}_{0}$ defines a map on functionals

$$
\{\mathcal{F}, \mathcal{G}\}_{0}=\int_{0}^{2 \pi} \frac{\delta \mathcal{F}}{\delta u} \mathcal{P}_{0} \frac{\delta \mathcal{G}}{\delta u} d x
$$

which is $\mathbb{R}$-bilinear, skew symmetric and satisfies the Jacobi-identity and the Leibniz rule. Therefore, \{\}$_{0}$ is said to be a Poisson bracket and we have an infinite-dimensional Hamiltonian system.

But this representation is not unique. In 1978 Magri [32] observed that the $\mathrm{KdV}$ equation can also be written as

$$
u_{t}=\left[-\frac{1}{2} D_{x}^{3}+2 u D_{x}+u_{x}\right]\left[-\frac{1}{2} u\right]=\mathcal{P}_{1}\left[-\frac{1}{2} u\right]
$$

with $-\frac{1}{2} u=\frac{\delta K_{1}}{\delta u}$ where $\mathcal{K}_{1}$ is given by $\mathcal{K}_{1}=-\int_{o}^{2 \pi} \frac{u^{2}}{4} d x$. The operator $\mathcal{P}_{1}$ defines a Poisson bracket too

$$
\{\mathcal{F}, \mathcal{G}\}_{1}=\int_{0}^{2 \pi} \frac{\delta \mathcal{F}}{\delta u} \mathcal{P}_{1} \frac{\delta \mathcal{G}}{\delta u} d x
$$

It turns out that this is compatible with \{\}$_{0}$, in the sense that every linear combination \{\}$_{1}-\lambda\{\}_{0}$ is still a Poisson bracket. The Poisson map is given by

$$
\mathcal{P}^{\lambda}=\mathcal{P}_{1}-\lambda \mathcal{P}_{0}=-\frac{1}{2} D_{x}^{3}+2(u+\lambda) D_{x}+u_{x} .
$$

For this reason the KdV equation is said to have a bi-Hamiltonian representation. In [32] Magri showed also that the property of having two Hamiltonian representations is not a specific feature of the KdV equations, but a general property for integrable soliton equations. Gel'fand and Dorfman transferred these ideas to the field of finite-dimensional Hamiltonian systems. By analogy, the definition of a finite-dimensional bi-Hamiltonian system can be formulated as follows. 
Definition 5. A bi-Hamiltonian manifold is a Poisson manifold endowed with a pair of compatible Poisson brackets \{\}$_{0}$ and \{\}$_{1}$. They are compatible if the pencil

$$
\{\}^{\lambda}:=\{\}_{1}-\lambda\{\}_{0}
$$

is also a Poisson bracket.

A vector field $X$ that can be written as $X=P_{0} d f=P_{1} d g$ (for 2 functions $f$ and $g$ ) is called a bi-Hamiltonian vector field.

Now we want to show how the bi-Hamiltonian point of view can be used to find the infinite sequence of conservation laws. But first we recall some terminology. Suppose that we have a conservation law of the form $D_{t} T+$ $D_{x} X=0$ where $T$ and $X$ are functions of $x, t, u$ and the derivatives of $u$ w.r.t $x$ and $t$. By integrating this we find $0=\int_{0}^{2 \pi} D_{t} T d x+\left.X\right|_{0} ^{2 \pi}=D_{t} \int_{0}^{2 \pi} T d x$. We call $T$ a conserved density and the functional $\int_{0}^{2 \pi} T d x$ is referred to as a conserved quantity.

To find conservation laws of the KdV equation one can compute the Casimir of \{\}$^{\lambda}$, in other words a functional $\mathcal{H}(\lambda)$ such that $\mathcal{P}^{\lambda}\left(\frac{\delta}{\delta u} \mathcal{H}(\lambda)\right)=0$. The simplest way to do this is to use the Miura transformation $u \rightarrow h$ on the class of periodic functions, implicitly given by

$$
h_{x}+h^{2}=u+z^{2} .
$$

where $\lambda=z^{2}$ is a parameter. Then it can be shown that the Casimir functional is given by

$$
\mathcal{H}(\lambda)=2 z \int_{0}^{2 \pi} h\left(u, u_{x}, u_{x x}, \ldots, z\right) d x .
$$

Now we express $h$ as function of $u$ and therefore we look at $h$ in the form of Laurent series $h=z+\sum_{l \geq 1} h_{l} z^{-l}$. By equating the coefficients of different powers of $z$ in (1.2), we find

$$
\begin{aligned}
& h_{1}=\frac{1}{2} u \\
& h_{2}=-\frac{1}{4} u_{x} \\
& h_{3}=\frac{1}{8}\left(u_{x x}-u^{2}\right) \\
& h_{4}=-\frac{1}{16}\left(u_{x x x}-4 u u_{x}\right) \\
& h_{5}=\frac{1}{32}\left(u_{x x x x}-6 u u_{x x}-5 u_{x}^{2}+2 u^{3}\right)
\end{aligned}
$$


and so on. Notice that all even coefficients $h_{2 l}$ are total $x$-derivatives and therefore they vanish by integrating between 0 and $2 \pi$. So, $\mathcal{H}(\lambda)=$ $\sum_{l \geq 0} \mathcal{H}_{2 l+1} \lambda^{-l}$ with

$$
\begin{aligned}
\mathcal{H}_{1} & =\int_{0}^{2 \pi} u d x \\
\mathcal{H}_{3} & =\frac{1}{4} \int_{0}^{2 \pi}\left(u_{x x}-u^{2}\right) d x=-\frac{1}{4} \int_{0}^{2 \pi} u^{2} d x=\mathcal{K}_{1} \\
\mathcal{H}_{5} & =\frac{1}{16} \int_{0}^{2 \pi}\left(u_{x x x x}-6 u u_{x x}-5 u_{x}^{2}+2 u^{3}\right) d x \\
& =\frac{1}{16} \int_{0}^{2 \pi}\left(2 u^{3}+u_{x}^{2}\right) d x=\mathcal{K}_{0}
\end{aligned}
$$

When we express that $\mathcal{H}$ is a Casimir of $\mathcal{P}_{\lambda}$, we find that $\mathcal{P}_{0} \frac{\delta}{\delta u} \mathcal{H}_{1}=0$ and that the coefficients $\mathcal{H}_{i}$ satisfy Lenard's recursion relations

$$
\mathcal{P}_{0} \frac{\delta}{\delta u} \mathcal{H}_{k+2}=\mathcal{P}_{1} \frac{\delta}{\delta u} \mathcal{H}_{k} \quad \text { for } \quad k=1,3,5, \ldots
$$

By repeatedly using this recursion relation, one can prove that the functionals $\mathcal{H}_{i}$ are all in involution with respect to \{\}$_{0}$ and \{\}$_{1}$ and are conserved quantities. For example

$$
\left\{\mathcal{H}_{3}, \mathcal{K}_{0}\right\}_{0}=\left\{\mathcal{H}_{3}, \mathcal{H}_{5}\right\}_{0}=\left\{\mathcal{H}_{3}, \mathcal{H}_{3}\right\}_{1}=0
$$

and the conservation law corresponding to $\mathcal{H}_{3}$ is $D_{x}\left(-\frac{1}{4} u^{3}+\frac{1}{8} u u_{x x}-\frac{1}{16} u_{x}^{2}\right)$ $+D_{t}\left(-\frac{1}{4} u^{2}\right)=0$.

Now we can write down the so called $K d V$ hierarchy which is defined by

$$
D_{t_{j}} u=\mathcal{P}_{1} \frac{\delta}{\delta u} \mathcal{H}_{j}=\mathcal{P}_{0} \frac{\delta}{\delta u} \mathcal{H}_{j+2}
$$

i.e.

$$
\begin{aligned}
& u_{t_{1}}=u_{x} \\
& u_{t_{3}}=\frac{1}{4}\left(u_{x x x}-6 u u_{x}\right) \quad(\mathrm{KdV} \text { equation }) \\
& u_{t_{5}}=\frac{1}{16}\left(u_{x x x x x}-10 u u_{x x x}-20 u_{x} u_{x x}+30 u^{2} u_{x}\right)
\end{aligned}
$$

Another way to find the KdV hierarchy and the conserved quantities, is to use the so called recursion operator $R=\mathcal{P}_{1} \mathcal{P}_{0}^{-1}=D_{x}^{2}-4 u-2 u_{x} D_{x}^{-1}$ *

\footnotetext{
* Remark that integral operator $\left(D_{x}\right)^{-1}$ is only defined on functions $F$ of the form $F=D_{x} G$
} 
Namely put $\mathcal{E}_{0}=\mathcal{P}_{0} \frac{\delta}{\delta u} \mathcal{K}_{1}$ and define recursively $\mathcal{E}_{n}=R \mathcal{E}_{n-1}$ for $n \geq 1$. Then the $u_{t_{2 n+1}}=\mathcal{E}_{n}$ form the KdV hierarchy (for example $u_{t_{3}}=\mathcal{E}_{1}$ is the $\mathrm{KdV}$ equation) and for each $n \geq 1, u_{t}=\mathcal{E}_{n}=\mathcal{P}_{0} \frac{\delta}{\delta u} \mathcal{H}_{n+1}=\mathcal{P}_{1} \frac{\delta}{\delta u} \mathcal{H}_{n}$ is a bi-Hamiltonian system and the Hamiltonian functionals $\mathcal{H}_{n}$ are conserved.

The KdV hierarchy is important for constructing solutions of the KdV equation, for example by means of the method of stationary flows. A stationary flow is a time-independent solution of a partial differential equation of the KdV hierarchy. Since the Hamiltonian functionals $\mathcal{H}_{i}$ commute, the set of singular points of each linear combination of the equations of the hierarchy is a finite-dimensional invariant submanifold of the KdV flow. Therefore it makes sense to look for a class of solutions of the KdV equation on a submanifold defined by the stationary flow of an equation of the KdV hierarchy. We consider for example the submanifold $M_{5}$ of the 5 th order jet manifold, defined by

$$
u_{x x x x x}-10 u u_{x x x}-20 u_{x} u_{x x}+30 u^{2} u_{x}=0 .
$$

$M_{5}$ is five dimensional and we can use $\left(u, u_{x}, u_{x x}, u_{x x x}, u_{x x x x}\right)$ as coordinates. So, on the one hand a solution $u(x, t)$ has to satisfy equation (1.3). Using the first equation of the KdV hierarchy this can be written as the following system of ODEs

$$
\begin{aligned}
D_{t_{1}}(u) & =u_{x} \\
D_{t_{1}}\left(u_{x}\right) & =u_{x x} ; \\
D_{t_{1}}\left(u_{x x}\right) & =u_{x x x} ; \\
D_{t_{1}}\left(u_{x x x}\right) & =u_{x x x x} ; \\
D_{t_{1}}\left(u_{x x x x}\right) & =10 u u_{x x x}+20 u_{x} u_{x x}-30 u^{2} u_{x} .
\end{aligned}
$$

In the same way the $\mathrm{KdV}$ equation leads to the system

$$
\begin{aligned}
D_{t_{3}}(u)= & \frac{1}{4}\left(u_{x x x}-6 u u_{x}\right) \\
D_{t_{3}}\left(u_{x}\right)= & \frac{1}{4}\left(u_{x x x x}-6 u u_{x x}-6 u_{x}^{2}\right) \\
D_{t_{3}}\left(u_{x x}\right)= & \frac{1}{4}\left(4 u u_{x x x}+2 u_{x} u_{x x}-30 u^{2} u_{x}\right) ; \\
D_{t_{3}}\left(u_{x x x}\right)= & \frac{1}{4}\left(6 u_{x} u_{x x x}+4 u u_{x x x x}+2 u_{x x}^{2}-60 u u_{x}^{2}-30 u^{2} u_{x x}\right) ; \\
D_{t_{3}}\left(u_{x x x x}\right)= & \frac{1}{4}\left(10 u_{x x} u_{x x x}+10 u_{x} u_{x x x x}+10 u^{2} u_{x x x}\right) \\
& -\frac{1}{4}\left(100 u u_{x} u_{x x}+120 u^{3} u_{x}+60 u_{x}^{3}\right)
\end{aligned}
$$


where we have used constraint (1.3) to eliminate the fifth order derivative. In order to find now the corresponding solution of the $\mathrm{KdV}$ equation in $x$ and $t$, we first have to find a solution $U\left(t_{1}, f\left(t_{3}\right)\right)$ of the first system (1.4) with initial conditions $f\left(t_{3}\right)$. Then we put this solution in system (1.5) and solve it. To conclude we consider the first component $u\left(t_{1}, t_{3}\right)$ and set $t_{1}=x$ and $t_{3}=t$.

What makes this procedure so interesting is that the equations (1.4) and (1.5) have also a bi-Hamiltonian structure (see [34] where the above system is written in function of the first five coefficients $\left.\left(h_{1}, \ldots, h_{5}\right)\right)$. So, the ODEs can be solved by means of the method of separation of variables. This topic will be discussed in section 1.3.

\subsubsection{Recursion operators}

In this subsection, we will elaborate briefly on the concept of recursion operator. For more information we refer to the book of Olver [42].

Consider a fibred manifold $(E, \pi, M)$. The coordinates $x^{i}$ on $M$ denote the independent variables. On $E$ the coordinates will be labelled $\left(x^{i}, u^{\alpha}\right)$ where the $u^{\alpha}$ are the dependent variables. Let $J^{k} \pi$ be the $k$ th order jet manifold of $\pi$ with coordinates $\left(x^{i}, u^{\alpha}, u_{i}^{\alpha}, \ldots, u_{i_{1}, \ldots i_{k}}^{\alpha}\right)$. The function $\pi^{k}: J^{k} \pi \rightarrow M$ is called the source projection. There are canonical projections $\pi_{k, l}: J^{k} \pi \rightarrow J^{l} \pi$, defined for all $k \geq l$. $\pi_{k, 0}: J^{k} \pi \rightarrow E$ is the target projection. For a function $f: J^{k} \pi \rightarrow \mathbb{R}$ (called a differential function), we write $f[u]=$ $f\left(x, u, u_{i}, \ldots, u_{i_{1}, \ldots, i_{k}}\right)$. $\mathcal{A}$ denotes the space of differential functions $f[u]$. Further details about jet bundles can be found in [43].

Consider the following commutative diagram

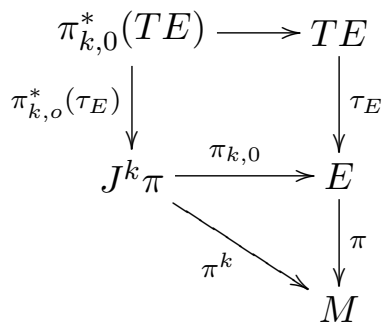

A generalised vector field $\mathbf{v}$ is a section of the pull-back $\pi_{k, 0}^{*}\left(\tau_{E}\right)$ and has coordinate expression

$$
\mathbf{v}=\sum_{i=1}^{p} \xi^{i}[u] \frac{\partial}{\partial x^{i}}+\sum_{\alpha=1}^{q} \phi_{\alpha}[u] \frac{\partial}{\partial u^{\alpha}} .
$$


Associated to $\mathbf{v}$ we can define an evolutionary vector field or vertical representative

$$
\mathbf{v}_{Q}=\sum_{\alpha=1}^{q} Q_{\alpha}[u] \frac{\partial}{\partial u^{\alpha}}
$$

with

$$
Q_{\alpha}[u]=\phi_{\alpha}[u]-\sum_{i=1}^{p} \xi^{i}[u] \frac{\partial u^{\alpha}}{\partial x^{i}} .
$$

The $q$-tuple $Q[u]=\left(Q_{1}[u], \ldots, Q_{q}[u]\right) \in \mathcal{A}^{q}$ is referred to as the characteristic of $\mathbf{v}$. Olver defines a generalised vector field $\mathbf{v}$ to be a generalised infinitesimal symmetry of a system of differential equations $\Delta[u]=$ $\left(\Delta_{1}[u], \ldots, \Delta_{l}[u]\right)=0$ if and only if

$$
\operatorname{prv}\left[\Delta_{v}\right]=0 \quad v=1, \ldots, l
$$

for every solution $u$ of $\Delta[u]=0$. Here prv refers to the (infinite) prolongation of $\mathbf{v}$ given by

$$
\operatorname{prv}=\sum_{i=1}^{p} \xi^{i} \frac{\partial}{\partial x^{i}}+\sum_{\alpha=1}^{q} \sum_{J} \phi_{\alpha}^{J} \frac{\partial}{\partial u_{J}^{\alpha}}
$$

where $\phi_{\alpha}^{J}=D_{J}\left(\phi_{\alpha}-\sum_{i=1}^{p} \xi^{i} u_{i}^{\alpha}\right)+\sum_{i=1}^{p} \xi^{i} u_{J, i}^{\alpha}$. Remark that $\mathbf{v}$ is a symmetry of $\Delta$ if and only if $\mathbf{v}_{Q}$ is. For example, $\mathbf{v}=-\frac{\partial}{\partial x}$ is an infinitesimal symmetry of the $\mathrm{KdV}$ equation ( $\left.\operatorname{prv}=-\frac{\partial}{\partial x}\right)$. The corresponding characteristic is $Q=u_{x}$, so the evolutionary vector field is $\mathbf{v}_{Q}=u_{x} \frac{\partial}{\partial u}$ which is also a symmetry $\left(\operatorname{prv}_{Q}=u_{x} \frac{\partial}{\partial u}+u_{x x} \frac{\partial}{\partial u_{x}}+u_{x t} \frac{\partial}{\partial u_{t}}+u_{x x x} \frac{\partial}{\partial u_{x x}}+\ldots\right)$.

Now we can give the general definition of a recursion operator

Definition 6. A recursion operator for a system of differential equations $\Delta$ is a linear operator $R: \mathcal{A}^{q} \rightarrow \mathcal{A}^{q}$ with the property that whenever $\mathbf{v}_{Q}$ is a symmetry, so is $\mathbf{v}_{\widetilde{Q}}$ with $\widetilde{Q}=R Q$.

So, the recursion operator gives a method for generating an infinite family of symmetries from one given symmetry. Unfortunately, the deduction of the form of the recursion operator (if it exists) requires guesswork, inspired by already known symmetries. The following theorem gives an important characterisation of recursion operators. In this theorem we need the Fréchet derivative $D_{\Delta}$ of a system of differential functions $\Delta$. It is defined by

$$
D_{\Delta}(Q)=\left.\frac{d}{d \varepsilon}\right|_{\varepsilon=0} \Delta[u+\varepsilon Q[u]]
$$


Theorem 1. If $R: \mathcal{A}^{q} \rightarrow \mathcal{A}^{q}$ is a linear operator such that

$$
D_{\Delta} R=\widetilde{R} D_{\Delta}
$$

for all solutions $u$ to $\Delta$, where $\widetilde{R}: \mathcal{A}^{q} \rightarrow \mathcal{A}^{q}$ is a linear differential operator, then $R$ is a recursion operator for the system.

Example: for the KdV equation,

$$
R=D_{x}^{2}-4 u-2 u_{x} D_{x}^{-1}
$$

is a recursion operator. Indeed, it is easy to prove that $R$ satisfies (1.6) with $\widetilde{R}=R$ and the Fréchet derivative calculated as follows:

$$
\begin{aligned}
& D_{\Delta}(Q) \\
& =\left.\frac{d}{d \varepsilon}\right|_{\varepsilon=0}\left[\left(u_{t}+\varepsilon D_{t} Q\right)-\frac{1}{4}\left(u_{x x x}+\varepsilon D_{x}^{3} Q\right)+\frac{3}{2}(u+\varepsilon Q)\left(u_{x}+\varepsilon D_{x} Q\right)\right] \\
& =\left.\left(D_{t} Q-\frac{1}{4} D_{x}^{3} Q+\frac{3}{2} u D_{x} Q+\frac{3}{2} u_{x} Q+3 \varepsilon Q D_{x} Q\right)\right|_{\varepsilon=0} .
\end{aligned}
$$

So, $D_{\Delta}=D_{t}-\frac{1}{4} D_{x}^{3}+\frac{3}{2} u D_{x}+\frac{3}{2} u_{x}$. Because of the integral operator $D_{x}^{-1}$, we have that if $\mathbf{v}_{Q}$ is a symmetry, the $R Q$ will only be defined if $Q=D_{x} A$ for some $A \in \mathcal{A}$. If we apply $R$ to the symmetry $-\frac{\partial}{\partial x}$, we obtain $Q_{2}=R u_{x}=$ $u_{x x x}-6 u u_{x}$, which is equivalent to the characteristic $u_{t}$ of the symmetry $-\frac{\partial}{\partial t}$.

Most of the well known integrable evolution equations do have a recursion operator. In the case of a bi-Hamiltonian system $u_{t}=\mathcal{P}_{0} \frac{\delta}{\delta u} \mathcal{K}_{0}=\mathcal{P}_{1} \frac{\delta}{\delta u} \mathcal{K}_{1}$, one can often find a recursion operator by inverting one of the Poisson operators $R=\mathcal{P}_{1} \mathcal{P}_{0}^{-1}$. We have seen, in the discussion of the $\mathrm{KdV}$ equation, that there exists then a sequence of functionals $\mathcal{H}_{i}$ which provide a collection of conservation laws. In the case of the Toda lattice both Poisson operators are degenerate and therefore the method fails.

To end this subsection, we want to remark that the term recursion operator is also used in the context of ordinary differential equations. Although the purpose of recursion operators (mapping symmetries into symmetries) may be similar, the theory for PDEs on the one hand and for systems of ODEs on the other, have a different methodology. In the context of PDEs, recursion operators map generalised symmetries into new symmetries possibly depending on higher-order derivatives. Therefore a recursion operator will generally be a differential operator of non-zero order. But in the case 
of systems of second order ODEs, the recursion operators are tensors, that is, differential operators of order zero. That's because in this case one can without loss of generality restrict one's attention to symmetries which are vector fields living on the tangent bundle whose flows leave the vector field associated to the second order equations, invariant. The dependence on derivatives of second or higher order can always be eliminated through the given differential equations.

In what follows, we will discuss the term recursion operator in the context of second order differential equations. There are mainly two situations in which a type $(1,1)$ tensor field $R$ is generally referred to as having recursion properties. A first one is the case that $R$ is invariant under the flow of some given dynamics. then $R$ obviously has the property of mapping symmetries into symmetries. The other situation of interest is when the Nijenhuis torsion of $R$

$N_{R}(X, Y)=[R X, R Y]-R[R X, Y]-R[X, R Y]+R^{2}[X, Y] \quad X, Y \in \mathcal{X}(M)$

vanishes. That will be the case, for example, when the manifold under consideration has a Poisson structure and $R$ is the recursion tensor which is responsible for the generation of a bi-Hamiltonian structure, i.e. a PoissonNijenhuis structure. This situation will be discussed in the next subsection.

\subsubsection{Poisson-Nijenhuis structures}

Definition 7. A Poisson-Nijenhuis manifold is a triple $\left(M, P_{0}, R\right)$ such that

- $\left(M, P_{0}\right)$ is a Poisson manifold;

- $R$ is a type $(1,1)$ tensor field on $M$ that satisfies $P_{0} R=R P_{0}^{\dagger}$;

- $P_{1}=R P_{0}$ defines also a Poisson bracket which is compatible with the one associated with $P_{0}$.

So, in this case $\left(M, P_{0}, R\right)$ is a bi-Hamiltonian manifold and $R$ is called the recursion operator. Sometimes $R$ is called the Nijenhuis operator because the compatibility of $P_{0}$ and $P_{1}$ makes the Nijenhuis torsion of $R$ zero.

As a consequence of the assumption that $P_{0} R=R P_{0}, \mu_{R, P_{0}}$ defined by

$$
\mu_{R, P_{0}}(\alpha, X)=\left(\mathcal{L}_{P_{0}(\alpha)} R\right)(X)-P_{0}\left(\mathcal{L}_{X}(R \alpha)\right)+P_{0}\left(\mathcal{L}_{R(X)} \alpha\right),
$$

\footnotetext{
${ }^{\dagger}$ Throughout this thesis we will make no notational distinction between the action of a type $(1,1)$ tensor field and its dual action on 1-forms.
} 
with $\alpha \in \mathcal{X}^{*}(M)$ and $X \in \mathcal{X}(M)$, is a type $(1,2)$ tensor field (see [24]). It is sometimes called the Magri-Morosi concomitant. The necessary and sufficient conditions for $P_{1}=R P_{0}$ to define a compatible Poisson bracket are now:

- the Nijenhuis torsion $N_{R}$ is zero;

- the Magri-Morosi concomitant of $R$ and $P_{0}$ vanishes.

Now we look at the special case that $P_{0}$ comes from a symplectic form and briefly describe the results of [8]. So, suppose that we have a $2 n$ dimensional symplectic manifold $(M, \omega)$. The Poisson bracket associated with $\omega$ is denoted as $\{,\}_{0}$ and the corresponding Poisson map $P_{0}$ is of course invertible. Assume now that we have a type $(1,1)$ tensor $R$ on $M$ satisfying $\omega(R(),)=.\omega(., R()$.$) . An interesting class of such tensors arise$ from the construction of a second 2-form $\omega_{1}$ and the determining formula:

$$
i_{R(\xi)} \omega=i_{\xi} \omega_{1} \quad \forall \xi \in \mathcal{X}(M) .
$$

They have automatically the required symmetry. We call such tensors $R$ tensors. In that case we find an interesting equivalent way of expressing that $\mu_{R, P_{0}}=0$, namely

$$
d \omega_{1}=0 \quad \text { where } \quad \omega_{1}=\frac{1}{2} i_{R} \omega
$$

Another equivalent condition is given by

$$
i_{\mathcal{L}_{X_{f} R}} \omega=-2 d d_{R} f \quad \text { for all functions } f
$$

where $d_{R}:=\left[i_{R}, d\right]=i_{R} d-d i_{R}$.

It is useful to recall here some properties of the derivations $i_{*}$ and $d_{*}$, in the sense of the Frölicher-Nijenhuis theory. Firstly, we have for two arbitrary vector-valued forms $L$ and $M$ of degree $l$ and $m$ respectively, that the graded commutator of $d_{L}$ and $d_{M}$ defines the Nijenhuis bracket of $L$ and $M$ :

$$
\left[d_{L}, d_{M}\right]:=d_{L} d_{M}-(-1)^{l m} d_{M} d_{L}=d_{[L, M]}
$$

and the relation with the Nijenhuis torsion of a type $(1,1)$ tensor field $W$ is that

$$
[W, W]=2 N_{W} .
$$


Further, the general commutator of $i_{L}$ and $d_{M}$ satisfies

$$
\left[i_{L}, d_{M}\right]:=i_{L} d_{M}-(-1)^{(l-1) m} d_{M} i_{L}=d_{i_{L} M}+(-1)^{m} i_{[L, M]} .
$$

Applied to the special case that $L$ and $M$ are both a $(1,1)$ tensor $W$, this becomes

$$
\left[i_{W}, d_{W}\right]=d_{W^{2}}-i_{[W, W]} .
$$

For an operator $d_{W}$ also holds the following lemma.

Definition 8. The operator $d_{W}$, with $W$ a type $(1,1)$ tensor field, is characterised by the following properties:

1. It is a derivation of degree 1 , i.e $d_{W} \alpha$ is a $(k+1)$-form and

$$
d_{W}(\alpha \wedge \beta)=\left(d_{W} \alpha\right) \wedge \beta+(-1)^{k} \alpha \wedge\left(d_{W} \beta\right)
$$

if $\alpha$ is a $k$-form.

2. The graded commutator of $d_{W}$ and the exterior derivative $d$ vanishes:

$$
\left[d_{W}, d\right]=d_{W} d+d d_{W}=0 .
$$

3. Its action on $C^{\infty}(M)$ is given by:

$$
d_{W} f=W(d f) .
$$

The necessary and sufficient condition for $d_{W}$ to satisfy $d_{W}^{2}=0$ is that the Nijenhuis torsion of $W$ must be zero. That is the case here for $R$ and therefore $\left(d, d_{R}\right)$ defines a simple bi-differential calculus

Definition 9. A simple bi-differential calculus on $\Lambda(M)$ is a pair $\left(d_{1}, d_{2}\right)$ of derivations of $\Lambda(M)$ of degree 1 which both have the co-boundary property $d_{i}^{2}=0$ and $\left[d_{1}, d_{2}\right]=d_{1} d_{2}+d_{2} d_{1}=0$.

The benefit of such a bi-differential calculus $\left(d_{1}, d_{2}\right)$ is that if $\chi^{(0)} \in C^{\infty}(M)$ satisfies $d_{1} d_{2} \chi^{(0)}=-d_{2} d_{1} \chi^{(0)}=0$, we can inductively define a sequence of functions $\chi^{(i)}$ according to the rule:

$$
d_{1} \chi^{(i+1)}=d_{2} \chi^{(i)} .
$$

So, let now $\chi^{(0)}$ be a function satisfying $d d_{R} \chi^{(0)}=0$, then we have 
Theorem 2. Suppose that $(M, \omega)$ is a symplectic manifold and that $R$ is a type $(1,1)$ tensor symmetric w.r.t $\omega$. If $\left(\Lambda(M), d, d_{R}\right)$ is a bi-differential calculus and $d d_{R} \chi^{(0)}=0$, then the functions $\chi^{(i)}$ defined by $d \chi^{(i+1)}=d_{R} \chi^{(i)}$ are in involution w.r.t the Poisson brackets defined by $P_{0}$ (corresponding to $\omega)$ and $R P_{0}$.

For future use, we recall here also the definition of the more general 'gauged bi-differential calculus' given by Dimakis and Müller-Hoissen (see [14]).

Definition 10. Suppose that we have a bi-differential calculus $\left(\Lambda(M), d_{1}, d_{2}\right)$. $A$ gauged bi-differential calculus on $\Lambda(M)$ is a pair of operators $\left(D_{1}, D_{2}\right)$ of the form $D_{i}=d_{i}+A_{i}$ where the $A_{i}$ are $m \times m$ matrices of 1-forms, which act on $m \times m$ matrices of forms by matrix-wedge multiplication. The $D_{i}$ further have to satisfy the conditions: $D_{i}^{2}=0$ and $\left[D_{1}, D_{2}\right]=D_{1} D_{2}+D_{2} D_{1}=0$.

Actually, we will use a slightly different definition, namely where the operators $D_{i}$ act on $m$-component column vectors of forms in stead of on $m \times m$ matrices.

Coming back now to the problem of complete integrability we can recall the following theorem (see [49]).

Theorem 3. Consider a 2n-dimensional Poisson-Nijenhuis manifold $\left(M, P_{0}, R\right)$. If $R$ has exactly $n$ functionally independent eigenvalues, then the system is completely integrable.

If $R$ has at every point of $M n$ (double) distinct eigenvalues $\lambda_{i}$ then $\left(M, P_{0}, R\right)$ is called semisimple and has a neighbourhood where Darboux-Nijenhuis coordinates can be found. These coordinates play an important role in the study of separability of the Hamilton-Jacobi equation.

Definition 11. A set of local coordinates $(x, y)$ on a $2 n$-dimensional PoissonNijenhuis manifold is called a set of Darboux-Nijenhuis coordinates if they satisfy the following two conditions:

- they are canonical w.r.t $P_{0}$ :

$$
\left\{x_{i}, y_{j}\right\}_{0}=\delta_{i j}, \quad\left\{x_{i}, x_{j}\right\}_{0}=\left\{y_{i}, y_{j}\right\}_{0}=0
$$

- they put the recursion tensor $R$ in diagonal form:

$$
R=\sum_{i=1}^{n} \lambda_{i}\left(\frac{\partial}{\partial x^{i}} \otimes d x^{i}+\frac{\partial}{\partial y^{i}} \otimes d y^{i}\right)
$$


Moreover, as a consequence of the vanishing of the Nijenhuis torsion of $R$, the eigenvalues $\lambda_{i}$ always satisfy

$$
R d \lambda_{i}=\lambda_{i} d \lambda_{i}
$$

From this then follows that the $\lambda_{i}$ only depend on the coordinates $\left(x_{i}, y_{i}\right)$, we say that $R$ is separable in coordinates.

Assume now that $R$ has $n$ distinct eigenvalues $\lambda_{i}$ which are functionally independent. Then there exist $n$ functions $\mu_{i}$ such that $\left(\lambda_{i}, \mu_{i}\right)$ are DarbouxNijenhuis coordinates.

\subsection{Separability of the Hamilton-Jacobi equation}

In this section we summarise a number of historical developments in the area of 'separation of variables'. The problem can be formulated as follows: suppose that we have a Hamiltonian system $H$ of dimension $2 n$. The Hamilton-Jacobi equation is given by

$$
H\left(q, \frac{\partial W}{\partial q}\right)=\alpha_{1} .
$$

It is in general very difficult to solve but in certain cases one can do it using separation of variables. This means that one tries to find a complete solution that has the form

$$
W(q, \alpha)=\sum_{i=1}^{n} W_{i}\left(q^{i}, \alpha\right) .
$$

If such a solution exists, we say that the Hamilton-Jacobi equation admits a separated solution. $H$ is then said to be separable and the $W^{i}\left(q^{i}, \alpha\right)$ can in principle be determined by quadratures.

Suppose now that $H$ is integrable, i.e. along with $H=I_{1}$ we have further $n-1$ functionally independent, pairwise commuting integrals of motion $I_{2}, \ldots, I_{n}$. An integrable system $\left(I_{1}, \ldots, I_{n}\right)$ is separable if the HamiltonJacobi equation associated with any $I_{k}$ is separable. In that case we have an equivalent definition (see for example [46]).

Definition 12. An integrable system $\left(I_{1}, \ldots, I_{n}\right)$ is separable in the coordinates $(q, p)$ if their exist $n$ non-trivial relations

$$
\Phi_{i}\left(q^{i}, p_{i} ; I_{1}, \ldots, I_{n}\right)=0 \quad i=1, \ldots, n .
$$

Furthermore, if the coordinates $q$ are orthogonal with respect to a given metric, the system is said to be orthogonally separable. 


\subsubsection{Liouville systems}

Liouville was the first to study separability of the Hamilton-Jacobi equation. In 1849 [29] he proved that the complete integral of the so-called Liouville systems which are systems with a Hamiltonian of the form

$$
H=T+V
$$

where

$$
\begin{aligned}
T & =\frac{c}{2} \sum_{i=1}^{n} \frac{\left(\dot{q}^{i}\right)^{2}}{a_{i}\left(q^{i}\right)}=\frac{1}{2 c} \sum_{i=1}^{n} a_{i}\left(q^{i}\right) p_{i}^{2} \\
V & =\frac{1}{c} \sum_{i=1}^{n} V_{i}\left(q^{i}\right) \\
c & =\sum_{i=1}^{n} c_{i}\left(q^{i}\right) .
\end{aligned}
$$

can be determined by the method of separation of variables. The set of first integrals is given by

$$
H \quad \text { and } \quad I_{i}=\frac{1}{2} a_{i} p_{i}^{2}+V_{i}-c_{i} H \quad \text { for } \quad i=1, \ldots, n-1 .
$$

\subsubsection{Stäckel systems}

The famous Stäckel theorem tells how separable systems look like in (orthogonal) coordinates for which the separation is possible.

Stäckel's first major contribution was to find all separable metrics for an arbitrary two dimensional Riemannian manifold. They can all be written locally in Liouville form ([41]). For dimension greater than 2, there are also separable systems of an other form, for example the ones with Hamiltonians of the form:

$$
H=\sum_{i=1}^{n} c_{i}(q)\left[\frac{1}{2} p_{i}^{2}+V_{i}\left(q^{i}\right)\right] .
$$

Stäckel proved in 1891 (see [47]) the following theorem which connects the separability of such systems with the existence of a matrix $S$, now called a Stäckel matrix. 
Theorem 4. A dynamical system with a Hamiltonian of the form (1.14) is separable if and only if there exists an invertible $n \times n$ matrix $S=\left(S_{i j}\right)$ with $S_{i j}$ function of $q^{i}$ alone such that

$$
\sum_{i=1}^{n} c_{i} S_{i k}=\delta_{k}^{1} \quad k=1, \ldots, n
$$

In that case a Hamiltonian system of the form (1.14) is called a Stäckel (separable) system. Remark that from (1.15) follows that $\left[c_{1}, \ldots, c_{n}\right]$ is the first row of $S^{-1}=\left(a_{i j}\right)$.

The $n$ independent constants of motion in involution for this system are constructed as

$$
I_{j}=\sum_{i=1}^{n} a_{i j}\left[\frac{1}{2} p_{i}^{2}+V_{i}\left(q^{i}\right)\right] \quad j=1, \ldots, n
$$

where $I_{1}=H$.

Definition 13. Let $\left(I_{1}, \ldots, I_{n}\right)$ be an integrable system. It is called Stäckel separable if the relations (1.13) are affine, i.e. they have the following form:

$$
\sum_{i=1}^{N} S_{i k} I_{k}-U_{i}=0
$$

with $S$ an invertible matrix.

\subsubsection{Levi-Civita conditions}

In 1904 Levi-Civita found a test for the separability of a Hamiltonian system $H(q, p)$.

Theorem 5. The Hamilton-Jacobi equation separates in the coordinates $\left(q^{i}, p_{i}\right)$ if and only if the Hamiltonian satisfies

$$
\frac{\partial H}{\partial p_{i}} \frac{\partial H}{\partial p_{j}} \frac{\partial^{2} H}{\partial q^{i} \partial q^{j}}-\frac{\partial H}{\partial p_{i}} \frac{\partial H}{\partial q^{j}} \frac{\partial^{2} H}{\partial q^{i} \partial p_{j}}-\frac{\partial H}{\partial q^{i}} \frac{\partial H}{\partial p_{j}} \frac{\partial^{2} H}{\partial p_{i} \partial q^{j}}+\frac{\partial H}{\partial q^{i}} \frac{\partial H}{\partial q^{j}} \frac{\partial^{2} H}{\partial p_{i} \partial p_{j}}=0,
$$

$i, j=1, \ldots, n$ and $i \neq j$.(There is no summation over the indices!) 
Levi-Civita showed also that for a natural Hamiltonian, i.e. a Hamiltonian of type $T+V$, to be separable, it is necessary that the kinetic part by itself is separable. Once the separation variables have been detected, there are simple criteria to see if the potential term is compatible with the separation. So, when $H=\frac{1}{2} \sum_{i=1}^{n} g^{i i}(q) p_{i}^{2}+V(q)$ (orthogonal coordinates), the conditions (1.16) become

$$
\frac{\partial^{2} g^{k k}}{\partial q^{i} \partial q^{j}}-\frac{\partial \ln g^{j j}}{\partial q^{i}} \frac{\partial g^{k k}}{\partial q^{j}}-\frac{\partial \ln g^{i i}}{\partial q^{j}} \frac{\partial g^{k k}}{\partial q^{i}}=0
$$

and

$$
\frac{\partial^{2} V}{\partial q^{i} \partial q^{j}}-\frac{\partial \ln g^{j j}}{\partial q^{i}} \frac{\partial V}{\partial q^{j}}-\frac{\partial \ln g^{i i}}{\partial q^{j}} \frac{\partial V}{\partial q^{i}}=0
$$

Levi-Civita's theorem is a very important result but if suffers from a major disadvantage, namely it doesn't tell how to construct the separation coordinates or if they even exist. The first theorem that gives an answer to that problem is given by Eisenhart (see 1.3.4).

Another intrinsic way to write down the Levi-Civita conditions was found by Magri and inspired Crampin [12] to state the following proposition: if the Hamilton-Jacobi equation for $h=\frac{1}{2} \sum_{i} g^{i i} p_{i}^{2}$ is separable in coordinates $\left(q^{i}\right)$, then for every symmetric type $(1,1)$ tensor field $J$ associated with the diagonal equivalence class of coordinates $\left(q^{i}\right)$,

$$
d d_{\widetilde{J}} h=\sum_{r=0}^{n-1} \alpha^{(r)} \wedge d_{\widetilde{J}^{r}} h
$$

with $\widetilde{J}$ the complete lift of $J$ and $\alpha^{(r)}$ 1-forms. With ' $J$ associated to a diagonal equivalence class of coordinates $\left(\left(q_{1}^{i}\right) \sim\left(q_{2}^{i}\right) \text { if } q_{1}^{i}=q_{1}^{i}\left(q_{2}^{i}\right)\right)^{\prime}$, we mean that (a) $J$ is diagonal w.r.t the coordinates of the class, (b) at each point the eigenvalues of $J$ are simple, (c) the Nijenhuis torsion of $J$ vanishes.

Conversely, if there is a type $(1,1)$ tensor field $J$ which is symmetric w.r.t $g$, has pointwise simple eigenvalues and vanishing Nijenhuis torsion and if there exist 1-forms $\alpha^{(r)}$ such that $\widetilde{J}$ satisfies (1.19), then any coordinates w.r.t which $J$ is diagonal are orthogonal separation coordinates for $h$.

\subsubsection{Eisenhart's theorem}

In 1934 Eisenhart investigated the existence of orthogonal separation variables on Riemannian manifolds [15]. His result is given in a coordinate free 
form by considering special families of Killing tensors of order 2. A Killing tensor $K$ of order 2 is a $(0,2)$ type symmetric tensor such that $K_{(i j \mid l)}=0$. For more information about Killing tensors see section 2.1.

Theorem 6. The geodesic Hamiltonian $H=\frac{1}{2} \sum_{i, j=1}^{n} g^{i j}(q) p_{i} p_{j}$ can be transformed into the Stäckel form (and therefore is orthogonally separable) if and only if the following conditions are satisfied:

1. there exist $n$ linearly independent Killing tensors $K_{(m)}$ for $m=1, \ldots, n$ with $K_{(1)}=g$;

2. the quadratic functions $H_{(m)}=\frac{1}{2} \sum_{i, j=1}^{n} K_{(m)}^{i j} p_{i} p_{j}$ commute pairwise with respect to the standard Poisson bracket;

3. each $K_{(m)}(m>1)$ has n functionally independent eigenfunctions $\lambda_{(m)}^{(k)}$ and the $n \times n$ matrix whose rows are the eigenfunctions of $K_{(m)}$ $(m=1, \ldots, n)$ is everywhere non-singular;

4. $K_{(m)}$ are simultaneously diagonalisable and one can find a set of simultaneous eigenforms $\alpha^{(k)}$ for them which are closed:

$$
\sum_{i=1}^{n}\left(K_{(m)}^{i j}-\lambda_{(m)}^{(k)} g^{i j}\right) \alpha_{i}^{(k)}=0 \quad \text { with } \quad d \alpha^{(k)}=0 .
$$

The closed eigenforms determine an orthogonal coordinate system $\left\{a_{k}\right\}$ (when $\alpha^{(k)}=d a_{k}$ ) with respect to which the tensors $K_{(m)}$ are diagonal. The fact that $K_{(m)}$ are simultaneously diagonalised in orthogonal coordinates is equivalent with saying that there are $n$ common normal eigenvectors. This means that the orthogonal distribution of such an eigenvector is Fröbenius integrable.

The Levi-Civita conditions for orthogonal coordinates (1.17) are just the integrability conditions for the tensors $K_{(m)}$ to be Killing tensors.

In what follows, we call a set of tensors $K_{(m)}$ satisfying the conditions of Eisenhart's theorem a Killing-Stäckel system (following Benenti [3]).

\subsubsection{Benenti systems}

In [2] Benenti simplified Eisenhart's theorem by stating that any Stäckel system can be represented by just one of its Killing tensors. 
Theorem 7. A geodesic Hamiltonian is separable in orthogonal coordinates if and only if on the Riemann manifold there exists a Killing tensor with pointwise simple real eigenvalues, normal eigenvectors (or closed eigenforms). Such a tensor is called a characteristic Killing tensor.

For a natural Hamiltonian $H=T+V$ the Hamilton-Jacobi equation admits a separated solution in orthogonal coordinates if and only if there exists a characteristic Killing tensor $\hat{K}$ and the potential satisfies

$$
d(\hat{K} d V)=0 .
$$

Among all Stäckel systems a particularly important subclass consists of those constructed with the help of a so-called special conformal Killing tensor $L$. In 1992 Benenti has obtained a simple recurrence procedure to construct a family of Killing tensors obeying Eisenhart's conditions, obtained from the tensor $L[1]$. This kind of systems are now called Benenti systems. We will discuss this in detail in the next chapter.

\subsubsection{Separability for bi-Hamiltonian systems}

Suppose that $\left(M, P_{0}, R\right)$ is a $2 n$-dimensional semisimple Poisson-Nijenhuis manifold with eigenvalues $\lambda_{1}, \ldots, \lambda_{n}$ as defined in paragraph 1.2.2 . Let $\left(I_{1}, \ldots I_{n}\right)$ be a set of functionally independent functions. Then the following statements are equivalent

1. $\left(I_{1}, \ldots, I_{n}\right)$ is separable in Darboux-Nijenhuis coordinates.

2. The functions $I_{i}$ are in involution with respect to both Poisson brackets: $\left\{I_{i}, I_{j}\right\}_{0}=0=\left\{I_{i}, I_{j}\right\}_{1}$.

3. There exists a simple matrix $F$ such that

$$
R d I_{i}=\sum_{j=1}^{n} F_{i j} d I_{j}
$$

or the subspace spanned by $\left(d I_{1}, \ldots d I_{n}\right)$ is invariant w.r.t $R$.

The matrix $F$ is called the control matrix and its eigenvalues are the same as those of $R$. If we look at the stronger condition of Stäckel separability, 
we find that $\left(I_{1}, \ldots, I_{n}\right)$ are Stäckel separable if and only if in addition to condition (1.20), the control matrix $F$ satisfies

$$
R d F_{i j}=\sum_{j=1}^{n} F_{i k} d F_{k j} .
$$

The relation between $F$ and the Stäckel matrix $S$ is given by

$$
F_{i j}=\sum_{k=1}^{n} S_{i k}^{-1} \lambda_{k} S_{k j} .
$$

Proofs of these statements can be found in [17]. 


\section{Chapter 2}

\section{Special conformal Killing tensors}

\subsection{Killing tensors}

We consider a pseudo-Riemannian manifold $(Q, g)$ of dimension $n .\{\}$ is the standard Poisson bracket on the cotangent bundle $T^{*} Q$ with Poisson bivector $\Pi=\frac{\partial}{\partial p_{i}} \wedge \frac{\partial}{\partial q^{i}}$. The function $H=\frac{1}{2} g^{i j} p_{i} p_{j}^{\ddagger}$ is the geodesic Hamiltonian. If $W=\left(W^{i \ldots j}\right)$ is a contravariant symmetric tensor on $Q$, then we denote by $\mathrm{P}(W)$ the polynomial function on $T^{*} Q$ defined by

$$
\mathrm{P}(W)=W^{i \ldots j} p_{i} \ldots p_{j} .
$$

Definition 14. A vector field $X=X^{k} \frac{\partial}{\partial q^{k}}$ on $Q$ is a Killing vector if

$$
\mathcal{L}_{X} g=0
$$

or equivalently

$$
\{\mathrm{P}(X), H\}=0 \text {. }
$$

In coordinates this means that

$$
X_{i \mid j}+X_{j \mid i}=0
$$

where $X_{i}=g_{i k} X^{k}$. This concept can be generalised to Killing tensors as follows:

Definition 15. A Killing tensor of rank 2 is a symmetric type (2,0) tensor $K$ on $Q$ satisfying

$$
\{\mathrm{P}(K), H\}=0 .
$$

\footnotetext{
${ }^{\ddagger}$ Here and in the rest of the thesis, the Einstein summation convention is used, unless otherwise said.
} 
So, $\mathcal{P}(K)$ is a first integral of $H$. When we include also a potential term, i.e. we consider the natural Hamiltonian $\frac{1}{2} g^{i j} p_{i} p_{j}+V(q)$ then $K^{i j} p_{i} p_{j}$ is a first integral if and only if $K$ is a Killing tensor and

$$
K_{s}^{l} \frac{\partial V}{\partial q^{s}}=0 \quad \text { or } \quad K d V=0 .
$$

In coordinates condition (2.1) is given by

$$
K_{(i j \mid k)}=0
$$

where the brackets denote the cyclic sum over the indices $i, j$ and $k$. The type $(0,2)$ tensor $g_{i k} K^{k l} g_{l j}=K_{i j}$ is also called a Killing tensor. In 1896 Levi-Civita introduced a special kind of Killing tensors, called A-tensors (see Benenti [3]), defined by

$$
A_{i j \mid k}=\mu_{h} A_{i j}-\frac{1}{2}\left(\mu_{j} A_{h i}+\mu_{i} A_{h j}\right) \quad \text { for some } \mu .
$$

These tensors will be important in relation to special conformal Killing tensors (see section 2.3).

\subsection{Conformal Killing tensors}

Definition 16. A symmetric type (2,0) tensor $K=\left(K^{i j}\right)$ on a Riemannian manifold $Q$ is a conformal Killing tensor if

$$
\{\mathrm{P}(K), H\}=-2 \mathrm{P}(A) H
$$

for some vector field $A$ on $Q$.

An equivalent definition is

$$
K_{(i j \mid k)}=\alpha_{(k} g_{i j)}
$$

where $\alpha$ is a 1-form given by $g(A, X)=-\alpha(X)$ for every vector field $X$ on $Q$. If $\alpha=0$ then $K$ is a Killing tensor. A conformal Killing tensor is said to be of gradient type if $\alpha=d f$ or of trace type if $\alpha=d(\operatorname{tr} K)$. The $\operatorname{trace} \operatorname{tr} K$ is defined as $\operatorname{tr}\left(K_{j}^{i}\right)$ and $\operatorname{det} K:=\operatorname{det}\left(K_{j}^{i}\right)$. 


\subsection{Special conformal Killing tensors}

The special conformal Killing tensors which we will discuss in this section are also conformal Killing tensors. The name was introduced by Crampin, Sarlet and Thompson $[8,9,10]$ but tensors of this type were already used in 1992 by Benenti in the case that they have pointwise real simple eigenvalues [1]. In that case they are now often called Benenti tensors . On Euclidean space they were studied by Benenti (also in [1]) and Lundmark [30] and were then also called, respectively, 'planar inertia tensors' and 'elliptic coordinates matrices'.

Definition 17. A symmetric type (0,2) tensor $L$ on a Riemann manifold $Q$ such that

$$
L_{i j \mid k}=\frac{1}{2}\left(\alpha_{i} g_{j k}+\alpha_{j} g_{i k}\right) .
$$

for some $\alpha_{i}$, is called a special conformal Killing tensor (SCK tensor).

Note that any Riemannian manifold $(Q, g)$ admits a trivial special conformal Killing tensor, namely the metric $g$. The equivalent condition for the $(1,1)$ form of the tensor $L$ is

$$
L_{j \mid k}^{i}=\frac{1}{2}\left(\alpha_{j} \delta_{k}^{i}+\alpha^{i} g_{j k}\right) .
$$

Contracting $i$ and $j$, one sees that $\alpha=d(\operatorname{tr} L)$. Another way to write (2.3) is

$$
\{\mathrm{P}(L), H\}=-2 \mathrm{P}\left(\frac{\partial \operatorname{tr} L}{\partial q^{j}} g^{i j}\right) H
$$

So, $L$ is a conformal Killing tensor of trace type. A special conformal Killing tensor has a lot of interesting properties (for the proofs we refer for example to [11]). The most significant one is about its Nijenhuis torsion.

Proposition 1. If $L$ is a special conformal Killing tensor, then its Nijenhuis tensor $N_{L}$ vanishes.

For the converse we have (see theorem 19.3 in [3]):

Proposition 2. A torsionless conformal Killing tensor of trace type is a special conformal Killing tensor.

The fact that $N_{L}=0$ has some important consequences. We can use for example the following interesting property for a general torsionless tensor: 
Lemma 1. For any torsionless type $(1,1)$ tensor $S$ :

$$
S d(\operatorname{det} S)=(\operatorname{det} S) d(\operatorname{tr} S) .
$$

If $L=\left(L_{j}^{i}\right)$ is a non-singular special conformal Killing tensor, we can consider its cofactor tensor $A$ defined as $A=L^{-1} \operatorname{det} L$ and then it can be proven, using lemma 1 , that

Proposition 3. If $L$ is a non-singular special conformal Killing tensor, then its cofactor $A$ is a Killing tensor.

Moreover, the cofactor $A$ is a A-tensor (see $(2.2)$ ) with $\mu_{i}=\left(L^{-1}\right)_{j}^{i} \alpha_{j}=$ $\partial_{i} \log |\operatorname{det} L|$.

We shall often consider special conformal Killing tensors $L$ with $n$ functionally independent eigenvalues $\lambda^{i}$. Then $\left\{\lambda^{i}\right\}$ can be taken as local coordinates. Because the Nijenhuis tensor of $L$ vanishes, $L$ takes the form:

$$
L=\sum_{i=1}^{n} \lambda^{i} \frac{\partial}{\partial \lambda^{i}} \otimes d \lambda^{i}
$$

with respect to them. $L$ is symmetric, so the coordinates are orthogonal i.e. $g_{i j}=0$ for $i \neq j$. That this is a natural assumption follows for example from:

Proposition 4. A conformal Killing tensor with functionally independent eigenfunctions whose Nijenhuis torsion vanishes is a special conformal Killing tensor.

\subsection{SCK tensors and separability}

As said before, in subsection 1.2.5, special conformal Killing tensors play an important role in the study of separability:

Theorem 8. If a Riemannian metric $g$ admits a special conformal Killing tensor with pointwise simple eigenvalues, then the Hamilton-Jacobi equation for geodesics is separable. Moreover if the eigenfunctions are functionally independent they are orthogonal separation coordinates.

For a proof see [10]. Now we will show how a special conformal Killing tensor determines a Killing-Stäckel system (see subsection 1.3.4). But remember 
that not every Killing-Stäckel system is generated by a special conformal Killing tensor.

When the Riemannian metric $g$ admits a special conformal Killing tensor $L$ with (real) simple eigenvalues, then the tensor $\hat{K}=(\operatorname{tr} L) g-L$ is a characteristic Killing tensor (see theorem 7) and

Theorem 9. [3] The set of tensors $\left\{K_{(m)}\right\}=\left\{K_{(1)}, \ldots, K_{(n)}\right\}$ defined by

$$
K_{(1)}=g, \quad K_{(m+1)}=\frac{1}{m} \operatorname{tr}\left(K_{(m)} L\right) g-K_{(m)} L
$$

is a Killing-Stäckel system if and only if $L$ is a special conformal Killing tensor with simple eigenvectors.

Remark that $\hat{K}=K_{(2)}$. An equivalent definition of the sequence (2.5) is given by

$$
K_{(m+1)}=(-1)^{m} \rho_{m} g-K_{(m)} L \quad \text { with } \quad K_{(1)}=g
$$

with $\rho_{m}$ the coefficients of the characteristic polynomial of $L, \operatorname{det}(\lambda I-L)=$ $\lambda^{n}+\rho_{1} \lambda^{n-1}+\ldots+\rho_{n-1} \lambda+\rho_{n}(\operatorname{see}[4])$. When we know the eigenvalues $\lambda^{i}$ of $L$, we can also write

$$
K_{(m+1)}=\sigma_{m} g-K_{(m)} L \quad \text { with } \quad K_{(1)}=g
$$

where $\sigma_{m}$ is the elementary symmetric polynomial of order $m$ of the eigenvalues $\lambda^{i}$. The fact that this last formula requires the knowledge of the eigenvalues, makes it less effective. There is also a relation between the sequence of Killing tensors (2.5) and the cofactor tensor of $L+k I$ (see the end of section 2.5).

Special conformal Killing tensors arise also in other contexts. That will be the content of the next sections of this chapter.

\subsection{SCK tensors and cofactor systems}

Cofactor systems were introduced in Euclidean space by Lundmark [30] and were generalised to Riemannian manifolds $(Q, g)$ by Crampin and Sarlet [9] (see also [31]).

Suppose that we have a non-conservative Lagrangian system with Lagrangian $\ell$. This is described by the second order differential equation field $\Gamma$ on $T Q$ that satisfies

$$
\Gamma\left(\frac{\partial \ell}{\partial u^{i}}\right)-\frac{\partial \ell}{\partial q^{i}}=-M_{i}
$$


where the non-conservative forces $M_{i}$ are the components of a semi-basic 1-form $\mu=M_{i}(q, u) d q^{i}$. Here, we consider the particular case that $\mu$ is a 1-form on $Q$ (in other words the $M_{i}$ do not depend on the velocities) and $\ell$ is a pure kinetic energy function $\ell=\frac{1}{2} g_{i j}(q) u^{i} u^{j}$.

Consider now a non-singular special conformal Killing tensor $L$. This may be used to define two differential operators. In the first place we can form $d_{L}$ (see definition 8). Because the Nijenhuis torsion $N_{L}$ vanishes, the operator $d_{L}$ satisfies $d_{L}^{2}=0$ and therefore $\left(d, d_{L}\right)$ is a simple bi-differential calculus (see definition 9). Since $L$ is non-singular, $d_{L}$ satisfies also a Poincaré lemma, namely $d_{L} \theta=0$ for a $k$-form $\theta$ if and only if there exists locally a $(k-1)$-form $\phi$ such that $\theta=d_{L} \phi$.

Definition 18. Let $L$ be a non-singular special conformal Killing tensor. The operator $D_{L}$ is defined as follows:

$$
D_{L} \theta=(\operatorname{det} L)^{-1} d_{L}((\operatorname{det} L) \theta) \stackrel{2.4}{=} d_{L} \theta+\alpha \wedge \theta \text { for all forms } \theta \text { on } Q
$$

where $\alpha$ is the 1-form associated with $L$.

Remark that $D_{L}$ is not a derivation. It satisfies $D_{L}^{2}=0$ and has the property: ' $D_{L}$-closed' is equivalent to 'locally $D_{L}$-exact'. $\left(d, D_{L}\right)$ is then a gauged bidifferential calculus (see definition 10) with $m=1$.

Now we are ready to define the so-called cofactor systems.

Definition 19. A non-conservative system $\Gamma$ on $T Q$, generated by a couple $(g, \mu)$ is said to be a cofactor system, if $g$ admits a non-singular special conformal Killing tensor $L$ and $\mu$ satisfies $D_{L} \mu=0$.

In [3], another (locally) equivalent characterisation of a cofactor system is given, namely the non-conservative force $\mu$ has to satisfy

$$
\mu=(\operatorname{cof} L)^{-1} \operatorname{grad} V=(\operatorname{det} L)^{-1} d_{L} V=D_{L}\left((\operatorname{det} L)^{-1} V\right)
$$

for some function $V$, where $L$ is a special conformal Killing tensor.

Cofactor systems can be interpreted in a natural way as non-conservative systems admitting a quasi-Hamiltonian representation. The following result was found by Crampin and Sarlet [9]: suppose that we have a nonconservative system $\Gamma$ defined by $(g, \mu)$ and type $(1,1)$ tensor $L$ with $N_{L}=0$. $L$ defines a Poisson structure $P_{L}=\widetilde{L} \circ P_{0}$ where $\widetilde{L}$ is the complete lift of $L$ to $T^{*} Q$ and $P_{0}$ is the standard Poisson map on $T^{*} Q$. The given system satisfies 
$F \widehat{\Gamma}=P_{L}(d H)$ for some function $F$ and a function $H=\frac{1}{2} A^{i j} p_{i} p_{j}+V(q)$ if (a) $L$ is a special conformal Killing tensor, (b) $F=k \operatorname{det} L$ (one can set $F=\operatorname{det} L$ without loss of generality), (c) $A$ is the cofactor tensor of $L$ and (d) $\mu=D_{L}\left((\operatorname{det} L)^{-1} V\right)$. This result can be summarised as follows:

Theorem 10. A non-conservative system $\Gamma$ on $T Q$ determined by $(g, \mu)$ on $Q$ has a quasi-Hamiltonian representation $F \widehat{\Gamma}=P_{L}(d H)$ where $\widehat{\Gamma}$ is the Legendre transform of $\Gamma, L$ is a type $(1,1)$ tensor field on $Q$ and $H$ is a function on $T^{*} Q$ quadratic in momenta, if and only if it is a cofactor system.

Remark that the force $\mu$ is uniquely determined by $H$ which is a quadratic first integral of cofactor type. This explains the term 'cofactor system'.

In [9] it is further shown that $\widehat{\Gamma}$ can also be represented as the restriction to $z=0$ of a Hamiltonian vector field on $T^{*} Q \times \mathbb{R}$. Namely, one can construct a Poisson bivector $\Pi_{L}$ on $T^{*} Q \times \mathbb{R}$

$$
\widehat{\Pi}_{L}=\Pi_{L}+\left(\widehat{\Gamma}-z \alpha^{V}\right) \wedge \frac{\partial}{\partial z} \quad \text { where } \quad \alpha=d(\operatorname{tr} L)
$$

and then $\widehat{\Gamma}+z V=-\widehat{P_{L}}(d z)$ and $H+z \operatorname{det} L$ is a Casimir of $\widehat{\Pi}_{L}$. (For the general theorem about the construction of a Hamiltonian vector field on the extended space starting from an arbitrary quasi-Hamiltonian vector field see [9].)

The situation becomes however more interesting when we have a system that is of cofactor type in two ways, a cofactor pair system.

Definition 20. A cofactor pair system is a non-conservative system $\Gamma$ defined by $(g, \mu)$ where $\mu$ satisfies $D_{L} \mu=D_{K} \mu=0$ for two non-singular special conformal Killing tensors $L$ and $K$.

It is not surprising that such a system will have a double quasi-Hamiltonian representation, called bi-quasi-Hamiltonian structure and that it admits two independent integrals of motion of cofactor pair type. But there is more: a cofactor pair system is completely integrable.

$L+k K$ (with $k$ a constant) is also a special conformal Killing tensor and further we have that $D_{L+k K} \mu=D_{L} \mu+k D_{K} \mu=0$. So, from theorem 10 follows that

$$
\operatorname{det}(L+k K) \widehat{\Gamma}=P_{L+k K}\left(\frac{1}{2} A_{L+k K}^{i j} p_{i} p_{j}+V_{L+k K}\right)
$$


where $A_{L+k K}$ is the cofactor tensor of $L+k K$ and $V_{L+k K}$ is a solution of

$$
D_{L+k K}\left(\operatorname{det}(L+k K)^{-1} V_{L+k K}\right)=\mu .
$$

Just as before, we have then also that

$$
C=\frac{1}{2} A_{L+k K}^{i j} p_{i} p_{j}+V_{L+k K}+z \operatorname{det}(L+k K)
$$

is a Casimir of the Poisson structure

$$
\widehat{\Pi}=\Pi_{L}+k \Pi_{K}+\left(\widehat{\Gamma}-z\left(\alpha^{V}+k \beta^{V}\right)\right) \wedge \frac{\partial}{\partial z}
$$

on $T^{*} Q \times \mathbb{R}$, where $\alpha=d(\operatorname{tr} L)$ and $\beta=d(\operatorname{tr} K)$. Now if we set

$$
A_{L+k K}=\sum_{m=1}^{n} A_{(m)} k^{m-1}
$$

and

$$
V_{L+k K}=\sum_{m=1}^{n} V_{(m)} k^{m-1}
$$

and exploit the fact that $C$ is a Casimir, we find

Theorem 11. A cofactor pair system admits $n$ integrals of motion

$$
I_{(m)}=\frac{1}{2} A_{(m) i j} v^{i} v^{j}+V_{(m)}(q)
$$

which are in involution with respect to the Poisson brackets associated to $P_{L}$ and $P_{K}$.

To end this section, it is interesting to show the link between these results and the separability theory: if we put $K=I$ and if $L$ has $n$ functionally independent eigenfunctions $\lambda^{i}$, we can generate a Killing-Stäckel system (see subsection 1.3.4 and section 2.4). Indeed, we know that the $A_{(m)}$ defined by $\operatorname{cof}(L+k I)=A_{L+k I}=\sum_{m=1}^{n} A_{(m)} k^{m-1}$ are Killing tensors. Because the eigenfunctions of $L+k I$ are $\lambda^{i}+k$, the eigenfunctions of $A_{L+k I}$ are $\prod_{j \neq i}\left(\lambda^{j}+k\right)=\sum_{m=1}^{n} \sigma_{n-m}^{i} k^{m-1}$ with $\sigma_{m}^{i}$ the symmetric polynomial of order $m$ of $(n-1)$ variables $\lambda^{j}(j \neq i)$. So, the eigenfunctions of $A_{(m)}$ are the $\sigma_{n-m}^{i}$ which are functionally independent. From this follows also that the $A_{(m)}$ are functionally independent. Moreover, $A_{L+k I}$ has the same eigenforms as $L$, in other words the $d \lambda^{i}$ are simultaneously closed eigenforms for the $A_{(m)}$. The condition that the $H_{(m)}=\frac{1}{2} A_{(m)}^{i j} p_{i} p_{j}$ commute pairwise is also satisfied (see theorem 11). So, the tensors $A_{(m)}$ satisfy the Eisenhart conditions. In fact it can be shown that $A_{L+k I}=\sum_{m=1}^{n} K_{(m)} k^{n-m}=$ $\sum_{m=1}^{n} K_{(n-m+1)} k^{m-1}$ where $K_{(m)}$ is defined by $(2.5)$, i.e. $A_{(m)}=K_{(n-m+1)}$. 


\subsection{SCK tensors and projective equivalence}

Special conformal Killing tensors have another important application in the projective equivalence problem.

Suppose that we have a Riemannian manifold $(Q, g)$.

Definition 21. A (pseudo) Riemannian metric $\widetilde{g}$ is projectively (or geodesically) equivalent with $g$ if every geodesic of $g$, considered as an unparameterised curve, is a geodesic of $\widetilde{g}$.

The study of metrics with the same geodesics is very classical. It already started in the 19th century with Beltrami, Dini and Levi-Civita. The first author found the following interesting criterion for projective equivalence of two Riemann metrics:

$$
\widetilde{g}_{i j \mid k}=2 \frac{\partial \lambda}{\partial u^{k}} \widetilde{g}_{i j}+\frac{\partial \lambda}{\partial u^{i}} \widetilde{g}_{j k}+\frac{\partial \lambda}{\partial u^{j}} \widetilde{g}_{i k} \quad \text { for some } \lambda
$$

(for a more general description of the projective equivalence problem see chapter 4). In [5] it was proven that the projective equivalence of Riemannian metrics can be characterised by special conformal Killing tensors. Namely the projective equivalence of $g$ and $\widetilde{g}$ implies that the $(1,1)$ tensor $L$, implicitly defined by

$$
L_{i j}=\left(\frac{\operatorname{det} \widetilde{g}}{\operatorname{det} g}\right)^{1 / n+1} \widetilde{g}_{i j}^{-1}
$$

(lowering indices w.r.t $g$ ) is a special conformal Killing tensor. Conversely, if $L$ is an invertible special conformal Killing tensor, it is known that $g$ and $\widetilde{g}$ defined by

$$
\widetilde{g}_{i j}=(\operatorname{det} L)^{-1} L_{i j}^{-1}
$$

are projectively equivalent. Note that in this case

$$
\frac{\partial \lambda}{\partial u^{i}}=-\frac{1}{2} \frac{\partial \log \mu}{\partial q^{i}} \quad \text { with } \quad \mu=\operatorname{det} L=\left(\frac{\operatorname{det} g}{\operatorname{det} \widetilde{g}}\right)^{1 / n+1} .
$$

Starting from the geodesic equivalence of two Riemannian metrics, one can construct pairwise commuting integrals (see [39]). 
Theorem 12. Let $g$ and $\widetilde{g}$ be projectively equivalent. Denote by $G$ the linear operator with $G_{j}^{i}=g^{i k} \widetilde{g}_{k j}=(\operatorname{det} L)^{-1} L^{-1}$. Consider the characteristic polynomial

$$
\operatorname{det}(G-t I)=c_{0} t^{n}+c_{1} t^{n-1}+\ldots+c_{n} .
$$

Then the functions $I_{k}=g\left(S_{k} \mathbf{T}, \mathbf{T}\right) k=0, \ldots, n-1$ where

$$
S_{k}=\mu^{k+2} \sum_{i=0}^{k} c_{i} G^{k-i+1}
$$

are integrals for the geodesic flow of the metric $g$ and commute pairwise.

The first integral $I_{0}=\mu^{2} \widetilde{g}(\mathbf{T}, \mathbf{T})=(\operatorname{det} L) g\left(\mathbf{T}, L^{-1} \mathbf{T}\right)$ was already known to Painlevé (1897). An alternative way to derive first integrals is presented by Crampin in [11]. There it is shown that if we have two projectively equivalent Riemannian metrics, we can construct a quasi-Hamiltonian structure. As in section 2.5, we can consider then the special conformal Killing tensor $L+k I$ to find $n$ involutive first integrals $H_{(m)}$. In [11] it is also shown how the integrals $H_{(m)}$ and $I_{k}$ are related.

\subsection{SCK tensors and Poisson-Nijenhuis structures}

In this section we will explain the relation between the special conformal Killing tensors and Poisson-Nijenhuis structures, as presented in [8].

As an example of the Poisson-Nijenhuis structures, described in subsection 1.2.2, we consider the case that $M$ is a cotangent bundle, $M=T^{*} Q$, with its standard symplectic structure $\omega=d \theta=d p_{k} \wedge d q^{k}$. Suppose that $J=$ $J_{j}^{i}(q) \frac{\partial}{\partial q^{i}} \otimes d q^{j}$ is a type $(1,1)$ tensor field on $Q$ and consider the 2 -form

$$
\omega_{1}=\mathcal{L}_{J^{v}} \omega=d i_{J^{v}} d \theta
$$

where $J^{v}=J_{j}^{i} p_{i} \frac{\partial}{\partial p_{j}}$ is the vertical lift of $J$, which is a vector field on $T^{*} Q$. Now we can define the tensor $R$ by (1.7). Then $R$ is the complete lift $\widetilde{J}$ of $J$

$$
\widetilde{J}=J_{j}^{i}\left(\frac{\partial}{\partial q^{i}} \otimes d q^{j}+\frac{\partial}{\partial p_{j}} \otimes d p_{i}\right)+p_{k}\left(\frac{\partial J_{i}^{k}}{\partial q^{j}}-\frac{\partial J_{j}^{k}}{\partial q^{i}}\right) \frac{\partial}{\partial p_{i}} \otimes d q^{i}
$$

and $R$ is symmetric w.r.t. $\omega$. Moreover $d \omega_{1}=0$. Thus if we assume that $N_{J}=0$ (from which follows that $N_{\widetilde{J}}=0$ ), we have a Poisson-Nijenhuis structure. 
Note that for the generation of functions in involution, according to theorem 2 , one needs a solution $f$ of the equation $d d_{R} f=0$. Observe further that this is equivalent (from (1.9)) to requiring that $\mathcal{L}_{X_{f}} R=0$, i.e. $R$ is a recursion operator for the Hamiltonian system $X_{f}$.

Consider the particular case that $f=h=\frac{1}{2} g^{i j} p_{i} p_{j}$. In [8], Crampin, Sarlet and Thompson proved the following proposition.

Proposition 5. Let $g$ be a given metric tensor field on $Q$ and $h=\frac{1}{2} g^{i j} p_{i} p_{j}$ the corresponding kinetic energy Hamiltonian on $T^{*} Q$. Then, for a type $(1,1)$ tensor field $J$ on $Q$ to have the property $d d_{\widetilde{J}} h=0$, it is necessary and sufficient that $J$ is symmetric and parallel.

As a consequence of these conditions, the Nijenhuis torsion of $\widetilde{J}$ (and thus of $J$ ) will automatically vanish.

The next equation we look at is inspired by Magri's condition for separability (see equation (1.19)), namely we examine equation

$$
d d_{\widetilde{J}} h=d h \wedge d f
$$

with $h=\frac{1}{2} g^{i j} p_{i} p_{j}+V$ and $f$ a basic function. This equation can also be related to a gauged bi-differential calculus. Namely, suppose that $\left(d, d_{R}\right)$ is a bi-differential calculus and consider the following operators: $D_{1}=d$ and

$$
D_{2}\left[\begin{array}{l}
\alpha \\
\beta
\end{array}\right]=d_{R}\left[\begin{array}{l}
\alpha \\
\beta
\end{array}\right]+\left[\begin{array}{cc}
d f & 0 \\
d h & 0
\end{array}\right] \wedge\left[\begin{array}{l}
\alpha \\
\beta
\end{array}\right]=\left[\begin{array}{l}
d_{R} \alpha+d f \wedge \alpha \\
d_{R} \beta+d_{h} \wedge \alpha
\end{array}\right]
$$

for some fixed functions $f$ and $h$. $\left(D_{1}, D_{2}\right)$ forms a gauged bi-differential calculus with $m=2$ if $D_{1}^{2}=0,\left[D_{1}, D_{2}\right]=0$ and $D_{2}^{2}=0$. The first two conditions are automatically satisfied, but $D_{2}^{2}=0$ if and only if $f$ and $h$ satisfy (2.10) and $d d_{R} f=0$. The examination of equation (2.10) leads to the following interesting proposition.

Proposition 6. Let $g$ be a given metric on $Q$ and $V$ and $f$ functions on $Q$. Let $h$ be the Hamiltonian function on $T^{*} Q$ given by $h=\frac{1}{2} g^{i j} p_{i} p_{j}+V(q)$. A type $(1,1)$ tensor field $J$ on $Q$ will have the property $d d_{\widetilde{J}} h=d h \wedge d f$ if and only if
1. $J$ is symmetric;
2. $J$ is a special conformal Killing tensor with $f=\operatorname{tr} J$.
3. $V$ and $f$ satisfy $d d_{J} V=d V \wedge d f$. 
Remark that from these conditions follows that $N_{J}=0$ (see proposition 1) and $d d_{J} f=0$. This implies that all the conditions for the existence of a gauged bi-differential calculus of the type described above, are satisfied.

So much for the review of known results. As said in the introduction, we will now start with our attempt to generalise the constructions which led in the Riemannian case to the concept of 'special conformal Killing tensor'. As a first step (see chapter 3 ), we will develop a tangent bundle version of the theory discussed in section 2.7 about Poisson-Nijenhuis structures. This approach will lead to new insights and will show us how to proceed for the generalisation.

In chapter 4, we start from a given Lagrangian system and consider a type $(1,1)$ tensor $J$ along the tangent bundle projection (as apposed to a tensor on the base manifold) to generalise the various constructions of chapter 3 . In chapter 5 we look at the particular case of a Lagrangian coming from a Finsler metric where the tensor $J$ then is assumed to be homogeneous of degree zero in the velocities. We will focus on a certain condition which reduces to the special conformal Killing condition in case of a Riemann metric and a basic tensor. This condition will reappear in chapter 6 where we examine the projective equivalence of two Finsler metrics. We will also discuss the construction of a set of first integrals starting from this condition. 


\section{Chapter 3}

\section{A class of Poisson-Nijenhuis structures on a tangent bundle}

As we have seen in chapter 2, most of the applications of the special conformal Killing tensors are developed on a cotangent bundle. But some of them clearly come from meaningful questions about dynamical systems living on a tangent bundle. This is, for example, the case with the theory of cofactor pair systems (see section 2.5) where the physical background is a kinetic energy type Lagrangian for which admissible non-conservative forces are being sought such that the system has a family of first integrals in involution. As a first step in our attempt to generalise the theory of special conformal Killing tensors from Riemann to Finsler spaces, we will give in this chapter a tangent bundle version of section 2.7 where the relation between special conformal Killing tensors and Poisson-Nijenhuis structures is described (see our paper [44]). The calculus along the tangent bundle projection has already been fully developed and will be recalled in section 3.1. In the other sections of this chapter we examine in detail how the results of section 2.7 can be obtained in a natural way by pure tangent bundle techniques. There will arise some new, interesting properties and insights which will be helpful for the generalisations which follow in subsequent chapters.

\subsection{Calculus along the tangent bundle projection}

We will start this chapter with a summary of the papers [36] and [37] where the calculus along the tangent bundle projection $\tau: T Q \rightarrow Q$ is developed. Only the facts which will be needed in what follows, will be recalled.

Consider a system of second order autonomous ordinary differential equations $\ddot{q}^{i}=f^{i}(q, \dot{q})$ modelled by a vector field

$$
\Gamma=u^{i} \frac{\partial}{\partial q^{i}}+f^{i}(q, u) \frac{\partial}{\partial u^{i}}
$$


on $T Q$. $\Gamma$ defines a horizontal distribution on $T Q$ locally spanned by the vector fields

$$
H_{i}=\frac{\partial}{\partial q^{i}}-\Gamma_{i}^{j} \frac{\partial}{\partial u^{j}}, \quad \text { where } \quad \Gamma_{i}^{j}=-\frac{1}{2} \frac{\partial f^{j}}{\partial u^{i}} .
$$

$\mathcal{X}(\tau)$ denotes the $C^{\infty}(T Q)$-module of vector fields along $\tau$, i.e. sections of the pullback bundle $\tau^{*} T Q \rightarrow T Q$. Locally an element $X \in \mathcal{X}(\tau)$ is of the form

$$
X=X^{i}(q, u) \frac{\partial}{\partial q^{i}} .
$$

Each such an $X$ determines two vector fields on $T Q$, its horizontal lift $X^{H}$ where

$$
X^{H}=X^{i}\left(\frac{\partial}{\partial q^{i}}-\Gamma_{i}^{j} \frac{\partial}{\partial u^{j}}\right)=X^{i} H_{i},
$$

and its vertical lift $X^{V}$ given by

$$
X^{V}=X^{i} \frac{\partial}{\partial u^{i}}=X^{i} V_{i}
$$

We can also define horizontal and vertical lifts of a type $(1,1)$ tensor field $W$ along $\tau$ by

$$
\begin{aligned}
W^{H}\left(X^{V}\right)=W(X)^{V}, & W^{H}\left(X^{H}\right)=W(X)^{H}, \\
W^{V}\left(X^{V}\right)=0, & W^{V}\left(X^{H}\right)=W(X)^{V} .
\end{aligned}
$$

There exists a canonical vector field along $\tau$, the total time derivative $\mathbf{T}=$ $u^{i} \frac{\partial}{\partial q^{i}}$. Remark that $\mathbf{T}^{V}=\Delta$ with $\Delta$ the Liouville vector field, whereas $\mathbf{T}^{H}$ is a SODE which is usually called the SODE associated to the connection and does not need to be the one we started from. $S=I^{V}=\frac{\partial}{\partial u^{i}} \otimes d q^{i}$ is the canonical almost tangent structure or vertical endomorphism on $T Q$.

Interesting derivations and tensorial objects along $\tau$ are discovered by looking at the decomposition of Lie brackets of vector fields on $T Q$. We have, for example, that

$$
\begin{aligned}
& {\left[X^{V}, Y^{V}\right]=\left([X, Y]_{V}\right)^{V}} \\
& {\left[X^{H}, Y^{V}\right]=\left(\mathrm{D}_{X}^{H} Y\right)^{V}-\left(\mathrm{D}_{Y}^{V} X\right)^{H}} \\
& {\left[X^{H}, Y^{H}\right]=\left([X, Y]_{H}\right)^{H}+\mathcal{R}(X, Y)^{V} .}
\end{aligned}
$$

where

$$
[X, Y]_{V}=\mathrm{D}_{X}^{V} Y-\mathrm{D}_{Y}^{V} X, \quad[X, Y]_{H}=\mathrm{D}_{X}^{H} Y-\mathrm{D}_{Y}^{H} X
$$


$\mathcal{R}$ is the curvature of the non-linear connection, a vector valued 2 -form along $\tau$ given by

$$
\mathcal{R}=\frac{1}{2} \mathcal{R}_{j k}^{i} d q^{j} \wedge d q^{k} \otimes \frac{\partial}{\partial q^{i}}, \quad \text { where } \quad \mathcal{R}_{j k}^{i}=H_{k}\left(\Gamma_{j}^{i}\right)-H_{j}\left(\Gamma_{k}^{i}\right) .
$$

The degree zero derivations $\mathrm{D}_{X}^{H}$ and $\mathrm{D}_{X}^{V}$, called respectively the horizontal and vertical covariant derivative act on functions $F \in C^{\infty}(T Q)$ as

$$
\mathrm{D}_{X}^{H} F=X^{H}(F), \quad \mathrm{D}_{X}^{V} F=X^{V}(F),
$$

and are further fully determined by the following action on basic vector fields

$$
\mathrm{D}_{X}^{H} \frac{\partial}{\partial q^{i}}=X^{j} \Gamma_{j i}^{k} \frac{\partial}{\partial q^{k}}, \quad \mathrm{D}_{X}^{V} \frac{\partial}{\partial q^{i}}=0,
$$

where $\Gamma_{j k}^{i}=\frac{\partial \Gamma_{j}^{i}}{\partial u^{k}}$. The corresponding action on 1-forms is defined by duality. Notice that $D_{X}^{V} \mathbf{T}=X$ for every vector field $X$. The commutator relations of these derivatives are:

$$
\begin{aligned}
& {\left[\mathrm{D}_{X}^{V}, \mathrm{D}_{Y}^{V}\right]=\mathrm{D}_{[X, Y]_{V}}^{V},} \\
& {\left[\mathrm{D}_{X}^{V}, \mathrm{D}_{Y}^{H}\right]=\mathrm{D}_{\mathrm{D}_{X}^{H} Y}^{H}-\mathrm{D}_{\mathrm{D}_{Y}^{H} X}^{V}+\mu_{B(X, Y)},} \\
& {\left[\mathrm{D}_{X}^{H}, \mathrm{D}_{Y}^{H}\right]=\mathrm{D}_{[X, Y]_{H}}^{H}+\mathrm{D}_{\mathcal{R}(X, Y)}^{V}+\mu_{\operatorname{Rie}(X, Y)},}
\end{aligned}
$$

for arbitrary $X, Y \in \mathcal{X}(\tau)$. Here $B$ and Rie are type $(1,3)$ tensor fields along $\tau$ or, as they appear here, covariant 2-tensors taking values in the module of type $(1,1)$ tensors. For a general type $(1,1)$ tensor field $W, \mu_{W}$ is a derivation of the tensor algebra along $\tau$ of degree zero, whose action on functions is zero, while $\mu_{W}(Z)=W Z$ on vector fields $Z$ and $\mu_{W}(\alpha)=-W \alpha$ on 1-forms $\alpha$. To specify now the curvature tensors under consideration, we have for the so-called mixed curvature tensor $B$ that $B(X, Y) Z$ is symmetric in all three arguments and has coordinate expression:

$$
B=\Gamma_{j m l}^{k} d q^{l} \otimes d q^{j} \otimes\left(d q^{m} \otimes \frac{\partial}{\partial q^{k}}\right)
$$

where $\Gamma_{j m l}^{k}=\frac{\partial \Gamma_{j m}^{k}}{\partial u^{l}}$. The tensor Rie on the other hand (which is the Riemann curvature tensor in Riemannian geometry) is defined in general by

$$
\operatorname{Rie}(X, Y) Z=-\mathrm{D}_{Z}^{V} \mathcal{R}(X, Y)
$$


and its coordinate expression is:

$$
\text { Rie }=\frac{1}{2}\left(H_{k}\left(\Gamma_{l j}^{i}\right)-H_{l}\left(\Gamma_{k j}^{i}\right)+\Gamma_{k r}^{i} \Gamma_{l j}^{r}-\Gamma_{l r}^{i} \Gamma_{k j}^{r}\right) d q^{k} \wedge d q^{l} \otimes\left(d q^{j} \otimes \frac{\partial}{\partial q^{i}}\right) .
$$

Since $\mathrm{D}_{X}^{V}$ and $\mathrm{D}_{X}^{H}$ depend linear on $X$, one can define two operators $\mathrm{D}^{V}$ and $\mathrm{D}^{H}$ by the rule

$$
\left.X\lrcorner \mathrm{D}^{V} W=\mathrm{D}_{X}^{V} W, \quad X\right\lrcorner \mathrm{D}^{H} W=\mathrm{D}_{X}^{H} W
$$

where $W$ is an arbitrary tensor field along $\tau$. Remark that $\mathrm{D}^{V}$ and $\mathrm{D}^{H}$ are not derivations.

It is further worthwhile observing that one can introduce a kind of classical tensor calculus notation for the horizontal covariant derivative: taking as example a 2-covariant tensor $K$ along $\tau$, with components $K_{i j}$, we can put

$$
K_{i j \mid l}:=\left(\mathrm{D}_{\partial / \partial q^{l}}^{H} K\right)_{i j}=H_{l}\left(K_{i j}\right)-K_{i s} \Gamma_{l j}^{s}-K_{s j} \Gamma_{l i}^{s} .
$$

and for a type $(1,1)$ tensor $W$, we have

$$
W_{j \mid l}^{i}=\left(\mathrm{D}_{\partial / \partial q^{l}}^{H} W\right)_{j}^{i}=H_{l}\left(W_{j}^{i}\right)+W_{j}^{s} \Gamma_{s l}^{i}-W_{s}^{i} \Gamma_{j l}^{s}
$$

We shall occasionally use such a notation. Other brackets of interest are

$$
\left[\Gamma, X^{V}\right]=-X^{H}+(\nabla X)^{V}, \quad\left[\Gamma, X^{H}\right]=(\nabla X)^{H}+\Phi(X)^{V} .
$$

Here, $\nabla$ is the dynamical covariant derivative, which on functions acts like $\Gamma$ and further satisfies

$$
\nabla \partial / \partial q^{i}=\Gamma_{i}^{j} \partial / \partial q^{j}
$$

and

$$
\nabla d q^{i}=-\Gamma_{j}^{i} d q^{j}
$$

$\Phi$ is a type $(1,1)$ tensor along $\tau$, called the Jacobi endomorphism, with components

$$
\Phi_{j}^{i}=-\frac{\partial f^{i}}{\partial q^{j}}-\Gamma_{k}^{i} \Gamma_{j}^{k}-\Gamma\left(\Gamma_{j}^{i}\right)
$$

One can also introduce vertical and horizontal exterior derivations on scalar and vector-valued forms. Essentially, they are determined by the following 
action on functions $F \in C^{\infty}(T Q)$, vector fields $Z \in \mathcal{X}(\tau)$ and (scalar or vector-valued) 1-forms $W$ :

$d^{V} F(X):=\mathrm{D}_{X}^{V} F, \quad d^{V} Z(X):=\mathrm{D}_{X}^{V} Z, \quad d^{V} W(X, Y):=\mathrm{D}_{X}^{V} W(Y)-\mathrm{D}_{Y}^{V} W(X)$,

with similar defining relations for $d^{H}$. We need these exterior derivatives for example to show the following relations between the Jacobi endomorphism $\Phi$ and the curvature $\mathcal{R}$ :

$$
d^{V} \Phi=3 \mathcal{R}, \quad d^{H} \Phi=\nabla R .
$$

The dynamical covariant derivative $\nabla$ can be written as the following sum:

$$
\nabla=\mathrm{D}_{\mathbf{T}}^{H}+\mathrm{D}_{\nabla \mathbf{T}}^{V}-\mu_{d^{H} \mathbf{T}}
$$

Finally we recall that a SODE $\Gamma$ is Lagrangian if there exists a function $\ell$ on $T Q$ such that $\Gamma$ represents the Euler-Lagrange equations of $\ell$. The necessary and sufficient conditions (the Helmholtz conditions) can be formulated as follows: a SODE $\Gamma$ is locally Lagrangian if and only if there exists a nondegenerate symmetric type $(0,2)$ tensor field $g$ along $\tau$ satisfying

$$
\nabla g=0, \quad \mathrm{D}_{X}^{V} g(Y, Z)=\mathrm{D}_{Z}^{V} g(Y, X), \quad g(\Phi X, Y)=g(X, \Phi Y) .
$$

The Lagrangian $\ell$ and the tensor $g$ are related by $g=\mathrm{D}^{V} \mathrm{D}^{V} \ell$. In view of the commutator property

$$
\left[\nabla, \mathrm{D}_{X}^{V}\right]=\mathrm{D}_{\nabla X}^{V}-\mathrm{D}_{X}^{H}
$$

the Helmholtz conditions further imply that also

$$
\mathrm{D}_{X}^{H} g(Y, Z)=\mathrm{D}_{Z}^{H} g(Y, X) .
$$

The Poincaré-Cartan 2-form $\omega_{\ell}=d S(d \ell)=d d_{S} \ell$ of a Lagrangian $\ell$ on $T Q$ is the so-called Kähler lift $g^{K}$ of $g ; \omega_{\ell}$ vanishes on two vertical or two horizontal vector fields, while

$$
\omega_{\ell}\left(X^{V}, Y^{H}\right)=g(X, Y) .
$$

The Poincaré-Cartan 1-form $\theta_{\ell}=d_{S} \ell$ by the way, being a semi-basic form, can be viewed as a 1-form along $\tau$ as well and can then be written as $\theta_{\ell}=$ $d^{V} \ell$, so that $\theta_{\ell}\left(X^{V}\right)=0$ and $\theta_{\ell}\left(X^{H}\right)=\mathrm{D}_{X}^{V} \ell$. We also have that $\mathrm{D}_{X}^{V} \theta_{\ell}=$ $X\lrcorner g$. Observe, finally, that a necessary and sufficient condition for $\ell$ to be a Lagrangian for a SODE $\Gamma$ is that

$$
\nabla \theta_{\ell}=d^{H} \ell
$$

or equivalently

$$
d^{H} \ell=\frac{1}{2} d^{V}(\Gamma(\ell))
$$




\subsubsection{The Riemannian case}

Let $g$ be a symmetric, non-singular type $(0,2)$ tensor field on $Q$ and put $\ell=\frac{1}{2} g_{i j} u^{i} u^{j}$. The non-linear connection defined by the Euler-Lagrange equations then is the (linear) Levi-Civita connection of $g$, i.e. the connection coefficients $\Gamma_{j}^{i}$ are of the form

$$
\Gamma_{j}^{i}=\Gamma_{j k}^{i}(q) u^{k},
$$

where $\Gamma_{j k}^{i}$ are the classical Christoffel symbols. In this case, the deviation (= the difference between the given SODE and the one associated to the connection) $\nabla \mathbf{T}$ is zero. We have also that $\mathrm{D}_{X}^{H} \mathbf{T}=0$. These properties are easy to verify in coordinates, but we can also use the commutator property (3.18) to show in a coordinate free way that $\nabla \mathbf{T}=0$ and $\mathrm{D}_{X}^{V} \mathbf{T}=X$ imply that $\mathrm{D}_{X}^{H} \mathbf{T}=0$. Further we can say that the dynamical covariant derivative $\nabla=\mathrm{D}_{\mathbf{T}}^{H}$, so the relation between $\nabla$ and the classical covariant derivative $\left.\right|_{k}$ is in this case as follows:

$$
(\nabla J)_{j}^{i}=J_{j \mid k}^{i} u^{k} .
$$

Since $g$ is basic we have of course that $\mathrm{D}_{X}^{V} g=0$. Using commutator property (3.18) again, it follows also that $\mathrm{D}_{X}^{H} g=0, \forall X$. Note further that the Poincaré-Cartan 1-form $\theta_{\ell}$ can be expressed in terms of the metric as

$$
\left.\theta_{\ell}(X)=g(\mathbf{T}, X) \quad \text { or } \quad \theta_{\ell}=\mathbf{T}\right\lrcorner g .
$$

So, $\nabla \theta_{\ell}=0$. We should also keep in mind that $\ell=E_{\ell}$ is a first integral, so that $\nabla \ell=\Gamma(\ell)=0$. From (3.21) then follows that

$$
d^{H} \ell=0 .
$$

\subsection{The construction of a type $(1,1)$ tensor field $R$}

As said before, we want to get a tangent bundle approach to the theory about Poisson-Nijenhuis structures described in section 2.7. The difference between the tangent bundle and cotangent bundle is of course that the tangent bundle does not carry a canonical Poisson structure. However a symplectic form is available (and can be constructed by pure tangent bundle techniques) as soon as a regular Lagrangian is given, which could be for example the kinetic energy Lagrangian coming from a metric on $Q$. So, the tangent bundle approach makes perfectly sense. Moreover, we shall see 
that this tangent bundle version offers some new insights. We will derive for example some results valid for arbitrary Lagrangians, not just kinetic energy type ones. In this respect, we are to some extent joining the interest in Poisson structures on a tangent bundle which is also present in the work by Vaisman ([40], [50], [51]).

Suppose we are given a regular Lagrangian $\ell$ and type $(1,1)$ tensor field $J$ on a general manifold $Q$. We want to construct a type $(1,1)$ tensor field $R$, making use of formula (1.7). We therefore start with exploring the possible natural ways for constructing a second 2 -form $\omega_{1}$ from the given data on $T Q$.

We first look at the complete lift $J^{c}$ of $J$ on $T Q: \forall X, Y \in \mathcal{X}(Q)$

$$
J^{c}\left(X^{c}\right)=(J X)^{c}, \quad J^{c}\left(X^{V}\right)=(J X)^{V} .
$$

For the Nijenhuis torsion of $J^{c}$ we have,

$$
\begin{aligned}
& N_{J^{c}}\left(X^{V}, Y^{V}\right)=0, \\
& N_{J^{c}}\left(X^{c}, Y^{c}\right)=\left(N_{J}(X, Y)\right)^{c}, \\
& N_{J^{c}}\left(X^{V}, Y^{c}\right)=N_{J^{c}}\left(X^{c}, Y^{V}\right)=\left(N_{J}(X, Y)\right)^{V},
\end{aligned}
$$

from which it follows that $N_{J^{c}}=0 \Longleftrightarrow N_{J}=0$. In relation with the vertical endomorphism $S$, we find also the following properties of $J^{c}: J^{c}$ commutes with $S$, in the sense of endomorphisms on $\mathcal{X}(T Q)$, but also in the sense of the Nijenhuis bracket:

$$
\left[J^{c}, S\right]=0 .
$$

It follows that also the corresponding degree 1 derivations commute, meaning that:

$$
d_{S} d_{J^{c}}=-d_{J^{c}} d_{S} .
$$

Finally, it is easy to verify that for any $J$,

$$
N_{J^{c} S}=0 .
$$

In fact, we can make the following more complete statement in that respect, which is trivial to prove, and essentially says that $J^{c} S$ very much has the same properties as $S$.

Lemma 2. We have $\left(J^{c} S\right)^{2}=0$ and $N_{J^{c} S}=0$. Furthermore, if $J$ is non-singular, $J^{c} S$ determines an integrable almost tangent structure. 
Looking at the role which $S$ plays in the definition of the Poincaré-Cartan 2form $\omega_{\ell}=d d_{S} \ell$, it looks perfectly natural, given $J$ and the sort of alternative integrable almost tangent structure which it creates, to consider the closed 2 -from $\omega_{1}$, defined (with various ways of writing the same expression) by,

$$
\omega_{1}=d\left(S J^{c}(d \ell)\right)=d\left(S\left(d_{J^{c}} \ell\right)\right)=d\left(J^{c}\left(d_{S} \ell\right)\right)=d d_{J^{c} S} \ell
$$

And so, with $\ell$ and $J$ as data, the type $(1,1)$ tensor field $R$ which will carry our attention is defined by

$$
i_{R(\xi)} d d_{S} \ell=i_{\xi} d d_{J^{c} S} \ell \quad \forall \xi \in \mathcal{X}(T Q) .
$$

We know that it will define a Poisson-Nijenhuis structure if and only if $N_{R}=0$. Remark that the tensor field $R$ is directly related to the complete lift $\widetilde{J}$ (see 2.9 ) on $T^{*} Q$.

Proposition 7. We have that $R$ defined by (3.31) satisfies $R=L_{e}^{*} \tilde{J}$, where Leg :TQ $\rightarrow T^{*} Q$ denotes the Legendre transformation coming from the regular Lagrangian $\ell$.

Proof. The defining relation of $\tilde{J}$

$$
i_{\tilde{J} \xi} d \theta=i_{\xi} \mathcal{L}_{J^{v}} d \theta
$$

has the same structure as (3.31). It is easy to see from the coordinate expression that $i_{J^{v}} d \theta=\tilde{J} \theta$, so that (3.32) implies $i_{\tilde{J} \xi} d \theta=i_{\xi} d \tilde{J} \theta$. But it is equally trivial to verify in coordinates that $\operatorname{Leg}^{*} \tilde{J} \theta=J^{c} \theta_{\ell}=d_{J^{c} S} \ell$. The result then immediately follows from taking the pullback under Leg of the new representation of (3.32).

As an immediate consequence of the fact that $N_{\tilde{J}}=0 \Leftrightarrow N_{J}=0$, we now come to the following conclusion.

Proposition 8. $\quad N_{R}=0 \Longleftrightarrow N_{J}=0$.

The next objective now is to obtain a reasonably practical description of $R$, for example by recognising its action on complete and vertical lifts. In fact, we believe that it is better for general purposes, to make use of horizontal and vertical lifts, rather than complete and vertical lifts. For that, of course, one needs a connection, but there is one available, namely the non-linear connection associated to the Euler-Lagrange equations of $\ell$ (being secondorder differential equations on $T Q$ ). 
To begin with, we observe that

$$
\begin{aligned}
J^{c}\left(X^{V}\right) & =(J X)^{V} \\
J^{c}\left(X^{H}\right) & =(J X)^{H}+\nabla J(X)^{V} .
\end{aligned}
$$

So, $J^{c}=J^{H}+(\nabla J)^{V}$. It is easy to see that $\omega_{1}=d\left(J^{c} \theta_{\ell}\right)$ gives zero when evaluated on two vertical vector fields. Next we have, passing as before from $\theta_{\ell}$, regarded as 1-form on $T Q$, to its interpretation as 1-form along $\tau$,

$$
\begin{aligned}
\omega_{1}\left(X^{V}, Y^{H}\right) & =\mathcal{L}_{X^{V}}\left(\theta_{\ell}\left((J Y)^{H}\right)\right)-\theta_{\ell}\left(J^{c}\left(\left[X^{V}, Y^{H}\right]\right)\right) \\
& =\mathrm{D}_{X}^{V}\left(\theta_{\ell}(J Y)\right)-\theta_{\ell}\left(J\left(\mathrm{D}_{X}^{V} Y\right)\right)=\mathrm{D}_{X}^{V} \theta_{\ell}(J Y) .
\end{aligned}
$$

Because $g=\mathrm{D}^{V} \mathrm{D}^{V} \ell$ and $\theta_{\ell}=d^{V} \ell$, it follows that

$$
\omega_{1}\left(X^{V}, Y^{H}\right)=g(X, J Y) .
$$

Proceeding in the same way, we get

$$
\begin{aligned}
\omega_{1}\left(X^{H}, Y^{H}\right) \\
=\mathcal{L}_{X^{H}}\left(\theta_{\ell}\left((J Y)^{H}\right)\right)-\mathcal{L}_{Y^{H}}\left(\theta_{\ell}\left((J X)^{H}\right)\right)-\theta_{\ell}\left(J^{c}\left(\left[X^{H}, Y^{H}\right]\right)\right) \\
=\mathrm{D}_{X}^{H}\left(\theta_{\ell}(J Y)\right)-\mathrm{D}_{Y}^{H}\left(\theta_{\ell}(J X)\right)-\theta_{\ell}\left(J\left([X, Y]_{H}\right)\right) \\
=\mathrm{D}_{X}^{H}\left(J \theta_{\ell}(Y)\right)-\mathrm{D}_{Y}^{H}\left(J \theta_{\ell}(X)\right)-\theta_{\ell}\left(J\left(\mathrm{D}_{X}^{H} Y-\mathrm{D}_{Y}^{H} X\right)\right) \\
=\mathrm{D}_{X}^{H}\left(J \theta_{\ell}\right)(Y)-\mathrm{D}_{Y}^{H}\left(J \theta_{\ell}\right)(X) .
\end{aligned}
$$

It follows that

$$
\omega_{1}\left(X^{H}, Y^{H}\right)=d^{H}\left(J \theta_{\ell}\right)(X, Y) .
$$

Proposition 9. The type $(1,1)$ tensor field $R$, defined by (3.31) has the following structure:

$$
\begin{aligned}
& R\left(X^{V}\right)=(\bar{J} X)^{V} \\
& R\left(X^{H}\right)=(J X)^{H}+(U X)^{V}
\end{aligned}
$$

where $\bar{J}$ is the transpose of $J$ with respect to $g=\mathrm{D}^{V} \mathrm{D}^{V} \ell$, i.e. $g(J X, Y)=$ $g(X, \bar{J} Y)$, and $U$ is the tensor field along $\tau$, determined by

$$
g(U X, Y)=d^{H}\left(J \theta_{\ell}\right)(X, Y) .
$$


PROOF. It is sufficient to take horizontal and vertical lifts of basic vector fields for finding the tensorial structure of $R$. We have $\omega_{\ell}\left(R\left(X^{V}\right), Y^{V}\right)=0$ and

$$
\omega_{\ell}\left(R\left(X^{V}\right), Y^{H}\right)=\omega_{1}\left(X^{V}, Y^{H}\right)=g(J Y, X)=g(Y, \bar{J} X)=g^{K}\left((\bar{J} X)^{V}, Y^{H}\right),
$$

from which (4.7) follows. Likewise

$$
\omega_{\ell}\left(R\left(X^{H}\right), Y^{V}\right)=-g(J X, Y)=g^{K}\left((J X)^{H}, Y^{V}\right),
$$

from which it follows that $R\left(X^{H}\right)=(J X)^{H}+(U X)^{V}$, for some $U$. Subsequently, using (3.20) and (3.36),

$$
\omega_{\ell}\left(R\left(X^{H}\right), Y^{H}\right)=\omega_{\ell}\left((U X)^{V}, Y^{H}\right)=g(U X, Y)=d^{H}\left(J \theta_{\ell}\right)(X, Y),
$$

which completes the proof.

Note that it follows from the skew-symmetry of the right-hand side in (3.39), that $\bar{U}=-U$. Further properties of $R$ are listed in the next three propositions.

Proposition 10. We have $R=J^{c} \Longleftrightarrow J=\bar{J}$ and $U=\nabla J$.

Proof. The result follows immediately from comparison of (3.37-3.38) with (3.33-3.34).

A natural question which arises is whether $R$, in general, could commute with $S$, just as $J^{c}$ does, either in the algebraic sense or with respect to the Nijenhuis bracket.

Proposition 11. $R S=S R \Longleftrightarrow J=\bar{J}$.

Proof. Using $S\left(X^{V}\right)=0$ and $S\left(X^{H}\right)=X^{V}$, the result follows immediately from the characterisation of $R$ in Proposition 9.

Proposition 12. Assuming $J=\bar{J}$, so that $R S=S R$, we have $[R, S]=$ $0 \Longleftrightarrow d^{V} U=d^{H} J$.

Proof. That $[R, S]$ vanishes on two vertical lifts is trivial. Again, it suffices for such calculations to consider lifts of basic vector fields (vector fields on $Q$ ), rather than vector fields along $\tau$. Since $J$ is basic as well, one then 
easily verifies, making use of the bracket relations (3.3) and (3.4), that also $[R, S]\left(X^{V}, Y^{H}\right)=0$. Next, we have

$$
\begin{aligned}
{[R, S]\left(X^{H}, Y^{H}\right)=} & {\left[(J X)^{H}+(U X)^{V}, Y^{V}\right]+\left[X^{V},(J Y)^{H}+(U Y)^{V}\right] } \\
& +2 R S\left([X, Y]_{H}{ }^{H}\right)-R\left(\left[X^{V}, Y^{H}\right]+\left[X^{H}, Y^{V}\right]\right) \\
& -S\left(\left[(J X)^{H}+(U X)^{V}, Y^{H}\right]+\left[X^{H},(J Y)^{H}+(U Y)^{V}\right]\right) .
\end{aligned}
$$

Using the bracket relations (3.3-3.5), plus the fact that $J$, and by assumption also $X$ en $Y$, are basic, this readily reduces to

$$
\begin{aligned}
& {[R, S]\left(X^{H}, Y^{H}\right)=} \\
& \quad\left(\mathrm{D}_{X}^{V} U(Y)-\mathrm{D}_{Y}^{V} U(X)\right)^{V}+\left(J\left(\mathrm{D}_{X}^{H} Y-\mathrm{D}_{Y}^{H} X\right)\right)^{V}+\left(\mathrm{D}_{Y}^{H}(J X)-\mathrm{D}_{X}^{H}(J Y)\right)^{V},
\end{aligned}
$$

which in view of properties such as $(3.14)$ for type $(1,1)$ tensors, can be written as

$$
[R, S]\left(X^{H}, Y^{H}\right)=\left(d^{V} U(X, Y)-d^{H} J(X, Y)\right)^{V} .
$$

The result now follows.

\subsubsection{The Darboux-Nijenhuis coordinates of $R$}

We have seen in subsection 1.2.2 that on a general (regular) Poisson-Nijenhuis manifold of dimension $2 n$, if the recursion operator $R$ has $n$ distinct eigenvalues, there exist so-called Darboux-Nijenhuis coordinates, which diagonalise $R$ and are at the same time Darboux coordinates for the symplectic form. This will apply in particular to the general situation on $T Q$, described here. We wish to investigate here in some detail what the structure is of the eigenspaces of our $R$ and how the construction of Darboux-Nijenhuis coordinates works when the eigenvalues are maximally distinct.

We begin by establishing results which are valid without special assumptions on the type $(1,1)$ tensor $J$ on $Q$, except that we will only consider real eigenvalues.

Lemma 3. If $\xi=X^{H}+Y^{V}$ is an eigenvector of $R$, corresponding to the eigenvalue $\lambda$, then

$$
J X=\lambda X \quad \text { and } \quad U X+\bar{J} Y=\lambda Y .
$$

It follows in particular that $X$ is an eigenvector of $J$. 
Proof. Using the characterisation of $R$ as described by (3.37-3.38), it is immediate to see that $R \xi=\lambda \xi$ is equivalent to the two relations (3.40).

Lemma 4. $J$ and $\bar{J}$ have the same eigenvalues. In fact, if $X$ is an eigenvector of $J$, then $X\lrcorner g$ is an eigenform of $\bar{J}$ with the same eigenvalue.

Proof. Suppose that $X$ is an eigenvector of $J$ corresponding to the eigenvalue $\lambda$, i.e. $J_{j}^{i} X^{j}=\lambda \delta_{j}^{i} X^{j}=\lambda X^{i}$. Multiplying this with $g_{i k}$, we find

$$
\bar{J}_{k}^{s} g_{s j} X^{j}=g_{i k} J_{j}^{i} X^{j}=\lambda g_{j k} X^{j}=\lambda \delta_{k}^{s} g_{s j} X^{j} .
$$

So, $X\lrcorner g$ is an eigenform of $\bar{J}$ corresponding to the eigenvalue $\lambda$.

Lemma 5. Suppose that $J$ is non-degenerate and has $n$ distinct eigenvalues (which then are non-zero). Then, if $J X=\lambda X$, there exists a vector field $Y$ along $\tau$, such that $\bar{J} Y=\lambda Y-U X$.

Proof. From $g(J X, Y)=g(X, \bar{J} Y)=\lambda g(X, Y)$, it follows that $g(X, \bar{J} Y-$ $\lambda Y)=0, \forall Y$. Extending $X=X_{1}$ to an orthogonal frame $\left\{X_{1}, \ldots, X_{n}\right\}=$ $\left\{X_{1}, X_{\alpha}\right\}$ for $g$, and putting $Y=a^{i} X_{i}$, it follows that $a^{i}\left(\bar{J} X_{i}-\lambda X_{i}\right) \in$ sp $\left\{X_{\alpha}\right\}, \forall a^{i}$, which implies that

$$
\bar{J} X_{i}=\lambda X_{i}+b_{i}^{\alpha} X_{\alpha}, \quad i=1, \ldots, n
$$

for some functions $b_{i}^{\alpha}$. We know that $\lambda$ is an eigenvalue of $\bar{J}$ as well, and that its eigenvalues are distinct. Hence, there exists a unique vector field of the form $X_{1}+c^{\alpha} X_{\alpha}$ which spans the kernel of $\bar{J}-\lambda I$. But $(\bar{J}-\lambda I)\left(X_{1}+c^{\alpha} X_{\alpha}\right)=$ $\left(b_{1}^{\alpha}+c^{\beta} b_{\beta}^{\alpha}\right) X_{\alpha}$, so the fact that unique functions $c^{\beta}$ exist which make this zero implies that $\operatorname{det}\left(b_{\beta}^{\alpha}\right) \neq 0$. Now consider the equation $\bar{J} Y=\lambda Y-U X$ for the unknown $Y=a^{i} X_{i} \in \mathcal{X}(\tau)$. Since $g(U X, Y)$ is skew-symmetric in $X, Y$, we know that $g\left(U X_{1}, X_{1}\right)=0$ and thus $U X_{1}=d^{\alpha} X_{\alpha}$ for some functions $d^{\alpha}$. The equation for $Y$ can now be written in the form $a^{\beta} b_{\beta}^{\alpha}=-d^{\alpha}-a^{1} b_{1}^{\alpha}$ and clearly has a unique solution for the $a^{\beta}$ for each arbitrary choice of $a^{1}$.

Proposition 13. Let $J$ be diagonalisable with distinct non-zero eigenvalues. Then a complete set of eigenvectors of $R$ defined in proposition 9 can be constructed as follows: (i) let $X_{i}$ denote the eigenvector of $J$ with eigenvalue $\lambda_{i}$ and $Z_{i}$ the eigenvector of $\bar{J}$ with the same eigenvalue; (ii) for each $X_{i}$, construct a vector $Y_{i}$ such that $\bar{J} Y_{i}=\lambda_{i} Y_{i}-U X_{i}$. Then $Z_{i}{ }^{V}$ and $X_{i}{ }^{H}+Y_{i}{ }^{V}$ are eigenvectors of $R$, corresponding to the eigenvalue $\lambda_{i}$. 
Proof. We have

$$
\begin{aligned}
R\left(Z_{i}{ }^{V}\right) & =\left(\bar{J} Z_{i}\right)^{V}=\lambda_{i} Z_{i}{ }^{V}, \\
R\left(X_{i}{ }^{H}+Y_{i}{ }^{V}\right) & =\left(J X_{i}\right)^{H}+\left(U X_{i}\right)^{V}+\left(\bar{J} Y_{i}\right)^{V}=\lambda_{i}\left(X_{i}{ }^{H}+Y_{i}{ }^{V}\right),
\end{aligned}
$$

from which the result follows.

Here ends the investigation of the purely algebraic aspects. It was proven in the fundamental paper of Frölicher and Nijenhuis [21] that if $J$ is (algebraically) diagonalisable and the eigenvalues have constant multiplicity, then the necessary and sufficient condition for diagonalisability in coordinates is that $\mathcal{H}_{J}=0$, where the Haantjes tensor $\mathcal{H}_{J}$ can be defined by

$$
\mathcal{H}_{J}(X, Y)=J^{2} N_{J}(X, Y)+N_{J}(J X, J Y)-J N_{J}(J X, Y)-J N_{J}(X, J Y) .
$$

Now let us assume that $J$ is diagonalisable with $n$ distinct non-zero eigenvalues and $N_{J}=0$. Obviously, the vanishing of the Nijenhuis torsion implies $\mathcal{H}_{J}=0$, so $J$ is diagonalisable in coordinates. Moreover, evaluating $N_{J}$ on eigenvectors $X$ and $Y$ belonging to different eigenvalues $\lambda, \mu$, gives

$$
0=N_{J}(X, Y)=(\lambda-\mu)(X(\mu) Y+Y(\lambda) X)
$$

so that $X(\mu)=Y(\lambda)=0$. Hence, in coordinates which diagonalise $J$, the eigenvalues will only depend on the coordinates of the corresponding eigendistribution, which is the meaning of saying that $J$ is separable in coordinates. Conversely, if $J$ is separable, one can verify in such coordinates that $N_{J}=0$.

Proposition 14. Let $J$ be a type $(1,1)$ tensor on $Q$, diagonalisable with $n$ distinct non-zero eigenvalues, then $N_{J}=0$ if and only if $J$ is separable in coordinates.

Note in passing that the tools for studying such issues when $J$ would more generally be a tensor field along $\tau$ have been developed in [36].

Now to understand the procedure which leads to Darboux-Nijenhuis coordinates for $R$ on $T Q$, we need to look at the expression of $R$ in coordinates basis. It reads

$$
R=J_{j}^{i} \frac{\partial}{\partial q^{i}} \otimes d q^{j}+\bar{J}_{j}^{i} \frac{\partial}{\partial u^{i}} \otimes d u^{j}+\left(U_{j}^{i}+\bar{J}_{k}^{i} \Gamma_{j}^{k}-J_{j}^{k} \Gamma_{k}^{i}\right) \frac{\partial}{\partial u^{i}} \otimes d q^{j} .
$$

The following procedure now will lead to Darboux-Nijenhuis coordinates. We first perform the Legendre transform $(q, u) \rightarrow(q, p=\partial \ell / \partial u)$. Even 
though this is to be regarded here as a change of coordinates on $T Q$, the result will be that $R$ acquires the form of the complete lift $\tilde{J}$ on $T^{*} Q$ as given by (2.9). The 2 -form $\omega_{\ell}$ meanwhile will already take its canonical form in the $(x, p)$ coordinates. Now, assuming that $J$ has distinct eigenvalues and zero Nijenhuis torsion, we know that there exists a coordinate change on $Q$ which will diagonalise the expression $J_{j}^{i}\left(\partial / \partial q^{i} \otimes d q^{j}\right)$ in such a way that the eigenvalues depend on at most one new coordinate $q^{\prime}$. The resulting point transformation $(q, u) \rightarrow\left(q^{\prime}, u^{\prime}\right)$ on $T Q$, when expressed in the non-tangent bundle variables $(q, p)$, formally is a 'canonical transformation' $(q, p) \rightarrow$ $\left(q^{\prime}, p^{\prime}\right)$, i.e. it defines another Darboux chart for the symplectic form $\omega_{\ell}$ and it will have the additional effect of diagonalising $R$ (and $R$ will be separable in coordinates).

From a tangent bundle point of view, the first step in this procedure is rather unnatural, because it is not a tangent bundle change of coordinates. At first sight, it may look like one should nevertheless not change the order of the operations, because even though $J$ and $\bar{J}$ have the same eigenvalues, a coordinate transformation which diagonalises $J$ will generally not at the same time diagonalise $\bar{J}$. However, the two coordinate changes under consideration here are of course of a quite special type: a Legendre transformation which does not change $q$ but changes the fibre coordinate, and a point transformation. It is clear that such coordinate changes commute, so one can just as well diagonalise $J$ first and then the subsequent Legendre transform will not destroy the diagonal form of $J$, will bring $\omega_{\ell}$ in canonical form, and at the same time will take care of the diagonalisation of $\bar{J}$.

That the reversed procedure is somewhat more natural for the tangent bundle set-up may become clear in the special case that $J$ is symmetric. It then follows from $g_{i j} J_{k}^{i}=g_{i k} J_{j}^{i}$ that in coordinates which diagonalise $J$, we will have $g_{i j} \delta_{k}^{i} \lambda^{(k)}=g_{i k} \delta_{j}^{i} \lambda^{(j)}$ or $g_{k j}\left(\lambda^{(k)}-\lambda^{(j)}\right)=0$ and thus follows that $g_{k j}=0$ for $j \neq k$. This gives useful information also when there is no urge to pass to Darboux-Nijenhuis coordinates: it means that in coordinates which diagonalise $J$, the given Lagrangian will be of the form $\ell=\sum_{i} g_{i i}(q) u^{i}=\sum_{i} \ell^{i}\left(q, u^{i}\right)$, where $\ell^{i}$ depends on $u^{i}$ only. So, the Lagrangian $\ell$ will separate with respect to the velocity variables.

\subsection{Investigation of $\mathcal{L}_{\Gamma} R$}

An obvious question to ask is: under which circumstances is $R$ truly a recursion operator for symmetries of $\Gamma$. For that, we compute $\mathcal{L}_{\Gamma} R$. Taking 
the Lie derivative with respect to $\Gamma$ of the defining relations (3.37-3.38) and making use also of the properties (3.13), we find

$$
\begin{aligned}
\mathcal{L}_{\Gamma} R\left(X^{V}\right) & =\left[\Gamma, R\left(X^{V}\right)\right]-R\left[\Gamma, X^{V}\right] \\
& =\left[\Gamma, \bar{J} X^{V}\right]+R\left(X^{H}-\nabla X^{V}\right) \\
& =(J-\bar{J})(X)^{H}+(U+\nabla \bar{J})(X)^{V}
\end{aligned}
$$

and in the same way

$$
\mathcal{L}_{\Gamma} R\left(X^{H}\right)=(\nabla J-U)(X)^{H}+(\nabla U+\Phi J-\bar{J} \Phi)(X)^{V} .
$$

The following, therefore, is an interesting immediate result.

Proposition 15. $\quad \mathcal{L}_{\Gamma} R=0 \Longleftrightarrow J=\bar{J}, \quad U=\nabla J=0, \quad \Phi J=J \Phi$.

So, in this case $R=J^{c}$. To find an alternative characterisation for the vanishing of $\mathcal{L}_{\Gamma} R$, we observe first that $\Gamma$, by construction, is the Hamiltonian vector field associated to the energy function $E_{\ell}=\Delta(\ell)-\ell$, with respect to the symplectic form $\omega_{\ell}=d \theta_{\ell}$, i.e. we have

$$
i_{\Gamma} d \theta_{\ell}=-d E_{\ell}
$$

So, equation (1.9) implies that the invariance of $R$ is equivalent to having $d d_{R} E_{\ell}=0$. As already explained, this forms the start of a sequence of functions if $\left(d, d_{R}\right)$ is a bi-differential calculus (see theorem 2 ).

Now we want to come to the special conformal killing tensor condition. For that purpose, we now look at the interesting case of non-vanishing $\mathcal{L}_{\Gamma} R$. By analogy with what was described in section 2.7 , we examine the assumption that for some basic function $f$,

$$
d d_{R} E_{\ell}=d f \wedge d E_{\ell}
$$

Via the equality (1.9), this assumption is equivalent to stating that (cf. proposition 5.3 in [8])

$$
\mathcal{L}_{\Gamma} R=\Gamma \otimes d f-\xi_{f} \otimes d E_{\ell},
$$

where $\xi_{f}$ is the Hamiltonian vector field associated to $f$. It easily follows, from $i_{S \xi_{f}} \omega_{\ell}=-S\left(i_{\xi_{f}} \omega_{\ell}\right)=S(d f)=0$, that $\xi_{f}$ is vertical, say $\xi_{f}=X_{f}^{V}$ for some $X_{f}$ along $\tau$. Then,

$$
i_{\xi_{f}} \omega_{\ell}\left(Y^{H}\right)=g^{K}\left(X_{f}^{V}, Y^{H}\right)=g\left(X_{f}, Y\right)=-Y^{H}(f) .
$$


Hence, in terms of fields along the projection $\tau, X_{f}$ is defined by

$$
\left.g\left(X_{f}, Y\right)=-Y^{H}(f)=-d^{H} f(Y) \quad \text { or } \quad X_{f}\right\lrcorner g=-d^{H} f .
$$

In the next subsection we specialise to the case that the Lagrangian comes from a (pseudo) Riemannian metric on $Q$, and will focus most of the attention on the characterisation of so-called special conformal Killing tensors in their tangent bundle manifestation.

\subsection{When the base manifold is Riemannian}

Let $g$ be a given metric tensor field on $Q$ and $\ell=\frac{1}{2} g_{i j} u^{i} u^{j}$, the corresponding kinetic energy Lagrangian on $T Q$. We now look at the characterisation of $R$ in this context, more particularly the specification of the tensor field $U$.

Using (3.24), we have

$$
\begin{aligned}
& d^{H}\left(J \theta_{\ell}\right)(X, Y) \\
& \quad=\mathrm{D}_{X}^{H}\left(J \theta_{\ell}\right)(Y)-\mathrm{D}_{Y}^{H}\left(J \theta_{\ell}\right)(X) \\
& \quad=\mathrm{D}_{X}^{H}(g(\mathbf{T}, J Y))-g\left(\mathbf{T}, J\left(\mathrm{D}_{X}^{H} Y\right)\right)-\mathrm{D}_{Y}^{H}(g(\mathbf{T}, J X))+g\left(\mathbf{T}, J\left(\mathrm{D}_{Y}^{H} X\right)\right) .
\end{aligned}
$$

Taking into account that $\mathrm{D}_{X}^{H} g=0$ and $\mathrm{D}_{X}^{H} \mathbf{T}=0$, we conclude that $U$ is determined by

$$
g(U X, Y)=g\left(\mathbf{T}, \mathrm{D}_{X}^{H} J(Y)-\mathrm{D}_{Y}^{H} J(X)\right)=g\left(\mathbf{T}, d^{H} J(X, Y)\right) .
$$

In coordinates, it is now convenient to work with the adapted frame of horizontal and vertical vector fields on $T Q$ and their dual 1-forms, which are

$$
\left\{H_{i}=\frac{\partial}{\partial q^{i}}-\Gamma_{i}^{k} \frac{\partial}{\partial u^{k}}, V_{i}=\frac{\partial}{\partial u^{i}}\right\}, \quad\left\{d q^{i}, \eta^{j}=d u^{j}+\Gamma_{k}^{j} d q^{k}\right\} .
$$

The coordinate expression for $R$ then becomes

$$
R=J_{j}^{i} H_{i} \otimes d q^{j}+\bar{J}_{j}^{i} V_{i} \otimes \eta^{j}+U_{j}^{i} V_{i} \otimes d q^{j},
$$

where

$$
U_{j}^{i}=g^{i k}\left(J_{k \mid j}^{m}-J_{j \mid k}^{m}\right) g_{m l} u^{l} .
$$

It is also interesting to have intrinsic expressions which implicitly determine the vertical and horizontal covariant derivatives of $U$. 
Proposition 16. For $Z \in \mathcal{X}(\tau), \mathrm{D}_{Z}^{V} U$ and $\mathrm{D}_{Z}^{H} U$ are determined by

$$
\begin{aligned}
& g\left(\mathrm{D}_{Z}^{V} U(X), Y\right)=g\left(Z, \mathrm{D}_{X}^{H} J(Y)-\mathrm{D}_{Y}^{H} J(X)\right), \\
& g\left(\mathrm{D}_{Z}^{H} U(X), Y\right)=g\left(\mathbf{T}, \mathrm{D}^{H} \mathrm{D}^{H} J(Z, X, Y)-\mathrm{D}^{H} \mathrm{D}^{H} J(Z, Y, X)\right),
\end{aligned}
$$

Proof. To prove expression (3.54), we compute the $\mathrm{D}_{Z}^{V}$ derivative of (3.50). Because $\mathrm{D}_{Z}^{V} g=0$ and $\mathrm{D}_{Z}^{V} \mathbf{T}=Z$ we get

$$
\begin{aligned}
& g\left(\mathrm{D}_{Z}^{V} U(X), Y\right) \\
&=\mathrm{D}_{Z}^{V}(g(U X, Y))-g\left(U\left(\mathrm{D}_{Z}^{V} X\right), Y\right)-g\left(U X, \mathrm{D}_{Z}^{V} Y\right) \\
&= \mathrm{D}_{Z}^{V}\left(g\left(\mathbf{T}, d^{H} J(X, Y)\right)\right)-g\left(\mathbf{T}, d^{H} J\left(\mathrm{D}_{Z}^{V} X, Y\right)\right)-g\left(\mathbf{T}, d^{H} J\left(X, \mathrm{D}_{Z}^{V} Y\right)\right) \\
&= g\left(Z, d^{H} J(X, Y)\right)+g\left(\mathbf{T}, \mathrm{D}_{Z}^{V} \mathrm{D}_{X}^{H} J(Y)-\mathrm{D}_{\mathrm{D}_{Z}^{V} X}^{H} J(Y)\right) \\
&-g\left(\mathbf{T}, \mathrm{D}_{Z}^{V} \mathrm{D}_{Y}^{H} J(X)-\mathrm{D}_{\mathrm{D}_{Z}^{V} Y}^{H} J(X)\right)
\end{aligned}
$$

where $\mathrm{D}_{Z}^{V} \mathrm{D}_{X}^{H} J-\mathrm{D}_{\mathrm{D}_{Z}^{V} X}^{H} J=\mathrm{D}_{X}^{H} \mathrm{D}_{Z}^{V} J-\mathrm{D}_{\mathrm{D}_{X}^{H} Z}^{V} J=0$ (see commutator property (3.9) with $B=0$ ). For expression (3.55) we have to take into account that $\mathrm{D}_{Z}^{H} g=0$ and $\mathrm{D}_{Z}^{H} \mathbf{T}=0$ and we find

$$
\begin{aligned}
g & \left(\mathrm{D}_{Z}^{H} U(X), Y\right) \\
& =\mathrm{D}_{Z}^{H}(g(U X, Y))-g\left(U \mathrm{D}_{Z}^{H} X, Y\right)-g\left(U X, \mathrm{D}_{Z}^{H} Y\right) \\
& =\mathrm{D}_{Z}^{H}\left(g\left(\mathbf{T}, d^{H} J(X, Y)\right)-g\left(\mathbf{T}, d^{H} J\left(\mathrm{D}_{Z}^{H} X, Y\right)\right)-g\left(\mathbf{T}, d^{H} J\left(X, \mathrm{D}_{Z}^{H} Y\right)\right)\right. \\
& =g\left(\mathbf{T}, \mathrm{D}^{H} \mathrm{D}^{H} J(Z, X, Y)-\mathrm{D}^{H} \mathrm{D}^{H} J(Z, Y, X)\right)
\end{aligned}
$$

where we have used the fact that (see definition (3.12))

$$
\mathrm{D}^{H} \mathrm{D}^{H} J(Z, X, Y)=\mathrm{D}_{Z}^{H} \mathrm{D}_{X}^{H} J(Y)-\mathrm{D}_{\mathrm{D}_{Z}^{H} X}^{H} J(Y) .
$$

Let us come back in this case of particular interest to the invariance of $R$, or the more general assumption (3.48). We know from proposition 15 that $\mathcal{L}_{\Gamma} R=0$ is equivalent with saying that $J$ is symmetric, parallel, commutes with the Jacobi endomorphism $\Phi$ and that $U$ vanishes. Or alternatively that $d d_{R} E_{\ell}=0$. But in proposition 5 it is shown that $d d_{R} E_{\ell}=0$ (which is the same as saying that $d d_{\widetilde{J}} h=0$ if $h$ is the corresponding Hamiltonian) is equivalent to $J=\bar{J}$ and $\nabla J=0$. So, we have the following maybe somewhat surprising result, for which it is worth giving a direct proof.

Proposition 17. Suppose that $J$ is a type $(1,1)$ tensor on a Riemannian manifold $Q$. If $J$ is symmetric and parallel, it will automatically commute with the Jacobi-endomorphism $\Phi$ and $U$ defined by (3.50) will vanish. 
Proof. If $\nabla J=0$, it follows from (3.23) that $\left.J_{j}^{i}\right|_{k}=0$ and so that $\mathrm{D}_{X}^{H} J=0$ for all $X$. Thus equation (3.50) implies that also $U=0$. The property about the Jacobi-endomorphism can be proven making use of an integrability analysis (see appendix A in [44]). A much simpler proof however goes as follows: we have that $J$ is symmetric and further we see that $J$ is the Hessian of a function:

$$
\mathcal{J}=\frac{1}{2} J_{i j} u^{i} u^{j} \quad \text { or in intrinsic terms } \quad \mathcal{J}=g(J \mathbf{T}, \mathbf{T})=\left(J \theta_{\ell}\right)(\mathbf{T})
$$

It follows from $\nabla \mathbf{T}=0, \nabla \theta_{\ell}=0$ and $\nabla J=0$ that $\mathcal{J}$ is a first integral. Moreover we have that

$$
d^{H} \mathcal{J}=d^{V} \nabla \mathcal{J}-\nabla d^{V} \mathcal{J}=-\nabla\left(J \theta_{\ell}\right)=0 .
$$

Using relation (3.21), we can conclude that $\mathcal{J}$ is a Lagrangian for the system. Therefore its Hessian $J$ will commute with $\Phi$.

Now assume that (3.48) holds for some $f \in C^{\infty}(Q)$ and where $\xi_{f}=X_{f}{ }^{V}$, with $X_{f}$ defined by (3.49). Remember further that, in the present situation, we have $E_{\ell}=\ell$ and $\Gamma=\mathbf{T}^{H}$.

Theorem 13. Under the present circumstances, the tensor field $\mathcal{L}_{\Gamma} R$ is of the form (3.48) if and only if $J=\bar{J}$ and further satisfies

$$
\nabla J=\frac{1}{2}\left(\mathbf{T} \otimes d^{H} f-X_{f} \otimes \theta_{\ell}\right) .
$$

In addition, $U$ then is of the form

$$
U=-\frac{1}{2}\left(\mathbf{T} \otimes d^{H} f+X_{f} \otimes \theta_{\ell}\right),
$$

and $J$ further has the property

$$
\Phi J-J \Phi=\frac{1}{2}\left(\mathbf{T} \otimes \nabla d^{H} f+\nabla X_{f} \otimes \theta_{\ell}\right) .
$$

Finally, the tensor field $R$ itself then is given by

$$
R=J^{c}-\Delta \otimes d f .
$$

Proof. The right-hand side of (3.48), when evaluated on some $X^{V}$, results in $-X^{V}\left(E_{\ell}\right) X_{f}^{V}=-\theta_{\ell}(X) X_{f}^{V}$. Comparison with (3.44) shows that this requires $J$ to be symmetric, plus the condition that

$$
U=-\nabla J-X_{f} \otimes \theta_{\ell} .
$$


Proceeding in the same way for an arbitrary horizontal argument $X^{H}$, comparison with (3.45) reveals the requirements

$$
U=\nabla J-\mathbf{T} \otimes d^{H} f
$$

and

$$
\nabla U+\Phi J-J \Phi=-X_{f} \otimes d^{H} \ell=0 .
$$

Compatibility of the two expressions for $U$ above, immediately leads to the conclusions (3.56) and (3.57). The first of these puts a restriction on $J$, while the second in fact is then automatically satisfied. To see this, we compute $d^{V} \nabla J$ from (3.56). Now $d^{V} d^{H} f=-d^{H} d^{V} f=0, d^{V} \mathbf{T}=I$ (the identity tensor), $d^{v} \theta_{\ell}=d^{V} d^{V} \ell=0$ and finally also $d^{V} X_{f}=0$ from (3.49). It follows that

$$
d^{V} \nabla J=d^{H} J=-\frac{1}{2} d^{H} f \wedge I .
$$

The defining relation (3.50) for $U$ then easily leads to (3.57) and can in fact also be rewritten as

$$
g(U X, Y)=-\frac{1}{2} d^{H} f \wedge \theta_{\ell}(X, Y) .
$$

The final requirement that $\nabla U$ should be equal to $J \Phi-\Phi J$ leads immediately, from (3.57), to (3.58), or can equivalently, from (3.61), be expressed as

$$
(\Phi J-J \Phi)\lrcorner g=\frac{1}{2} \nabla d^{H} f \wedge \theta_{\ell} .
$$

The point is, however, that this again is not an extra condition, but a consequence of the fundamental condition (3.56). This property is not trivial to recognise. An explicit verification of this fact can only come from an integrability analysis on the partial differential equations satisfied by $J$.

The assumption is that $\nabla J=\frac{1}{2}\left(\mathbf{T} \otimes d^{H} f-X_{f} \otimes \theta_{\ell}\right)$ and since $J$ is basic, $\mathrm{D}_{X}^{V} J=0$ for all $X$. So, the commutator property (3.18) implies that

$$
\left.\mathrm{D}_{X}^{H} J=\frac{1}{2}\left(X \otimes d^{H} f-X_{f} \otimes X\right\lrcorner g\right) .
$$

The next interesting commutator to look at here is $\left[\nabla, \mathrm{D}_{X}^{H}\right]$. Using (3.10) and the fact that $\nabla=\mathrm{D}_{\mathbf{T}}^{H}$ gives us that

$$
\left[\nabla, \mathrm{D}_{X}^{H}\right]=\mathrm{D}_{\nabla X}^{H}+\mathrm{D}_{\mathcal{R}(\mathbf{T}, X)}^{V}+\mu_{\operatorname{Rie}(\mathbf{T}, X)} .
$$

The second term vanishes when acting on $J$ and for the last one we have

$$
\begin{aligned}
\mu_{\operatorname{Rie}(\mathbf{T}, X)} J(Y) & =\operatorname{Rie}(\mathbf{T}, X)(J Y)-J \operatorname{Rie}(\mathbf{T}, X) Y \\
& =-\mathrm{D}_{J Y}^{V} \mathcal{R}(\mathbf{T}, X)+J \mathrm{D}_{Y}^{V} \mathcal{R}(\mathbf{T}, X) .
\end{aligned}
$$


Since $\Phi(X)=\mathcal{R}(\mathbf{T}, X)$ and $\mathrm{D}_{Y}^{V} \mathbf{T}=Y$, this expression reduces to

$$
\mu_{\operatorname{Rie}(\mathbf{T}, X)} J(Y)=-\mathrm{D}_{J Y}^{V} \Phi(X)+\mathcal{R}(J Y, X)+J \mathrm{D}_{Y}^{V} \Phi(X)-J \mathcal{R}(Y, X) .
$$

Further, we have from (3.56) and (3.63) that

$$
\begin{aligned}
{\left[\nabla, \mathrm{D}_{X}^{H}\right] J(Y)=} & \frac{1}{2}\left(\left(\mathrm{D}_{Y}^{H} f\right) \nabla X+\left(\mathrm{D}_{Y}^{H} \nabla f\right) X-g(X, Y) \nabla X_{f}\right. \\
& \left.-g(\nabla X, Y) X_{f}-\left(\mathrm{D}_{X}^{H} \mathrm{D}_{Y}^{H} f-\mathrm{D}_{\mathrm{D}_{X}^{H} Y}^{H} f\right) \mathbf{T}+g(Y, \mathbf{T}) \mathrm{D}_{X}^{H} X_{f}\right)
\end{aligned}
$$

So, the identity (3.64), acting on $J$, implies:

$$
\begin{aligned}
& -\mathrm{D}_{J Y}^{V} \Phi(X)-\mathcal{R}(J Y, X)+J \mathrm{D}_{Y}^{V} \Phi(X)-J \mathcal{R}(Y, X)= \\
& \quad \frac{1}{2}\left(\left(\mathrm{D}_{Y}^{H} \nabla f\right) X-g(X, Y) \nabla X_{f}-\left(\mathrm{D}_{X}^{H} \mathrm{D}_{Y}^{H} f-\mathrm{D}_{\mathrm{D}_{X}^{H} Y}^{H} f\right) \mathbf{T}+g(Y, \mathbf{T}) \mathrm{D}_{X}^{H} X_{f}\right)
\end{aligned}
$$

where we have taken $X$ and $Y$ to be basic for simplicity. From this we further obtain

$$
\begin{aligned}
& g(\Phi(X), J Y)+g\left(\mathrm{D}_{Y}^{V} \Phi(X), J \mathbf{T}\right)-g(\mathcal{R}(Y, X), J \mathbf{T})= \\
& \quad \frac{1}{2}\left(\left(\mathrm{D}_{Y}^{H} \nabla f\right) g(X, \mathbf{T})+\left(\nabla^{2} f\right) g(X, Y)-\left(\mathrm{D}_{X}^{H} \mathrm{D}_{Y}^{H} f-\mathrm{D}_{\mathrm{D}_{X}^{H} Y}^{H} f\right) g(\mathbf{T}, \mathbf{T})\right. \\
& \left.\quad-\left(\mathrm{D}_{X}^{H} \nabla f\right) g(Y, \mathbf{T})\right)
\end{aligned}
$$

because $\Phi(\mathbf{T})=0$ and $g(\mathcal{R}(X, Y), \mathbf{T})=0$ (as a consequence of the Bianchiidentity $\left.\sum g(\mathcal{R}(X, Y), Z)=0\right)$. Now putting $Y=\mathbf{T}$ and taking into account that $\mathrm{D}_{\mathrm{T}}^{V} \Phi=2 \Phi$, we get

$$
g(\Phi(X), J \mathbf{T})=\frac{1}{2}\left(\left(\nabla^{2} f\right) g(X, \mathbf{T})-\left(\mathrm{D}_{X}^{H} \nabla f\right) g(\mathbf{T}, \mathbf{T})\right) .
$$

Computing the $\mathrm{D}_{Y}^{V}$ derivative of this result, it follows that

$$
\begin{aligned}
& g\left(\mathrm{D}_{Y}^{V} \Phi(X), J \mathbf{T}\right)+g(\Phi(X), J Y)= \\
& \quad \frac{1}{2}\left(\left(\mathrm{D}_{Y}^{V} \nabla^{2} f\right) g(X, \mathbf{T})-\left(\mathrm{D}_{Y}^{V} \mathrm{D}_{X}^{H} \nabla f\right) g(\mathbf{T}, \mathbf{T})+\left(\nabla^{2} f\right) g(X, Y)\right) \\
& \quad-\left(\mathrm{D}_{X}^{H} \nabla f\right) g(Y, \mathbf{T})
\end{aligned}
$$

where we have used the commutator relations (3.18), (3.64) and (3.9) to see that $\mathrm{D}_{Y}^{V} \nabla^{2} f=2 \mathrm{D}_{Y}^{H} \nabla f$ and $\mathrm{D}_{Y}^{V} \mathrm{D}_{X}^{H} \nabla f=\mathrm{D}_{X}^{H} \mathrm{D}_{Y}^{H} f-\mathrm{D}_{\mathrm{D}_{X}^{H} Y}^{H} f$. Subtracting the same expression with $X$ and $Y$ interchanged and taking into account that $d^{V} \Phi=3 \mathcal{R}$, we get that

$$
3 g(\mathcal{R}(Y, X), J \mathbf{T})=2\left(\mathrm{D}_{Y}^{H} \nabla f\right) g(X, \mathbf{T})-2\left(\mathrm{D}_{X}^{H} \nabla f\right) g(Y, \mathbf{T})+g([\Phi, J] X, Y) .
$$


Finally, if we make use of (3.66) and (3.67) in the expression (3.65), we find:

$$
g([\Phi, J] X, Y)=\frac{1}{2}\left(\left(\mathrm{D}_{X}^{H} \nabla f\right) g(Y, \mathbf{T})-\left(\mathrm{D}_{Y}^{H} \nabla f\right) g(X, \mathbf{T})\right),
$$

which is precisely (3.58).

The final statement (3.59) about $R$ follows directly from comparison between (3.33-3.34) and (3.37-3.38), knowing that $J=\bar{J}$ and using (3.56) and (3.57), with $\mathbf{T}^{V}=\Delta$.

Note that (3.56) is indeed an intrinsic way of writing the special conformal Killing condition (2.3) with $\alpha=d f$. As a matter of fact, in coordinates condition (3.56) reads

$$
J_{j \mid k}^{i}=\frac{1}{2}\left(\delta_{k}^{i} \frac{\partial f}{\partial q^{j}}+g^{i l} \frac{\partial f}{\partial q^{l}} g_{j k}\right) .
$$

and by lowering an index we get

$$
J_{l j \mid k}=\frac{1}{2}\left(g_{l k} \frac{\partial f}{\partial q^{j}}+g_{j k} \frac{\partial f}{\partial q^{l}}\right),
$$

which is the same as (2.3). 


\section{Chapter 4}

\section{More general recursion operators}

In this chapter we will generalise the construction of the type $(1,1)$ tensor fields $R$ on a tangent bundle introduced in chapter 3 . We will no longer start from a basic tensor field $J$, but from a type $(1,1)$ tensor field along the projection. Again we will be interested in the property of having a vanishing Nijenhuis torsion $N_{R}$. One will see that this problem is here a lot more complex then in the situation discussed in chapter 3 . Another issue we want to investigate, is the one of $R$ being invariant under the given dynamics. In addition to these recursion properties (which are already published in [52]), we will also briefly look at a special case of non-invariance of $R$. This discussion can be found in the last section of this chapter.

In chapter 5 the theory is applied to the case of second order dynamics coming from a Finsler metric.

\subsection{R-tensors associated to a $(1,1)$ tensor field along $\tau$}

In this section we propose a generalisation of equation (3.31), $i_{R(\xi)} d d_{S} \ell=$ $i_{\xi} d d_{J^{c} S} \ell$, to the case in which $J$ is a general type $(1,1)$ tensor field along $\tau$; that is to say, we define the R-tensor associated with such a tensor field (for a given Lagrangian $\ell$ ). The basic problem is to know what to replace $J^{c}$ with on the right-hand side, and we discuss this point first. It turns out that it is not necessary to have a Lagrangian for this purpose: a dynamical vector field is enough.

When $J$ is a type $(1,1)$ tensor field along $\tau$ and $\Gamma$ is a given dynamics, there is a natural lift of $J$ to a tensor field $\mathcal{J}_{\Gamma} J$ on $T Q$, which was extensively discussed in [36]. One of its properties is that it reduces to a complete lift $J^{c}$ when $J$ happens to be basic; so this is the natural candidate for attempting to generalise the definition of an R-tensor field. 
Before proceeding to the discussion of R-tensors we examine some properties of $\mathcal{J}_{\Gamma} J$. One may wonder in the first place to what extent $\mathcal{J}_{\Gamma} J \circ S$ could again provide a kind of alternative almost tangent structure, as $J^{c} \circ S$ does. Now $\mathcal{J}_{\Gamma} J$ can be expressed explicitly as follows:

$$
\mathcal{J}_{\Gamma} J=J^{H}+(\nabla J)^{V} .
$$

Since the image of $(\nabla J)^{V}$ is vertical, only the horizontal part plays a role when composing with $S$, so this is really a question about $J^{H} S$. Moreover, it is clear from the defining relations (3.1), (3.2), that actually $J^{H} S=S J^{H}=$ $J^{V}$. Obviously $\left(J^{V}\right)^{2}=0$, and the image of $J^{V}$ coincides with its kernel provided $J$ is non-singular. So $\mathcal{J}_{\Gamma} \circ \circ S=J^{V}$ is indeed an almost tangent structure. In fact since $J^{V}$ vanishes on vertical vectors, its definition doesn't depend on a choice of horizontal distribution (unlike that of $J^{H}$ ).

The canonical almost tangent structure $S$ is integrable, which is to say that its Nijenhuis torsion vanishes. The Nijenhuis torsion of $J^{V}$ is not always zero, however, as the following result indicates.

Proposition 18. $N_{J^{V}}=0$ if and only if $\mathrm{D}_{J X}^{V} J(Y)-\mathrm{D}_{J Y}^{V} J(X)=0$.

Proof. It is easy to see that $N_{J^{V}}$ gives zero when evaluated on two vertical vector fields or on a horizontal and a vertical one. We further have

$$
\begin{aligned}
N_{J^{V}}\left(X^{H}, Y^{H}\right) & =\left[(J X)^{V},(J Y)^{V}\right]-J^{V}\left(\left[(J X)^{V}, Y^{H}\right]+\left[X^{H},(J Y)^{V}\right]\right) \\
& =\left(\mathrm{D}_{J X}^{V}(J Y)-\mathrm{D}_{J Y}^{V}(J X)\right)^{V}-J^{V}\left(\left(\mathrm{D}_{J X}^{V} Y\right)^{H}-\left(\mathrm{D}_{J Y}^{V} X\right)^{H}\right) \\
& =\left(\mathrm{D}_{J X}^{V} J(Y)-\mathrm{D}_{J Y}^{V} J(X)\right)^{V}
\end{aligned}
$$

from which the result follows.

A related question is whether the derivations $d_{S}$ and $d_{J^{V}}$ commute, for which the condition is that the Nijenhuis bracket $\left[J^{V}, S\right]$ vanishes.

Proposition 19. $\left[J^{V}, S\right]=0$ if and only if $\mathrm{D}_{X}^{V} J(Y)-\mathrm{D}_{Y}^{V} J(X)=0$ (i.e. $\left.d^{V} J=0\right)$.

Proof. The proof is a simple computation, completely similar to the one above.

It is useful at this point to introduce certain tensor fields along the projection $\tau$ related to Nijenhuis torsion, which will become important in what follows 
(and played a relevant role already in the study of decoupling of second-order equations [38]). For a general type $(1,1)$ tensor $W$ along $\tau$ we put

$$
\begin{aligned}
& N_{W}^{V}(X, Y)=\mathrm{D}_{W X}^{V} W(Y)-\left(W \mathrm{D}_{X}^{V} W\right)(Y), \\
& N_{W}^{H}(X, Y)=\mathrm{D}_{W X}^{H} W(Y)-\left(W \mathrm{D}_{X}^{H} W\right)(Y),
\end{aligned}
$$

which are called respectively the vertical and horizontal covariant Nijenhuis tensor. Their skew-symmetric parts are denoted by

$$
\mathcal{N}_{W}^{V}(X, Y)=N_{W}^{V}(X, Y)-N_{W}^{V}(Y, X)
$$

and $\mathcal{N}_{W}^{H}$. It is a simple computation to verify that

$$
\mathrm{D}_{W X}^{V} W(Y)-\mathrm{D}_{W Y}^{V} W(X)=\mathcal{N}_{W}^{V}(X, Y)+W\left(d^{V} W(X, Y)\right),
$$

so that the following corollary can be drawn from propositions 18 and 19.

Corollary 1. The derivations $d_{S}$ and $d_{J^{V}}$ constitute a bi-differential calculus if and only if $\mathcal{N}_{J}^{V}=0$ and $d^{V} J=0$.

Proof. We know that $d_{S}^{2}=0$ and the requirements $d_{J}{ }^{2}=0$ and $\left[d_{S}, d_{J^{V}}\right]=0$ are equivalent to the conditions of the two preceding propositions. It is further a simple computation to verify that

$$
\mathrm{D}_{J X}^{V} J(Y)-\mathrm{D}_{J Y}^{V} J(X)=\mathcal{N}_{J}^{V}(X, Y)+J\left(d^{V} J(X, Y)\right) .
$$

The result then readily follows.

Let us come now to the consideration of R-tensors in this context. Take $\Gamma$ to be a (regular) Lagrangian system, so that we have a symplectic form $\omega_{\ell}=d d_{S} \ell$ at our disposal, let $J$ be a type $(1,1)$ tensor along $\tau$, and consider the type $(1,1)$ tensor $R$ on $T Q$ defined by

$$
i_{R(\xi)} d d_{S} \ell=i_{\xi} d d_{J^{V}} \ell, \quad \forall \xi \in \mathcal{X}(T Q) .
$$

In view of what precedes, it is clear that we cannot expect this generalised R-tensor to have all the properties we discussed in section 3.2 for a basic $J$. We first set out to characterise $R$ through its action on horizontal and vertical lifts. We pointed out in section 3.1 that the Poincaré-Cartan 1-form $\theta_{\ell}=d_{S} \ell$ can be written as $\theta_{\ell}=d^{V} \ell$. Similarly, we have that $d_{J V} \ell=$ $J^{V}(d \ell)=J^{H} \theta_{\ell}$ is semi-basic, so that the same 1-form, regarded as a form along $\tau$, can equally be written as $J \theta_{\ell}$. 
Lemma 6. The closed 2-form $\omega_{1}=d d_{J^{V}} \ell$ is characterised by $\omega_{1}\left(X^{V}, Y^{V}\right)=$ 0 , and

$$
\begin{aligned}
& \omega_{1}\left(X^{V}, Y^{H}\right)=\mathrm{D}_{X}^{V}\left(J \theta_{\ell}\right)(Y), \\
& \omega_{1}\left(X^{H}, Y^{H}\right)=d^{H}\left(J \theta_{\ell}\right)(X, Y) .
\end{aligned}
$$

Proof. We have

$$
\begin{aligned}
& \omega_{1}\left(X^{H}, Y^{H}\right) \\
& \quad=\mathcal{L}_{X^{H}}\left(\theta_{\ell}\left((J Y)^{H}\right)\right)-\mathcal{L}_{Y^{H}}\left(\theta_{\ell}\left((J X)^{H}\right)\right)-\theta_{\ell}\left(J^{H}\left(\left[X^{H}, Y^{H}\right]\right)\right) \\
& \quad=\mathrm{D}_{X}^{H}\left(J \theta_{\ell}(Y)\right)-\mathrm{D}_{Y}^{H}\left(J \theta_{\ell}(X)\right)-\theta_{\ell}\left(J\left(\mathrm{D}_{X}^{H} Y-\mathrm{D}_{Y}^{H} X\right)\right) \\
& \quad=\mathrm{D}_{X}^{H}\left(J \theta_{\ell}\right)(Y)-\mathrm{D}_{Y}^{H}\left(J \theta_{\ell}\right)(X)=d^{H}\left(J \theta_{\ell}\right)(X, Y)
\end{aligned}
$$

and

$$
\begin{aligned}
\omega_{1}\left(X^{V}, Y^{H}\right) & =\mathcal{L}_{X^{V}}\left(\theta_{\ell}\left((J Y)^{H}\right)\right)-\theta_{\ell}\left(J^{H}\left(\left[X^{V}, Y^{H}\right]\right)\right) \\
& =\mathrm{D}_{X}^{V}\left(J \theta_{\ell}(Y)\right)-\theta_{\ell}\left(J\left(\mathrm{D}_{X}^{V} Y\right)\right) \\
& =\mathrm{D}_{X}^{V}\left(J \theta_{\ell}\right)(Y),
\end{aligned}
$$

which gives the desired result.

The generalisation of proposition 9, becomes here:

Proposition 20. For a given type $(1,1)$ tensor field $J$ along $\tau$, let $K$ and $U$ be defined by

$$
\begin{aligned}
g(K X, Y) & =\mathrm{D}_{Y}^{V}\left(J \theta_{\ell}\right)(X), \\
g(U X, Y) & =d^{H}\left(J \theta_{\ell}\right)(X, Y) .
\end{aligned}
$$

Then the type $(1,1)$ tensor field $R$ on $T Q$ defined by (4.4) is characterised by

$$
\begin{aligned}
R\left(X^{V}\right) & =(\bar{K} X)^{V} \\
R\left(X^{H}\right) & =(K X)^{H}+(U X)^{V} .
\end{aligned}
$$

Proof. Observe that $\omega_{\ell}\left(R\left(X^{V}\right), Y^{V}\right)=0$, while in view of the definition of $K, U$ and $\bar{K}$, and the defining relation (4.4), and using the results of the above lemma, we can write

$$
\begin{aligned}
& \omega_{\ell}\left(R\left(X^{V}\right), Y^{H}\right)=g(\bar{K} X, Y), \\
& \omega_{\ell}\left(R\left(X^{H}\right), Y^{V}\right)=-g(K X, Y), \\
& \omega_{\ell}\left(R\left(X^{H}\right), Y^{H}\right)=g(U X, Y) .
\end{aligned}
$$


The result now follows from the characterising properties of $\omega_{\ell}$ such as (3.20).

Note that also in this generalised case it follows from the skew-symmetry of the right-hand side in (4.6) that $\bar{U}=-U$. Remark also that $R=K^{H}$ if and only if $K$ is symmetric and $U=0$.

We will need properties of covariant derivatives of $K$ and $U$. These will follow directly from their defining relations by making use of the commutator relations (3.8), (3.9) and (3.10).

Proposition 21. We have, for arbitrary $X, Y, Z \in \mathcal{X}(\tau)$,

$$
\begin{aligned}
& \quad g\left(\mathrm{D}_{Z}^{V} K(X), Y\right)-g\left(\mathrm{D}_{Y}^{V} K(X), Z\right)=0, \\
& \mathrm{D}_{Z}^{V} g(U X, Y)+g\left(\mathrm{D}_{Z}^{V} U(X), Y\right) \\
& \quad=\mathrm{D}_{Z}^{H} g(K Y, X)-\mathrm{D}_{Z}^{H} g(K X, Y)+g\left(d^{H} K(X, Y), Z\right), \\
& \sum_{X, Y, Z}\left(\mathrm{D}_{X}^{H} g(U Y, Z)+g\left(\mathrm{D}_{X}^{H} U(Y), Z\right)\right)=\sum_{X, Y, Z} g(K Z, \mathcal{R}(X, Y)),
\end{aligned}
$$

where $\sum_{X, Y, Z}$ refers to a cyclic sum over the indicated arguments. Furthermore,

$$
\bar{K}=K \quad \Leftrightarrow \quad d^{V}\left(J \theta_{L}\right)=0 .
$$

Proof. The first property follows immediately from taking a vertical derivative of the defining relation (4.5) and making use of the 'vertical Helmholtz property' $\mathrm{D}_{Z}^{V} g(X, Y)=\mathrm{D}_{Y}^{V} g(X, Z)$ and the commutator identity (3.8). For the next two properties, the computations start similarly from the defining relation (4.6) of $U$. Taking a $\mathrm{D}^{V}$ derivative and using the commutator relation (3.9) we find:

$$
\begin{aligned}
\mathrm{D}_{Z}^{V} g(U X, Y)+g\left(\mathrm{D}_{Z}^{V} U(X), Y\right) \\
=\mathrm{D}_{X}^{H} \mathrm{D}_{Z}^{V} J \theta_{\ell}(Y)-\mathrm{D}_{\mathrm{D}_{X}^{H} Z}^{V} J \theta_{\ell}(Y)+\mu_{B(Z, X)} J \theta_{\ell}(Y) \\
\quad-\mathrm{D}_{Y}^{H} \mathrm{D}_{Z}^{V} J \theta_{\ell}(X)+\mathrm{D}_{\mathrm{D}_{Y}^{H} Z}^{V} J \theta_{\ell}(X)-\mu_{B(Z, Y)} J \theta_{\ell}(X) \\
=\mathrm{D}_{X}^{H} g(K Y, Z)-\mathrm{D}_{Y}^{H} g(K X, Z)+g\left(d^{H} K(X, Y), Z\right)
\end{aligned}
$$

The terms involving the tensor $B$ cancel out in view of its full symmetry. So, we get (4.10). For the $\mathrm{D}^{H}$ derivative of (4.6), the computation is somewhat more involved: one has to apply the commutator (3.10) a second time after exploiting the skew-symmetry of $U$, in such a way that a cyclic sum 
combination appears:

$$
\begin{aligned}
\mathrm{D}_{X}^{H}(g(Z, U Y))-\mathrm{D}_{Y}^{H}(g(Z, U X)) \\
=\quad \mathrm{D}_{X}^{H}\left(d^{H} J \theta_{\ell}(Y, Z)\right)-\mathrm{D}_{Y}^{H}\left(d^{H} J \theta_{\ell}(X, Z)\right) \\
=\quad \mathrm{D}_{Y}^{H} \mathrm{D}_{X}^{H} J \theta_{\ell}(Z)-\mathrm{D}_{Z}^{H} \mathrm{D}_{X}^{H} J \theta_{\ell}(Y)-\mathrm{D}_{Y}^{H} \mathrm{D}_{X}^{H} J \theta_{\ell}(Z)+\mathrm{D}_{Z}^{H} \mathrm{D}_{Y}^{H} J \theta_{\ell}(X) \\
\quad+g\left(\mathrm{D}_{X}^{H} Z, U Y\right)-g\left(\mathrm{D}_{Y}^{H} Z, U X\right)+g\left(Z, U \mathrm{D}_{X}^{H} Y-U \mathrm{D}_{Y}^{H} X\right) \\
\quad+\mathrm{D}_{\mathrm{D}_{Z}^{H} X}^{H} J \theta_{\ell}(Y)-\mathrm{D}_{\mathrm{D}_{Z}^{H} Y}^{H} J \theta_{\ell}(X) \\
\quad+\sum_{X, Y, Z} \mathrm{D}_{\mathcal{R}(X, Y)}^{V} J \theta_{\ell}(Z)+\sum_{X, Y, Z} \mu_{\operatorname{Rie}(X, Y)} J \theta_{\ell}(Z) \\
=\quad \mathrm{D}_{Z}^{H}(g(X, U Y))-g\left(\mathrm{D}_{Z}^{H} X, U Y\right)-g\left(X, U \mathrm{D}_{Z}^{H} Y\right)+g\left(\mathrm{D}_{X}^{H} Z, U Y\right) \\
\quad-g\left(\mathrm{D}_{Y}^{H} Z, U X\right)+g\left(Z, U \mathrm{D}_{X}^{H} Y-U \mathrm{D}_{Y}^{H} X\right)+\sum_{X, Y, Z} g(K Z, \mathcal{R}(X, Y))
\end{aligned}
$$

The terms involving the tensor field Rie cancel out in view of the Bianchi identity $\sum \operatorname{Rie}(X, Y) Z=0$ and (4.11) follows. Finally, the characterisation of symmetry of $K$ follows directly from the defining relation.

There are a couple of further consequences which are worth mentioning: one tells us what the obstruction is for $K$ to be symmetric with respect to $\mathrm{D}_{X}^{H} g$; the other shows under what circumstances a property like (4.9) also holds for the horizontal derivatives of $K$.

Corollary 2. For all $X, Y, Z \in \mathcal{X}(\tau)$, we have

$$
\begin{aligned}
& \mathrm{D}_{X}^{H} g(K Y, Z)-\mathrm{D}_{X}^{H} g(K Z, Y)=g\left(\mathrm{D}_{Z}^{V} U(X), Y\right) \\
& \quad-g\left(\mathrm{D}_{Y}^{V} U(X), Z\right)+g\left(d^{H} K(X, Z), Y\right)-g\left(d^{H} K(X, Y), Z\right), \\
& \quad g\left(\mathrm{D}_{Z}^{H} K(X), Y\right)-g\left(\mathrm{D}_{Y}^{H} K(X), Z\right)= \\
& \quad g\left(\mathrm{D}_{Z}^{V} \nabla K(X), Y\right)-g\left(\mathrm{D}_{Y}^{V} \nabla K(X), Z\right) .
\end{aligned}
$$

Proof. If we take (4.10), interchange $Y$ and $Z$ and subtract, the vertical derivatives of $g$ cancel out in view of their Helmholtz property and (4.13) follows. On the other hand, acting with $\nabla$ on (4.10), using $\nabla g=0$ and the by now familiar commutator of $\nabla$ and $\mathrm{D}^{V}$ easily gives (4.14).

In coordinates, using the local basis $\left\{H_{i}, V_{i}\right\}$ of vector fields and its dual $\left\{d q^{i}, \eta^{i}=d u^{i}+\Gamma_{k}^{i} d q^{k}\right\}$, we have

$$
R=K_{j}^{i} H_{i} \otimes d q^{j}+\bar{K}_{j}^{i} V_{i} \otimes \eta^{j}+U_{j}^{i} V_{i} \otimes d q^{j},
$$


where, denoting $V_{i}(\ell)$ for shorthand by $p_{i}$,

$$
\begin{aligned}
K_{j}^{i} & =g^{i k} V_{k}\left(J_{j}^{l} p_{l}\right), \\
U_{j}^{i} & =g^{i k}\left[H_{j}\left(J_{k}^{l} p_{l}\right)-H_{k}\left(J_{j}^{l} p_{l}\right)\right] .
\end{aligned}
$$

It is evident that $K$ and $U$ do not determine $J$ uniquely, or in other words that different $J_{\mathrm{S}}$ may give the same $R$. We shall have occasion to take advantage of this freedom in the choice of $J$ in the next chapter.

One could choose to use the momenta $p_{j}$ as coordinates on $T Q$ rather than the velocities $u^{i}$. Then $g^{i k} V_{k}=\partial / \partial p_{i}$, and the expression for $K$ becomes

$$
K_{j}^{i}=\frac{\partial}{\partial p_{i}}\left(J_{j}^{l} p_{l}\right) .
$$

It is apparent from this equation that $K_{j}^{i}=J_{j}^{i}$ when $J_{j}^{i}$ is independent of the fibre coordinates.

Symmetry properties with respect to $g$ of course refer to the type $(0,2)$ rather than the type $(1,1)$ representation of the tensor under consideration; that is to say, if we put $K_{i j}=g_{i l} K_{j}^{l}$, then

$$
K_{i j}=\frac{\partial}{\partial u^{i}}\left(J_{j}^{l} p_{l}\right)
$$

and the condition (4.12) for symmetry of $K$ is self-evident. Equally evident then is the property

$$
\frac{\partial K_{i j}}{\partial u^{l}}=\frac{\partial K_{l j}}{\partial u^{i}}
$$

which is a coordinate form of (4.9). remark that these relations express that $K$ is the Hessian of a certain function.

The role of $J_{j}^{l} p_{l}$ in the full expression for $R$ has by now become prominent, and this suggests that we should seek to generalise also the notion of complete lift to the cotangent bundle $T^{*} Q$ of a type $(1,1)$ tensor field $J$ along the cotangent bundle projection $\pi: T^{*} Q \rightarrow Q$. Such a $J$ can act on semibasic 1-forms on $T^{*} Q$, regarded as 1-forms along $\pi$, and the canonical 1-form $\theta=p_{i} d q^{i}$ is one of those: then $J \theta=J_{j}^{l} p_{l} d q^{j}$.

Definition 22. Let $J$ be a type $(1,1)$ tensor field along $\pi: T^{*} Q \rightarrow Q$, then the complete lift $\widetilde{J}$ is the $(1,1)$ tensor on $T^{*} Q$ defined by

$$
i_{\widetilde{J}(\xi)} d \theta=i_{\xi} d(J \theta), \quad \forall \xi \in \mathcal{X}\left(T^{*} Q\right) .
$$


Remark: just as with the standard lifting procedures from $Q$ to $T^{*} Q$, one can also define the vertical lift of a $J$ along $\pi$, as being the vector field

$$
J^{v}=J_{j}^{i} p_{i} \frac{\partial}{\partial p_{j}} \in \mathcal{X}\left(T^{*} Q\right) .
$$

The right-hand side in the defining relation (4.21) can then be written as $i_{\xi} \mathcal{L}_{J^{v}} d \theta=i_{\xi} d i_{J^{v}} d \theta$.

The fact that on $T Q$ the R-tensor of $J$ is a more interesting tensor field to look at than either the generalised complete lift $\mathcal{J}_{\Gamma} J$ or the horizontal lift $J^{H}$ is now underscored by the following generalisation of proposition 7 in chapter 3 .

Proposition 22. Let Leg : $T Q \rightarrow T^{*} Q$ denote the Legendre transform defined by the given regular Lagrangian $\ell$, then $\operatorname{Leg}_{*} R=\widetilde{J}$.

Proof. As a preliminary remark, starting from a $J$ along $\tau$, we are using the same notation for the corresponding $J$ along $\pi$, which is in effect $\operatorname{Leg}_{*} J$ and is obtained by simply expressing the components $J_{j}^{i}(q, u)$ in terms of the cotangent bundle coordinates $(q, p)$. Now, as observed before, the 2-form $\omega_{1}=d d_{J^{V}} \ell$ in the right-hand side of (4.4), if we identify semi-basic forms with forms along $\tau$, can be written with a slight abuse of notation as $d\left(J \theta_{\ell}\right)$, and it is clear then that its image under $\operatorname{Leg}_{*}$ is just $d(J \theta)$. The statement now immediately follows.

From the coordinate expression (4.15) of $R$ and the comment (4.18) about $K$, one can in fact immediately surmise that $\widetilde{J}$ must have the form

$\widetilde{J}=\frac{\partial}{\partial p_{i}}\left(J_{j}^{l} p_{l}\right)\left(X_{i} \otimes d q^{j}+\frac{\partial}{\partial p_{j}} \otimes \pi_{i}\right)+\left(X_{k}\left(J_{j}^{l} p_{l}\right)-X_{j}\left(J_{k}^{l} p_{l}\right)\right) \frac{\partial}{\partial p_{j}} \otimes d q^{k}$,

where $X_{k}=\operatorname{Leg}_{*} H_{k}$ and $\operatorname{Leg}_{*} \eta^{j}=g^{j k} \pi_{k}$. For completeness, one can verify that

$$
X_{k}=\frac{\partial}{\partial q^{k}}-\widetilde{\Gamma}_{l k} \frac{\partial}{\partial p_{l}}, \quad \text { with } \quad \widetilde{\Gamma}_{l k}=g_{l j}\left(\Gamma_{k}^{j}+\frac{\partial^{2} H}{\partial p_{j} \partial q^{k}}\right),
$$

where $H$ is the Hamiltonian corresponding to $\ell$. Correspondingly, $\pi_{k}=$ $d p_{k}+\widetilde{\Gamma}_{k l} d q^{l}$. It is worthwhile observing that $\widetilde{\Gamma}_{l k}=\widetilde{\Gamma}_{k l}$. In fact, one can easily compute from the definition of the connection coefficients $\Gamma_{j}^{i}$ that a tangent bundle expression for the $\widetilde{\Gamma}_{l k}$ can be written

$$
\widetilde{\Gamma}_{l k}=\frac{1}{2}\left(\Gamma\left(g_{l k}\right)-\frac{\partial^{2} \ell}{\partial u^{k} \partial q^{l}}-\frac{\partial^{2} \ell}{\partial u^{l} \partial q^{k}}\right),
$$


which is manifestly symmetric.

Before embarking on the two aspects of recursion now, let us state for later use two more properties of $R$ with respect to the tangent bundle structure on $T Q$ (as encoded by the tensor $S$ ).

Proposition 23. $R S=S R \quad \Leftrightarrow \quad K=\bar{K}$.

Proof. This is a trivial observation from the structure of $R$.

Concerning the Nijenhuis bracket of $R$ and $S$, one can verify easily that $[R, S]\left(X^{V}, Y^{V}\right)=0$, while

$$
\begin{aligned}
& {[R, S]\left(X^{V}, Y^{H}\right)=-\left(\mathrm{D}_{Y}^{V} \bar{K}(X)+\mathrm{D}_{X}^{V} K(Y)\right)^{V},} \\
& {[R, S]\left(X^{H}, Y^{H}\right)=\left(d^{V} K(X, Y)\right)^{H}+\left(\left(d^{V} U-d^{H} K\right)(X, Y)\right)^{V} .}
\end{aligned}
$$

It looks as though it would be much too strong a condition to expect that $[R, S]$ could be zero, but the following weaker requirement will be useful further on and follows immediately from these relations.

Proposition 24. $[R, S]$ is a vertical-vector-valued 2-form if and only if $d^{V} K=0$.

\subsection{Invariant $\mathrm{R}$-tensors}

We now turn again to the issue of $R$ being a recursion tensor in the sense of being a symmetry generator for $\Gamma$, i.e. we investigate the properties of R-tensors which have vanishing Lie-derivative with respect to $\Gamma$.

Theorem 14. Let $R$ be a type $(1,1)$ tensor on $T Q$ defined by tensor fields $K$ and $U$ along $\tau$ as in equations (4.5), (4.6). Then $\mathcal{L}_{\Gamma} R=0$ if and only if: $K$ is symmetric with respect to $g, U=0, K$ has vanishing dynamical covariant derivative $\nabla K$ and commutes with the Jacobi endomorphism $\Phi$.

Proof. Using the characterisation (4.7) and (4.8) of $R$ and the bracket relations (3.13), it is straightforward to verify that

$$
\begin{aligned}
& \mathcal{L}_{\Gamma} R\left(X^{V}\right)=(K-\bar{K})(X)^{H}+(\nabla \bar{K}+U)(X)^{V}, \\
& \mathcal{L}_{\Gamma} R\left(X^{H}\right)=(\nabla K-U)(X)^{H}+(\Phi K-\bar{K} \Phi+\nabla U)(X)^{V} .
\end{aligned}
$$

Expressing that the horizontal and vertical parts must vanish separately, the result now immediately follows. 
Note that the only change here with respect to the result for basic $J$ in chapter 3 is that $J$ is replaced by $K$. Notice also that since $U$ must be zero, invariant R-tensors are of the form $R=K^{H}$, where $K$ satisfies the conditions of the theorem.

\subsubsection{Alternative Lagrangians}

The theory about the existence of alternative Lagrangians of the same second-order differential equations (not just by addition of a total time derivative or multiplication by a constant) is already extensively examined (see for example [11], [19] and [20]). It is known that one can associate to a pair of alternative Lagrangians $\ell$ and $\ell^{\prime}$ of a dynamical system $\Gamma$, a type $(1,1)$ tensor field $T$, uniquely defined by

$$
\omega_{\ell}(T X, Y)=\omega_{\ell^{\prime}}(X, Y) \quad \forall X, Y \in \mathcal{T} \mathcal{Q} .
$$

The tensor field $T$ then has the following properties:

- $\mathcal{L}_{\Gamma} T=0$

- $\omega_{\ell}(T X, Y)=\omega_{\ell}(X, T Y)$;

- $T \circ S=S \circ T$.

Conversely, if a type $(1,1)$ tensor field $T$ on $T Q$, is invariant, symmetric with respect to $\omega_{\ell}$ and commutes with $S$, it will give rise to a different Lagrangian $\ell^{\prime}$ for the same $\Gamma$, provided that the 2 -form $i_{T} \omega_{\ell}$ is closed.

We shall see that this theory fits entirely within our present framework. To begin with, we prove an economical version of the way alternative Lagrangians arise in the context of R-tensors.

Proposition 25. For a given regular Lagrangian $\ell$ and given type $(1,1)$ tensor $J$ along $\tau$, consider the tensor $K$ defined by (4.5). Assume $K$ is symmetric, commutes with $\Phi$ and satisfies $\nabla K=0$, and put $\left.g^{\prime}=K\right\lrcorner g$. Then $g^{\prime}$ satisfies the Helmholtz conditions (3.17) and hence, provided that $K$ is non-singular, defines an alternative Lagrangian for $\Gamma$.

Proof. Symmetry of $K$ means the same as saying that $g^{\prime}$ is symmetric, while the commutativity of $K$ and $\Phi$ then implies that also $\Phi\lrcorner g^{\prime}$ is symmetric. $\nabla g^{\prime}=0$ trivially follows from $\nabla g=0$ and $\nabla K=0$. The vertical 
Helmholtz property of $g$, together with (4.9), finally implies that $g^{\prime}$ will have the same property.

Comparing the above statement with theorem 14, it is not immediately obvious why nothing is said about $U$. Let us explain this point more clearly as follows. Starting from a tensor $J$ along $\tau$, the corresponding $R$ with components $K$ and $U$ is uniquely determined. If $K$ satisfies the above requirements, we have an alternative Lagrangian $\ell^{\prime}$, even though the $R$ we started from need not be invariant since $U$ need not be zero. The point is, however, that there is a different tensor then, related to the same $K$, which is invariant, namely $R^{\prime}=K^{H}$. It is the tensor obtained by replacing the $\omega_{1}$ we first thought of in the definition (4.4) by $\omega_{\ell^{\prime}}$.

It is worth explaining that $R^{\prime}$ is also an R-tensor in more detail by the following two arguments: (i) with $K$ as the starting point, we discuss what is needed to have that $K$ is derived from a $J$ in such a way that the corresponding $U$ is zero; (ii) we show how an alternative $\ell^{\prime}$ gives rise to such a $K$.

Suppose that the tensor $K$ is symmetric with respect to $g$ and satisfies (4.9). The latter means (see e.g. (4.20)) that the covariant form of $K$ comes from some 1-form $\beta$, in the sense that $K=\mathrm{D}^{V} \beta$. The symmetry of $K$ further implies that $\beta=\mathrm{D}^{V} F$ for some function $F$, so that $K$ is a Hessian. Having fixed a $\beta$, we can clearly find a tensor $J$, indeed many tensors $J$, such that $J_{i}^{s} p_{s}=\beta_{i}$, but the corresponding $U$ does not depend on the freedom in $J$. The 1-form $\beta$ itself is determined in the first stage to within an arbitrary 1 -form $\beta_{0}$ on the base manifold $Q$. Assume next that $\nabla K=0$. Then the property (4.14) says that $K_{i j \mid l}=K_{l j \mid i}$, where $K_{i j}=V_{i}\left(\beta_{j}\right)=V_{j}\left(\beta_{i}\right)$, or explicitly

$$
H_{l}\left(V_{j}\left(\beta_{i}\right)\right)-\Gamma_{l j}^{s} V_{s}\left(\beta_{i}\right)=H_{i}\left(V_{j}\left(\beta_{l}\right)\right)-\Gamma_{i j}^{s} V_{s}\left(\beta_{l}\right) .
$$

Interchanging the horizontal and vertical derivatives, it then follows that $V_{j}\left(H_{l}\left(\beta_{i}\right)-H_{i}\left(\beta_{l}\right)\right)=0$. Hence $H_{l}\left(\beta_{i}\right)-H_{i}\left(\beta_{l}\right)$ are the components of a basic 2 -form and thus the freedom of selecting a basic $\beta_{0}$ can be used to cancel them, i.e. set $J_{i}^{s} p_{s}=\beta_{i}-\beta_{0 i}$ with $d^{H} \beta=d \beta_{0}$. This means that the corresponding $U$ then is zero in view of (4.17). In conclusion, starting from a tensor $K$, the property (4.9) ensures that $K$ comes from some $J$, and if $K$ is symmetric and $\nabla$-parallel, it can always be arranged that the corresponding $U$ is zero. Concerning point (ii) now, if $g^{\prime}$ is the metric tensor along $\tau$ determined by the alternative Lagrangian $\ell^{\prime}$ (assumed regular), and we define $K$ by $\left.g^{\prime}=K\right\lrcorner g$, then $K$ is symmetric and satisfies (4.9) and 
$\nabla K=0$, as a result of the Helmholtz conditions satisfied by both $g$ and $g^{\prime}$. Hence, it comes from a $J$ with $U=0$ and $K^{H}$ is an R-tensor.

The best known application of invariant tensors is the generation of first integrals. In that field, a bi-differential calculus can play a relevant role, and it is worth trying to understand in detail what the distinctive role in this application is of invariance of $R$.

The equation $\mathcal{L}_{\Gamma} R=0$, or essentially $\nabla K=0$, is a Lax-type equation (for a general geometric approach to Lax equations, see [6]). It follows that the trace of $R$ (and of all its powers) is a first integral of $\Gamma$. In the context of alternative Lagrangians, this result is often referred to as the Hojman-Harleston theorem [22]. So, the equation $\mathcal{L}_{\Gamma} R=0$ is related with the theory of integrable systems and this brings us to the other recursion aspect, namely the study of the Nijenhuis tensor of $R$. This enters the scene when one wishes the integrals to be in involution. In fact, it was shown in [11], still in the context of alternative Lagrangians but translated to our present set-up, that if the eigenfunctions of $R$ have multiplicity 2 and $N_{R}=0$ then these eigenfunctions form an involutive set of first integrals. Since the tensors $R$ we consider, are of the form $K^{H}$, the eigenvalues of $R$ have even degeneracy. So, if $K$ has distinct eigenvalues at each point, then the eigenvalues of $R$ have double degeneracy. The computation of $N_{R}$ in all generality, i.e. without linking it to invariance properties of $R$, is quite tedious and will be addressed in the next section. But for the subclass of horizontal lifts of an arbitrary $(1,1)$ tensor $K$ along $\tau$, which is the situation we encounter here, things are a lot simpler, so we may discuss them already now. Indeed, as was mentioned in [38], we have

$$
\begin{aligned}
& \mathcal{N}_{K^{H}}\left(X^{V}, Y^{V}\right)=\mathcal{N}_{K}^{V}(X, Y)^{V}, \\
& \mathcal{N}_{K^{H}}\left(X^{H}, Y^{V}\right)=N_{K}^{H}(X, Y)^{V}-N_{K}^{V}(Y, X)^{H}, \\
& \mathcal{N}_{K^{H}}\left(X^{H}, Y^{H}\right)=\mathcal{N}_{K}^{H}(X, Y)^{H}+\mathcal{R}_{K}(X, Y)^{V},
\end{aligned}
$$

where the term related to the curvature $\mathcal{R}$ is defined by

$$
\mathcal{R}_{K}(X, Y)=\mathcal{R}(K X, K Y)-K(\mathcal{R}(K X, Y)+\mathcal{R}(X, K Y))+K^{2}(\mathcal{R}(X, Y)) .
$$

So vanishing of $N_{K^{H}}$ reduces to three conditions (not five as one might expect), namely

$$
N_{K}^{V}=0, \quad N_{K}^{H}=0, \quad \mathcal{R}_{K}=0 .
$$

If $K^{H}$ is actually the invariant tensor $R$ of theorem 14, there is a further reduction. 
Proposition 26. Under the conditions of theorem 14, we have $N_{R}=0$ if and only if $N_{K}^{V}=0$.

Proof. It was shown in [38] that in all generality, $\nabla N_{K}^{V}=N_{K, \nabla K}^{V}-N_{K}^{H}$, where

$$
N_{W, T}^{V}(X, Y)=\left(\mathrm{D}_{W X}^{V} T+\mathrm{D}_{T X}^{V} W-T \circ \mathrm{D}_{X}^{V} W-W \circ \mathrm{D}_{X}^{V} T\right)(Y)
$$

Because we know that $\nabla K=0$ in this situation, it follows that $N_{K, \nabla K}^{V}=0$. Thus $N_{K}^{V}=0$ will imply $N_{K}^{H}=0$. Also derived in [38] is an identity which expresses $\mathcal{R}_{K}$ as a sum of terms, each of which involves either $N_{K}^{V}$ or $\Phi K-K \Phi:$

$$
\begin{aligned}
3 R_{K}(X, Y)= & N_{K}^{V}(X, \Phi Y)-N_{K}^{V}(Y, \Phi X)-\Phi N_{K}^{V}(X, Y)+\Phi N_{K}^{V}(Y, X) \\
& +\left(\mathrm{D}_{K X}^{V}[\Phi, K]\right) Y-\left(K \circ \mathrm{D}_{X}^{V}[\Phi, K]\right) Y \\
& -\left(\mathrm{D}_{K Y}^{V}[\Phi, K]\right) X-\left(K \circ \mathrm{D}_{Y}^{V}[\Phi, K]\right) X-[\Phi, K] d^{V} K(X, Y) .
\end{aligned}
$$

Hence, under the present assumptions, $\mathcal{R}_{K}$ will automatically be zero as well.

As seen in subsection 1.2.2 the bi-Hamiltonian description which arises from a Poisson-Nijenhuis structure is related to the issue of integrable systems. Similarly as in the case of a basic tensor $J$ (see chapter 3 ), we have here a Poisson-Nijenhuis structure if we assume that the Nijenhuis torsion of $R$ is zero. Indeed, the fact that the 2 -form $\omega_{1}$ on the right-hand side of (4.4) is closed, implies that we have a second Poisson structure and the vanishing of the Nijenhuis tensor makes the two Poisson structures compatible. In subsection 1.2.2, we have seen that if we have a Poisson-Nijenhuis structure, it follows, in particular, that

$$
i_{\mathcal{L}_{\Gamma} R} \omega_{\ell}=-2 d d_{R} E_{\ell}
$$

(see (1.9)). Now we come to the subject of bi-differential calculus which is a useful tool for generating functions (not even first integrals, necessarily) which are in involution with respect to both Poisson structures (theorem 2 ). The algorithmic process by which such functions are generated (at least locally) requires an initial function $f$ which satisfies $d d_{R} f=0$. Obviously, when $R$ is invariant, we have such an initial function since (4.29) shows that $d d_{R} E_{\ell}=0$, and the hierarchy of functions in involution will be first integrals. 
Such invariant R-tensors also have an interesting application in the theory of separable Lagrangian system by which one means that there exists a coordinate transformation on $Q$ such that the system decouples into a number of lower dimensional subsystems in those coordinates. Indeed, the type $(1,1)$ tensors we consider here, are getting close to the kind of tensors discussed in $[19,20]$. An extra assumption we have to make is that the tensors must be algebraically diagonisable. The theorem about separability proven in [19], or better, the slightly corrected version of this theorem as given in [38], reads as follows:

Theorem 15. Let $\Gamma$ be a SODE and $T$ be a diagonisable tensor field on $T Q$ with doubly degenerate eigenvalues. Assume further that $\mathcal{L}_{\Gamma} T=0, N_{T}=0$ and that the bracket of $S$ and $T$ takes vertical values. Then $\Gamma$ is separable.

So, for the present context, we can state the following result.

Proposition 27. Suppose that $\mathcal{L}_{\Gamma} R=0$ and that $K$ further has the properties $N_{K}^{V}=0$ and $d^{V} K=0$. Then, if $K$ is diagonalisable, the given system $\Gamma$ is separable.

Proof. We know that $R$ is invariant, has vanishing Nijenhuis torsion and has doubly degenerate eigenvalues. Moreover, since $K$ is symmetric $R$ commutes with $S$ and since $d^{V} K=0$ the Nijenhuis bracket of $R$ and $S$ takes vertical values (see proposition 24 ). These are exactly the conditions which are required for the above theorem.

\subsection{The Nijenhuis torsion of $\widetilde{J}$ and $R$}

Whenever type $(1,1)$ tensors are part of a theory one is bound to study the effect of vanishing Nijenhuis torsion. Not unexpectedly, here it will be a much more complicated issue then in the case of a basic type $(1,1)$ tensor $J$, but still we will find interesting simplifications occurring in the number of conditions.

First we shall approach the computation of the conditions for vanishing Nijenhuis torsion of $R$ in quite a general way.

Let $\omega$ be a symplectic 2-form on an even dimensional manifold, and $\omega_{1}$ any 2 -form; define the $(1,1)$-tensor $R$ as before by $i_{R(\xi)} \omega=i_{\xi} \omega_{1}$. We shall derive an expression for the Nijenhuis torsion of $R$ in terms of $\omega$ and $R$, under the assumption that $\omega_{1}$ is closed. The exterior derivative $d \omega_{1}$ can also 
be expressed in terms of $\omega$ and $R$; the two expressions have an unexpected affinity. Finally, it will be shown that the condition for the vanishing of the Nijenhuis torsion of $R$, when $\omega_{1}$ is closed, can be written $d_{R} \omega_{1}=0$.

In order to derive the last result we shall need to employ Frölicher-Nijenhuis calculus, and we start by listing some relevant generalities concerning that calculus [21].

It follows from the definition of $R$ that $\omega(R \xi, \eta)=\omega(\xi, R \eta)$, and therefore that $i_{R} \omega=2 \omega_{1}$. Observe, however, that this relation cannot be used to define $R$ directly, because one needs to know that $R$ is symmetric with respect to $\omega$ before the left-hand side fixes $R$ in view of the non-degeneracy of $\omega$. But it easily further follows now that

$$
i_{R} i_{R} \omega=2 i_{R^{2}} \omega=2 i_{R} \omega_{1} .
$$

Assume next that $d \omega_{1}=0$ (as well as $d \omega=0$ ). Then obviously $d i_{R} \omega=0$, from which it follows that also $d_{R} \omega=i_{R} d \omega-d i_{R} \omega=0$, and that $d_{R^{2}} \omega=$ $-d i_{R^{2}} \omega=-d i_{R} \omega_{1}=d_{R} \omega_{1}$.

From relation (1.12), it then follows that

$$
2 d_{R} \omega_{1}=d_{R} i_{R} \omega=2 i_{N_{R}} \omega-d_{R^{2}} \omega,
$$

or finally

$$
2 i_{N_{R}} \omega=3 d_{R} \omega_{1} .
$$

It is clear that $N_{R}=0$ will imply $d_{R} \omega_{1}=0$, but the fact that these conditions are actually equivalent needs a stronger result, because

$$
i_{N_{R}} \omega(\xi, \eta, \zeta)=\sum_{\xi, \eta, \zeta} \omega\left(N_{R}(\xi, \eta), \zeta\right),
$$

Thus (4.30) does not determine $N_{R}$, unless we know, what we will show now, that the three terms in the cyclic sum on the right of (4.31) are actually equal.

Proposition 28. If $R$ is defined by $i_{R(\xi)} \omega=i_{\xi} \omega_{1}$, where $\omega$ is a symplectic 2-form and $\omega_{1}$ any 2-form, then

$$
d \omega_{1}(\xi, \eta, \zeta)=\sum_{\xi, \eta, \zeta} \zeta(\omega(R \xi, \eta))-\sum_{\xi, \eta, \zeta} \omega(R([\xi, \eta]), \zeta) .
$$

If in addition $d \omega_{1}=0$ then

$$
\omega\left(N_{R}(\xi, \eta), \zeta\right)=-\sum_{\xi, \eta, \zeta} \zeta(\omega(R \xi, R \eta))+\sum_{\xi, \eta, \zeta} \omega(R([\xi, \eta]), R \zeta) .
$$

It follows that when $d \omega_{1}=0, N_{R}=0$ if and only if $d_{R} \omega_{1}=0$. 
Proof. The first result follows simply from the identity $d \omega_{1}(\xi, \eta, \zeta)=$ $\sum \xi\left(\omega_{1}(\eta, \zeta)\right)-\sum \omega_{1}([\xi, \eta], \zeta)$ and the defining relation for $R$. To obtain the second result one uses the identity $d \omega(\xi, \eta, \zeta)=\sum \xi(\omega(\eta, \zeta))-\sum \omega([\xi, \eta], \zeta)$ to express in particular the fact that $d \omega(\xi, R \eta, R \zeta)=0$. The two terms $R \eta \omega(R \zeta, \xi)$ and $R \zeta \omega(\xi, R \eta)$ may be each replaced by five other terms, none of which involves a derivative by $R(\cdot)$. Therefore we use expression (4.32) applied on $d \omega_{1}(\xi, R \eta, \zeta)$ and $d \omega_{1}(\xi, \eta, R \zeta)$, assuming that $d \omega_{1}=0$. The resulting expression is

$$
\begin{aligned}
0=d \omega(\xi, R \eta, R \zeta)= & -\sum_{X, Y, Z} \xi \omega(R \eta, R \zeta)+\sum_{X, Y, Z} \omega(R[\xi, \eta], R \zeta) \\
& -\omega\left(R^{2}[\eta, \zeta]-R[R \eta, \zeta]-R[\eta, R \zeta]+[R \eta, R \zeta], \xi\right)
\end{aligned}
$$

from which (4.33) follows. In particular, (4.33) implies that the left-hand side $\omega\left(N_{R}(\xi, \eta), \zeta\right)$ is invariant for cyclic permutations. The final statement now immediately follows from (4.30).

Notice the similarity between the expression for $d \omega_{1}(\xi, \eta, \zeta)$ and the expression for $\omega\left(N_{R}(\xi, \eta), \zeta\right)$ when $d \omega_{1}=0$.

We now obtain explicit expressions for the conditions for the vanishing of the Nijenhuis torsion of $\widetilde{J}$ and $R$, starting with the former.

Now $\widetilde{J}$ is determined by a given $J$ along $\pi$ and the canonical 1-form $\theta$ only, i.e. it does not depend on a given dynamics of Lagrangian or Hamiltonian type. For this reason, there is no advantage to be gained from working in any local frame other than a natural coordinate frame. It is clear from the expression (4.23), or in fact from a direct interpretation of the definition (4.21), that in natural bundle coordinates $\widetilde{J}$ will be of the form

$$
\widetilde{J}=K_{j}^{i}\left(\frac{\partial}{\partial q^{i}} \otimes d q^{j}+\frac{\partial}{\partial p_{j}} \otimes d p_{i}\right)+M_{k j} \frac{\partial}{\partial p_{j}} \otimes d q^{k},
$$

where

$$
K_{j}^{i}=\frac{\partial}{\partial p_{i}}\left(J_{j}^{s} p_{s}\right), \quad M_{k j}=\frac{\partial}{\partial q^{k}}\left(J_{j}^{s} p_{s}\right)-\frac{\partial}{\partial q^{j}}\left(J_{k}^{s} p_{s}\right) .
$$

The following immediate properties of the coefficients of $\widetilde{J}$ will be used below:

$$
\frac{\partial K_{k}^{l}}{\partial p_{j}}=\frac{\partial K_{k}^{j}}{\partial p_{l}}, \quad \frac{\partial M_{j k}}{\partial p_{l}}=\frac{\partial K_{k}^{l}}{\partial q^{j}}-\frac{\partial K_{j}^{l}}{\partial q^{k}}, \quad \sum_{i, j, k} \frac{\partial M_{j k}}{\partial q^{i}}=0,
$$


where $\sum_{i, j, k}$ again refers to a cyclic sum over the indicated indices. In fact, these properties merely express the fact that the 2 -form $d(J \theta)$ in the defining relation of $\widetilde{J}$ is closed; that is, they are the coordinate expressions of the first result of the proposition above in this case. They are also directly related to the three properties of proposition 21 via the Legendre transform.

Theorem 16. The Nijenhuis tensor of $\widetilde{J}$ vanishes if and only if

$$
\begin{aligned}
& A_{k}^{i j}:=K_{l}^{i} \frac{\partial K_{k}^{j}}{\partial p_{l}}-K_{l}^{j} \frac{\partial K_{k}^{i}}{\partial p_{l}}=0, \\
& B_{k j}^{i}:=K_{k}^{l} \frac{\partial K_{j}^{i}}{\partial q^{l}}-K_{j}^{l} \frac{\partial K_{k}^{i}}{\partial q^{l}}+M_{k l} \frac{\partial K_{j}^{i}}{\partial p_{l}}-M_{j l} \frac{\partial K_{k}^{i}}{\partial p_{l}}+K_{l}^{i} \frac{\partial M_{j k}}{\partial p_{l}}=0, \\
& \sum_{i, j, k} C_{i j k}:=\sum_{i, j, k}\left(K_{i}^{l} \frac{\partial M_{j k}}{\partial q^{l}}+M_{i l} \frac{\partial M_{j k}}{\partial p_{l}}\right)=0 .
\end{aligned}
$$

Proof. This can be obtained from proposition 28; alternatively, it can be established by a simple coordinate calculation in which attention must be paid to making appropriate use of the properties (4.36) for recombining the various coefficients in the right format. One obtains

$$
\begin{aligned}
& N_{\widetilde{J}}\left(\frac{\partial}{\partial p_{i}}, \frac{\partial}{\partial p_{j}}\right)=A_{k}^{i j} \frac{\partial}{\partial p_{k}}, \\
& N_{\widetilde{J}}\left(\frac{\partial}{\partial p_{i}}, \frac{\partial}{\partial q^{j}}\right)=A_{j}^{i k} \frac{\partial}{\partial q^{k}}+B_{k j}^{i} \frac{\partial}{\partial p_{k}}, \\
& N_{\widetilde{J}}\left(\frac{\partial}{\partial q^{i}}, \frac{\partial}{\partial q^{j}}\right)=B_{i j}^{k} \frac{\partial}{\partial q^{k}}+\sum_{i, j, k} C_{i j k} \frac{\partial}{\partial p_{k}},
\end{aligned}
$$

which implies the stated result.

The structure of the conditions for the vanishing of $N_{\widetilde{J}}$ is apparent: they are of the same form as the identities (4.36) satisfied by the coefficients of $\widetilde{J}$, but with the coordinate vector fields replaced by their images under $\widetilde{J}$. To be precise, they can be cast in the form

$$
\begin{aligned}
& \widetilde{J}\left(\frac{\partial}{\partial p_{i}}\right)\left(K_{k}^{j}\right)-\widetilde{J}\left(\frac{\partial}{\partial p_{j}}\right)\left(K_{k}^{i}\right)=0, \\
& \widetilde{J}\left(\frac{\partial}{\partial p_{i}}\right)\left(M_{j k}\right)=\widetilde{J}\left(\frac{\partial}{\partial q^{j}}\right)\left(K_{k}^{i}\right)-\widetilde{J}\left(\frac{\partial}{\partial q^{k}}\right)\left(K_{j}^{i}\right), \\
& \sum_{i, j, k} \widetilde{J}\left(\frac{\partial}{\partial q^{i}}\right)\left(M_{j k}\right)=0 .
\end{aligned}
$$


The general observation made after proposition 28 provides the background explanation for this feature.

We now come back to the situation on the tangent bundle, where we have the tools to approach the question in an intrinsic way. So, take $\omega$ now to be the closed 2-form $\omega_{L}=g^{K}$ on $T Q$ and $\omega_{1}=d\left(J \theta_{\ell}\right)$. In principle one should evaluate $N_{R}$ on all combinations of horizontal and vertical lifts and identify each time the horizontal and vertical component of the result; but the cyclic sum invariance of $\omega\left(N_{R}(\xi, \eta), \zeta\right)$ means that, for example, $\omega\left(N_{R}\left(X^{H}, Y^{V}\right), Z^{V}\right)$ will follow from $\omega\left(N_{R}\left(Y^{V}, Z^{V}\right), X^{H}\right)$; furthermore, it is easy to see from the expression in proposition 28 that $\omega\left(N_{R}\left(X^{V}, Y^{V}\right), Z^{V}\right)=$ 0 . Thus in the end only three components need to be computed, which is in agreement with the coordinate results in theorem 16 .

Theorem 17. Let $R$ be defined by $i_{R(\xi)} \omega_{\ell}=i_{\xi} d\left(J \theta_{\ell}\right)$ and thus be characterised as in proposition 20. Then, the necessary and sufficient conditions for $N_{R}$ to vanish are:

$$
\begin{aligned}
& \mathrm{D}_{\bar{K} X}^{V} K(Y)-K\left(\mathrm{D}_{X}^{V} K(Y)\right)=0, \quad \text { or equivalently } \quad \mathcal{N}_{\bar{K}}^{V}=0, \\
& \mathcal{N}_{K}^{H}(X, Y)+\mathrm{D}_{U X}^{V} K(Y)-\mathrm{D}_{U Y}^{V} K(X)=0, \\
& \sum_{X, Y, Z}\left(g\left(d^{H} K(U Y, Z), X\right)+g\left(d^{H} K(Y, U Z), X\right)+g\left(d^{H} K(Y, Z), U X\right)\right) \\
& -\sum_{X, Y, Z}\left(g\left(\mathrm{D}_{Y}^{V} U(U Z), X\right)-g\left(\mathrm{D}_{Z}^{V} U(U Y), X\right)+g\left(d^{H} U(Y, Z), K X\right)\right) \\
& =\sum_{X, Y, Z} g\left(\mathcal{R}(Y, Z), K^{2} X\right) .
\end{aligned}
$$

PROOF. In agreement with what was said above, we need to compute only, for example,

$$
\omega_{\ell}\left(N_{R}\left(X^{V}, Y^{V}\right), Z^{H}\right), \quad \omega_{\ell}\left(N_{R}\left(X^{H}, Y^{V}\right), Z^{H}\right), \quad \omega_{\ell}\left(N_{R}\left(X^{H}, Y^{H}\right), Z^{H}\right) .
$$

Considering the relation (4.33) with $\xi=X^{V}, \eta=Y^{V}, \zeta=Z^{H}$, making use of the defining relations $(4.7,4.8)$ of $R$ and (3.20), the first sum on the right readily reduces to $-\mathrm{D}_{X}^{V}\left(g\left(Y, K^{2} Z\right)\right)+\mathrm{D}_{Y}^{V}\left(g\left(X, K^{2} Z\right)\right)$. In evaluating such expressions, there is no need to take account of terms which involve derivatives of vector field arguments: we know that these will always cancel out in the end since we are computing a tensorial quantity. Terms involving derivatives of $g$ cancel out in view of one of the Helmholtz properties (3.17), there remains:

$$
g\left(X, \mathrm{D}_{Y}^{V} K^{2}(Z)\right)-g\left(Y, \mathrm{D}_{X}^{V} K^{2}(Z)\right)=0
$$


One can easily eliminate $g$ from this expression by making appropriate use of the property $g\left(X \mathrm{D}_{Y}^{V} K(Z)\right)=g\left(Y, \mathrm{D}_{X}^{V} K(Z)\right)$ (see (4.9)), namely

$$
\begin{aligned}
& g\left(X, \mathrm{D}_{Y}^{V} K^{2}(Z)-g\left(Y, \mathrm{D}_{X}^{V} K^{2}(Z)\right)\right. \\
& =g\left(X, \mathrm{D}_{Y}^{V} K(K Z)\right)+g\left(\bar{K} X, \mathrm{D}_{Y}^{V} K(Z)\right)-g\left(Y, \mathrm{D}_{X}^{V} K(K Z)\right)-g\left(Y, K \mathrm{D}_{X}^{V} K(Z)\right) \\
& =g\left(Y, \mathrm{D}_{K X}^{V} K(Z)-K\left(\mathrm{D}_{X}^{V} K(Z)\right)\right.
\end{aligned}
$$

So, we get the first of the conditions (4.40). To see that this is actually equivalent to

$\mathcal{N}_{\bar{K}}^{V}(X, Y):=\mathrm{D}_{\bar{K} X}^{V} \bar{K}(Y)-\left(\bar{K} \mathrm{D}_{X}^{V} \bar{K}\right)(Y)-\mathrm{D}_{\bar{K} Y}^{V} \bar{K}(X)+\left(\bar{K} \mathrm{D}_{Y}^{V} \bar{K}\right)(X)=0$,

one has to lower an index by $g$ again, use (4.9) to arrive at an expression like $g\left(Z, \mathrm{D}_{\bar{K}}^{V} X(Y)\right)-g\left(X, \mathrm{D}_{\bar{K}} Z \frac{V}{K}(Y)\right)$. Then we take the derivatives outside $g$ to enable switching from $K$ to $\bar{K}$. We find

$$
\begin{aligned}
& g\left(Y, \mathrm{D} \frac{V}{K} X(Z)\right)-g(X, \mathrm{D} \bar{K} Y K(Z)) \\
& =g\left(\mathrm{D} \frac{V}{K} X \bar{K}(Y), Z\right)-g\left(\mathrm{D}_{\bar{K} Y}^{V} \bar{K}(X), Z\right)-\mathrm{D}_{Y}^{V} g(\bar{K} X, K Z)+\mathrm{D}_{X}^{V} g(\bar{K} Y, K Z) \\
& =g\left(\mathrm{D} \bar{K}{ }_{X}^{V} \bar{K}(Y), Z\right)-g\left(\mathrm{D}_{\bar{K} Y}^{V} \bar{K}(X), Z\right)+g\left(\mathrm{D}_{Y}^{V} \bar{K}(X), K Z\right)-g\left(\mathrm{D}_{X}^{V} \bar{K}(Y), K Z\right)
\end{aligned}
$$

where we repeatedly made use of the vertical Helmholtz condition and property (4.9).

The computation of $\omega_{\ell}\left(N_{R}\left(X^{H}, Y^{V}\right), Z^{H}\right)$ runs in a very similar way. Elimination of derivatives of $g$ requires making use of the horizontal Helmholtz condition (3.19) this time and of the property (4.10). The condition (4.41) then quite easily follows.

Consider finally $\omega_{\ell}\left(N_{R}\left(X^{H}, Y^{H}\right), Z^{H}\right)$. The first cyclic sum in (4.33) becomes

$$
\sum_{X, Y, Z} \mathrm{D}_{X}^{H}(g(U Z, K Y)-g(U Y, K Z)) .
$$

The terms involving derivatives of $g$ can be written in the form

$$
\begin{aligned}
\sum_{X, Y, Z} & \left(\mathrm{D}_{Z}^{H} g(U Y, K X)-\mathrm{D}_{X}^{H} g(U Y, K Z)\right) \\
= & \sum_{X, Y, Z}\left(\mathrm{D}_{U Y}^{H} g(Z, K X)-\mathrm{D}_{U Y}^{H} g(X, K Z)\right),
\end{aligned}
$$

in view of the Helmholtz property, after which they can be replaced by algebraic terms through (4.10) (or better its consequence (4.13)). It is then 
easy to see that, together with the remaining terms of the first cyclic sum, they make up the first two lines in the expression for (4.42). The right-hand side in this expression directly comes from what remains to be considered in the second cyclic sum of (4.33), using the bracket relation $\left[X^{H}, Y^{H}\right]=$ $[X, Y]_{H}{ }^{H}+\mathcal{R}(X, Y)^{V}$.

Equations (4.40) and (4.41) have the following meaning in terms of components with respect to the basis $\left\{H_{i}, V_{i}\right\}$ :

$$
\begin{aligned}
0= & \bar{K}_{t}^{s} V_{s}\left(K_{k}^{j}\right)-K_{s}^{j} V_{t}\left(K_{k}^{s}\right), \\
0= & K_{k}^{l} H_{l}\left(K_{j}^{i}\right)-K_{j}^{l} H_{l}\left(K_{k}^{i}\right)+K_{l}^{i}\left(H_{j}\left(K_{k}^{l}\right)-H_{k}\left(K_{j}^{l}\right)\right) \\
& +U_{k}^{l} V_{l}\left(K_{j}^{i}\right)-U_{j}^{l} V_{l}\left(K_{k}^{i}\right) .
\end{aligned}
$$

A corresponding version of the third condition, derived directly from Equation (4.42) with the aid of (4.13), can be written

$$
\sum_{i, j, k}\left(H_{k}\left(U_{i}^{l} K_{l j}-U_{j}^{l} K_{l i}\right)-\mathcal{R}_{i j}^{l} K_{l m} K_{k}^{m}\right)=0 .
$$

Finally we remark that one can manipulate (4.42) further to eliminate $g$ from it as well (i.e. to raise an index, so to speak). One will need the property (4.11) in this process; but this is a quite tedious exercise and results in an expression which is not very transparent.

\subsection{Illustrative examples}

In order to illustrate the practical applicability of the various conditions we identified, we choose to show how one can make constructive use of them in constructing recursion-type tensors related to some simple dynamics. Naturally, the simple classical system par excellence for testing new developments is the harmonic oscillator. So consider first the Lagrangian

$$
\ell=\frac{1}{2}\left(u_{1}^{2}+u_{2}^{2}\right)-\frac{1}{2}\left(q_{1}^{2}+q_{2}^{2}\right) .
$$

The metric is the Euclidian one and $\Phi=-1$, so that any choice for $K$ will commute with it. Most of the relevant conditions we have met are conditions on $K$ rather than on $J$, but it is the property (4.20) which will ensure that $K$ comes from some $J$. We wish to construct some invariant R-tensors here which will give rise to alternative Lagrangians. 
Let us first make $K$ symmetric by choosing simply $K_{12}=0$. Then (4.20) further requires that $K_{11}$ is independent of $u_{2}$ and $K_{22}$ independent of $u_{1}$, and imposing $\nabla K=0$ requires that they must be first integrals. We can take, for example

$$
K_{11}=u_{1}^{2}+q_{1}^{2}, \quad K_{22}=u_{2}^{2}+q_{2}^{2} .
$$

According to proposition 25, $K^{H}$ will be an invariant tensor and will give rise to an alternative Lagrangian, which is easily found to be

$$
\ell^{\prime}=\frac{1}{12}\left(u_{1}^{4}+u_{2}^{4}\right)+\frac{1}{2}\left(q_{1}^{2} u_{1}^{2}+q_{2}^{2} u_{2}^{2}\right)-\frac{1}{4}\left(q_{1}^{4}+q_{2}^{4}\right) .
$$

This is perhaps nothing very surprising, but observe that even for such a quite trivial example, we need a theory in which the tensor $J$ as well as $K$ are tensor fields along $\tau$. A tensor $J$ which gives rise to the above $K$ in the sense of (4.19) is given by, for example, $J_{i}^{i}=\left(q_{i}^{2}+\frac{1}{3} u_{i}^{2}\right)\left(J_{j}^{i}=0\right.$ for $i \neq j$ ), and the corresponding $U$ as defined by (4.6) is easily seen to be zero. Moreover, $N_{K}^{V}=0$, so that $K^{H}$ has vanishing Nijenhuis torsion as well.

Another symmetric $K$, which has all the properties of the preceding one, is given by

$$
K_{11}=K_{22}=u_{1} u_{2}+q_{1} q_{2}, \quad K_{12}=K_{21}=\frac{1}{2}\left(u_{1}^{2}+u_{2}^{2}+q_{1}^{2}+q_{2}^{2}\right) .
$$

So again, $R=K^{H}$ satisfies $\mathcal{L}_{\Gamma} R=0$ and $N_{R}=0$, and the corresponding Lagrangian is found to be

$$
\ell^{\prime}=\frac{1}{2} u_{1} u_{2}\left(\frac{1}{3}\left(u_{1}^{2}+u_{2}^{2}\right)+q_{1}^{2}+q_{2}^{2}\right)+\frac{1}{2} q_{1} q_{2}\left(u_{1}^{2}+u_{2}^{2}-q_{1}^{2}-q_{2}^{2}\right) .
$$

For a different example, we start from the Lagrangian $\ell=\frac{1}{2}\left(q_{1}^{2} u_{1}^{2}+u_{2}^{2}\right)$, which means that

$$
\Gamma=u_{1} \frac{\partial}{\partial q_{1}}+u_{2} \frac{\partial}{\partial q_{2}}-\frac{u_{1}^{2}}{q_{1}} \frac{\partial}{\partial u_{1}} .
$$

The only non-zero connection coefficient is $\Gamma_{1}^{1}=u_{1} / q_{1}$ (and $\Phi=0$ so that no restrictions can come from the commutation requirement in some of the propositions). The corresponding metric is

$$
g=q_{1}^{2} d q_{1} \otimes d q_{1}+d q_{2} \otimes d q_{2} .
$$

Suppose that this time our priority is to construct a tensor $R$ with vanishing torsion. Then, it may be advantageous to work with the conditions of theorem 16 on the cotangent bundle (which can be regarded also as conditions 
on $T Q$, but expressed in the variables $(q, p))$, but we will further assume from the outset that $K$ is symmetric. Recall the rather remarkable fact that for symmetric $K, \mathcal{N}_{K}^{V}=0$ (which is the same as $A_{k}^{i j}=0$ in the variables $(q, p)$ and involves 2 requirements in dimension 2$)$ is actually equivalent to the, in principle, stronger condition $N_{K}^{V}=0$ (which consists of 6 requirements in dimension 2). From the symmetry of $K$, it follows that we must have $K_{1}^{2}=q_{1}^{2} K_{2}^{1}$. Then (4.18), which expresses that $K$ comes from some $J$, implies the existence of some function $F$ such that

$$
J_{1}^{s} p_{s}=\frac{\partial F}{\partial p_{1}} \quad \text { and } \quad q_{1}^{2} J_{2}^{s} p_{s}=\frac{\partial F}{\partial p_{2}}
$$

and it follows that we will have

$$
K_{1}^{1}=\frac{\partial^{2} F}{\partial p_{1}^{2}}, \quad K_{2}^{1}=q_{1}^{-2} \frac{\partial^{2} F}{\partial p_{1} \partial p_{2}}, \quad K_{1}^{2}=\frac{\partial^{2} F}{\partial p_{1} \partial p_{2}}, \quad K_{2}^{2}=q_{1}^{-2} \frac{\partial^{2} F}{\partial p_{2}^{2}} .
$$

Using this information it is easy to see that the two independent conditions $A_{1}^{12}=A_{2}^{12}=0$ express that the ratio $\left(K_{1}^{1}-K_{2}^{2}\right) / K_{1}^{2}$ must be independent of the $p_{i}$, provided $K_{1}^{2}$ is not zero. The case $K_{1}^{2}=0$ is not very interesting and will be omitted. For $K_{1}^{2} \neq 0$, we can put

$$
K_{2}^{1}=q_{1}^{-2} K_{1}^{2}, \quad K_{2}^{2}=K_{1}^{1}-f(q) K_{1}^{2},
$$

where the last relation is actually a second-order partial differential equation for $F$. Imposing $\nabla K=0$ it immediately follows that $K_{1}^{1}$ must be a first integral $F_{1}$, that $K_{1}^{2}=q_{1} F_{2}$, where $F_{2}$ also is an as yet undetermined first integral and that $K_{2}^{2}$ is also a first integral $F_{3}$. From the last relation in (4.44) follows then that $f(q)$ must be $q_{1}^{-1}$ and $F_{3}=F_{1}-F_{2}$. In an attempt to circumvent the difficult issue of solving the equation for $F$, observe that $K_{1}^{2}=q_{1} F_{2}$ implies that $\partial F / \partial p_{2}=q_{1} \int F_{2} d p_{1}$, wherein we omit additive functions depending on only one of the $p_{i}$ because these will lead to terms in the solution which can be generated in the case $K_{1}^{2}=0$. If we use this in the expression for $K_{2}^{2}$ in terms of $F$, introduce the auxiliary function

$$
\xi=\int \frac{\partial F_{2}}{\partial p_{2}} d p_{1}
$$

and now re-express that $K_{2}^{2}$ must be a first integral, it follows that $\xi$ must solve the linear first-order equation

$$
q_{1} p_{1} \frac{\partial \xi}{\partial q_{1}}+q_{1}^{3} p_{2} \frac{\partial \xi}{\partial q_{2}}+p_{1}^{2} \frac{\partial \xi}{\partial p_{1}}=p_{1} \xi
$$


Using the method of characteristics, the general solution of this equation is found to be

$$
\xi=p_{1} \eta\left(x_{1}, x_{2}, x_{3}\right), \quad \text { with } \quad x_{1}=p_{1} / q_{1}, x_{2}=p_{2}, x_{3}=q_{2}-\frac{1}{2}\left(p_{2} / p_{1}\right) q_{1}^{3},
$$

where $\eta$ is an arbitrary function of the indicated arguments and these $x_{i}$ all are first integrals. It follows that $K_{2}^{2}=q_{1}^{-1} \xi=x_{1} \eta$. Since $F_{2}$ must itself be a first integral (and is not allowed to depend on time) it must actually be a function of the $x_{i}$ as well, and the definition of $\xi$ implies that

$$
\frac{\partial F_{2}}{\partial p_{2}}=\frac{\partial \xi}{\partial p_{1}}=\eta+x_{1} \eta_{x_{1}}+\frac{1}{2}\left(x_{2} / x_{1}\right) \eta_{x_{3}} q_{1}^{2} .
$$

Acting with $\Gamma$ on both sides, and intertwining $\Gamma$ with $\partial / \partial p_{2}$ in the left-hand side, it follows that $\left(F_{2}\right)_{x_{3}}=-x_{2} \eta_{x_{3}}$, and thus $F_{2}=-x_{2} \eta+\zeta\left(x_{1}, x_{2}\right)$ for some arbitrary $\zeta$. Returning with this information to the preceding equation, we get the restriction

$$
\zeta_{x_{2}}=2 \eta+x_{1} \eta_{x_{1}}+x_{2} \eta_{x_{2}} .
$$

Taking a derivative with respect to $x_{3}$, we get a first-order partial differential equation for $\eta_{x_{3}}$ which is easy to solve; after integration with respect to $x_{3}$ one learns that $\eta$ must be of the form

$$
\eta=x_{2}^{-2} \phi\left(x_{2} x_{1}^{-1}, x_{3}\right),
$$

for some as yet arbitrary $\phi$. In fact there is an extra freedom for adding a function of $x_{1}$ and $x_{2}$, but that can be absorbed into $\zeta$. Moreover, the preceding equation now implies that $\zeta$ cannot depend on $x_{2}$ and so we omit it (as an additive function of only one of the $p_{i}$ ). We have now come to a stage where we know that $F_{2}=-x_{2} \eta$ and

$$
K_{2}^{2}=x_{1} \eta, \quad K_{1}^{2}=q_{1} F_{2}, \quad K_{2}^{1}=q_{1}^{-1} F_{2}, \quad K_{1}^{1}=\left(x_{1}-x_{2}\right) \eta,
$$

with $\eta$ as described above. To find further specifications about $\eta$ we reimpose now that $K$ must satisfy

$$
\frac{\partial K_{2}^{1}}{\partial p_{2}}=\frac{\partial K_{2}^{2}}{\partial p_{1}}, \quad \frac{\partial K_{1}^{1}}{\partial p_{2}}=\frac{\partial K_{1}^{2}}{\partial p_{1}} .
$$

The first condition appears to be satisfied automatically, but the second gives an equation for $\phi$, with coefficients which can be expressed in terms of $x_{1}$ and $x_{2}$, except for a factor $q_{1}^{2}$ in the coefficient of $\phi_{x_{3}}$. It then follows, 
for example from acting with $\Gamma$ on the equation, that $\phi$ cannot depend on $x_{3}$, in other words must be a function of $x:=x_{2} / x_{1}$ only, and the condition reduces to

$$
\left(x-x^{2}-x^{3}\right) \phi^{\prime}=(2-x) \phi .
$$

The solution of this equation is $\phi(x)=x^{2}\left(x^{2}+x-1\right)^{-1}$. We thus have found the following type $(1,1)$ tensor

$$
K_{1}^{1}=\left(x_{1}-x_{2}\right) y^{-1}, \quad K_{2}^{2}=x_{1} y^{-1}, \quad K_{2}^{1}=-q_{1}^{-1} x_{2} y^{-1}, \quad K_{1}^{2}=-q_{1} x_{2} y^{-1},
$$

where we have put $y=x_{2}^{2}+x_{1} x_{2}-x_{1}^{2}$ for shorthand; rather surprisingly, $K$ is homogeneous of degree -1 in the $p_{i}$. This $K$ by construction satisfies all requirements for having that $R=K^{H}$ is $\Gamma$-invariant and has vanishing Nijenhuis torsion again. It is the Hessian of a Lagrangian which will be homogeneous of degree 1 and non-degenerate, but we don't have an explicit expression for this Lagrangian. Observe finally that one can easily check that also $d^{V} K=0$. This means that we are actually in the situation of proposition 27, so that the system is separable. This is not so surprising, of course, since the given system is given as decoupled equations. But in fact, the conclusion we reach here is not so trivial: it means that the given system will also separate in entirely different coordinates, namely coordinates in which $K$ diagonalises and which are guaranteed to exist by the theory in [38]. But we will not pursue this issue further.

\subsection{The case of interest when $\mathcal{L}_{\Gamma} R$ does not vanish}

We end this chapter by looking at the situation which will potentially lead to the generalisation of the concept 'special conformal Killing tensor'. It will be the basis for some further discussions on Finsler metrics in the next chapter.

As a generalisation of condition (2.10) assume that $d d_{R} E_{\ell}=\alpha \wedge d E_{\ell}$ where $\alpha$, for the time being, is an arbitrary 1-form on $T Q$. Under these circumstances, we have (cf. (4.29))

$$
i_{\mathcal{L}_{\Gamma} R} \omega=-2 d d_{R} E_{\ell}=\alpha \wedge i_{\Gamma} \omega+i_{\xi_{\alpha}} \omega \wedge d E_{\ell}
$$

where $\xi_{\alpha}$ is defined by $i_{\xi_{\alpha}} \omega=-\alpha$. The reason for splitting up the right hand side is for obtaining a tensor which is symmetric with respect to $\omega$. Observe that in all generality

$$
\begin{aligned}
i_{\alpha \otimes Z \omega}(X, Y) & =\alpha(X) \omega(Z, Y)-\alpha(Y) \omega(Z, X) \\
& =\left(\alpha \wedge i_{Z} \omega\right)(X, Y) .
\end{aligned}
$$


So the above expression can be rewritten in the form

$$
i_{\mathcal{L}_{\Gamma} R} \omega=i_{\alpha \otimes \Gamma-d E_{\ell} \otimes \xi_{\alpha}} \omega
$$

which implies

$$
\mathcal{L}_{\Gamma} R=\alpha \otimes \Gamma-d E_{\ell} \otimes \xi_{\alpha} .
$$

Indeed, it is easy to see that the right hand side of (4.45) is symmetric with respect to $\omega$ and so is $\mathcal{L}_{\Gamma} R$ since $R$ is symmetric and $\mathcal{L}_{\Gamma} \omega=0$.

In section 5.3 we will investigate relation (4.45) in the case of Lagrangian equations coming from the energy function of a Finsler metric. 


\section{Chapter 5}

\section{Application: $R$-tensors related to a Finsler metric}

In this chapter we apply the results of the previous chapter to the case where the Lagrangian system under consideration is the canonical spray of a Finsler metric. We will see that the characterisation of the R-tensor and also the requirements for an invariant $R$ and vanishing Nijenhuis tensor will simplify in this case. Moreover, as already said before, the further study of the class or R-tensors discussed in section 4.5 will lead to a generalised notion of 'special conformal Killing tensor'.

First we will recall some definitions and properties about Finsler geometry.

\subsection{Finsler spaces}

Finsler geometry is a metric generalisation of Riemannian geometry. It was in fact Riemann himself who introduced in 1854 the 'Finsler' metric structure

$$
g_{i j}=\frac{1}{2} \frac{\partial^{2} F^{2}}{\partial u^{i} \partial u^{j}}
$$

where $F$ is a function homogeneous of degree 1 in the velocities, called a Finsler function. But he restricted his research to the special case that $F(q, u)=\sqrt{a_{i j}(q) u^{i} u^{j}}$ which is known as Riemannian geometry. The study of the more general case revived in 1918 with the thesis of Finsler. In this section we will describe some basic concepts and properties, starting with the definition of a Finsler manifold.

Let $Q$ be a smooth manifold. $\mathcal{T} Q$ denotes the tangent bundle of $Q$ with the zero section deleted.

Definition 23. The pair $(Q, E)$ is said to be a Finsler manifold with energy function $E: T Q \rightarrow \mathbb{R}$ if the following conditions are satisfied 
(a) For any vector $v \in \mathcal{T} Q: E(v)>0$ and $E(0)=0$.

(b) $E$ is of class $\mathcal{C}^{1}$ on $T Q$ and smooth on $\mathcal{T} Q$.

(c) $\Delta E=\mathrm{D}_{\mathbf{T}}^{V} E=2 E$, i.e. $E$ is homogeneous of degree 2.

(d) The 2-form $\omega_{E}=d \theta_{E}=d d_{S} E$ on $\mathcal{T} Q$ is non-degenerate.

The Finsler function $F$ we just mentioned is given by $F=\sqrt{2 E}$. The metric tensor field $g$ along $\tau$, defined as Hessian of $E\left(g=\mathrm{D}^{V} \mathrm{D}^{V} E\right)$ is then homogeneous of degree 0 and satisfies the Helmholtz condition

$$
\mathrm{D}_{X}^{V} g(Y, Z)=\mathrm{D}_{Y}^{V} g(X, Z)
$$

Take $\Gamma$ to be the Euler-Lagrange field associated to $E$, called the canonical or geodesic spray of the Finsler manifold. Explicitly, we have $\Gamma=u^{k} \frac{\partial}{\partial q^{k}}-$ $2 \Gamma^{k} \frac{\partial}{\partial u^{k}}$ where

$$
\Gamma^{k}=\frac{1}{2} g^{i k}\left(u^{l} \frac{\partial^{2} E}{\partial q^{l} \partial u^{i}}-\frac{\partial E}{\partial q^{i}}\right)=\frac{1}{4} g^{i k} u^{l} u^{m}\left(\frac{\partial g_{m i}}{\partial q^{l}}+\frac{\partial g_{l i}}{\partial q^{m}}-\frac{\partial g_{l m}}{\partial q^{i}}\right) .
$$

The connection coefficients $\Gamma_{j}^{i}=\partial \Gamma^{i} / \partial u^{j}$ of the associated horizontal distribution are homogeneous of degree 1 . Remark also that the derivations $\mathrm{D}^{V}$, $\mathrm{D}^{H}$ correspond in this case to one of the four important Finsler connections, namely the Berwald connection.

Recall the following properties which are always true: $\mathrm{D}_{X}^{V} \mathbf{T}=X, \mathrm{D}_{X}^{V} \theta_{E}=$ $X\lrcorner g$ and $\nabla g=0$ (another Helmholtz condition). As a consequence of the homogeneity of $E$, we have also that

$$
\nabla \mathbf{T}=0, \quad \mathrm{D}_{X}^{H} \mathbf{T}=0,
$$

from which follows that $\nabla=\mathrm{D}_{\mathbf{T}}^{H}$ (see (3.16)). Other important consequences of the homogeneity of $E$ are listed below.

Lemma 7. When the Lagrangian $E$ is homogeneous of degree 2, we have ( $X$ and $Y$ being arbitrary vector fields along $\tau$ )

$$
\begin{gathered}
\Gamma(E)=0, \quad d^{H} E=0, \\
\mathrm{D}_{\mathbf{T}}^{V} g=0, \quad \mathrm{D}_{X}^{V} g(\mathbf{T}, Y)=0, \quad \mathrm{D}_{X}^{H} g(\mathbf{T}, Y)=0, \\
\left.\theta_{E}=\mathbf{T}\right\lrcorner g, \quad \nabla \theta_{E}=0, \quad \mathrm{D}_{X}^{H} \theta_{E}=0 .
\end{gathered}
$$


Proof. $\Gamma$ is the Euler-Lagrange field of $E$, so

$$
\Gamma\left(\frac{\partial E}{\partial u^{i}}\right)-\frac{\partial E}{\partial q^{i}}=0 .
$$

It follows from the homogeneity that $\Gamma(E)=0$ and subsequently, interchanging the partial derivatives in the first term $\left(\left[\Gamma, \frac{\partial}{\partial u^{i}}\right]=-H_{i}+\Gamma_{i}^{k} \frac{\partial}{\partial u^{k}}\right)$, the condition can be rewritten as $H_{i}(E)=0$ or $d^{H} E=0$.

$g=\mathrm{D}^{V} \mathrm{D}^{V} E$, so $g$ is homogeneous of degree 0 , i.e $\mathrm{D}_{\mathbf{T}}^{V} g=0$. It then follows from (5.1) that $\mathrm{D}_{X}^{V} g(\mathbf{T}, Y)=0$. By taking the dynamical covariant derivative $\nabla$ of this last equation, we get

$$
0=\nabla \mathrm{D}_{X}^{V} g(\mathbf{T}, Y)=\mathrm{D}_{X}^{H} g(\mathbf{T}, Y)
$$

because $\nabla g=0, \nabla \mathbf{T}=0$ and $\left[\nabla, \mathrm{D}_{X}^{V}\right]=\mathrm{D}_{\nabla X}^{V}-\mathrm{D}_{X}^{H}$.

The homogeneity of $E$ implies also that

$$
\theta_{E}=\frac{\partial E}{\partial u^{i}} d q^{i}=\frac{\partial^{2} E}{\partial u^{i} \partial u^{k}} u^{k} d q^{i}
$$

or $\left.\theta_{E}=\mathbf{T}\right\lrcorner g$. In view of $\nabla \mathbf{T}=0$ and $\nabla g=0$, it then immediately follows that $\nabla \theta_{E}=0$ and as a consequence of $\mathrm{D}_{X}^{H} g(\mathbf{T}, Y)=0$ and $\mathrm{D}_{X}^{H} \mathbf{T}=0$, we find that $\mathrm{D}_{X}^{H} \theta_{E}=0$.

For later use we come back in more detail to the relevance of the second relation of (5.3) and recall the following properties.

Lemma 8. $d^{H} E=0$ is necessary and sufficient for $\Gamma$ to be the EulerLagrange field of $E$. Moreover, with $g=\mathrm{D}^{V} \mathrm{D}^{V} E$, this is further equivalent to $\nabla g=0$.

Proof. That $d^{H} E=0$ is a necessary condition is proven in lemma 7 . For the converse: $d^{H} E=0$ implies $\Gamma(E)=0$ since $\nabla=\mathrm{D}_{\mathrm{T}}^{H}$. With the same argumentation as in lemma 7, we see that $H_{i}(E)=0$ is the same as (5.6).

For the second part of the statement: if $\Gamma$ is the Euler-Lagrange field of $E$, then $g=\mathrm{D}^{V} \mathrm{D}^{V} E$ obviously satisfies the Helmholtz conditions, hence in particular $\nabla g=0$. Conversely, with $g=\mathrm{D}^{V} \mathrm{D}^{V} E$, we have from homogeneity of $E$ that

$$
E=\frac{1}{2} g(\mathbf{T}, \mathbf{T}) .
$$

Then, $\nabla g=0$ implies in the first place that $0=\nabla g(\mathbf{T}, \mathbf{T})=\nabla(g(\mathbf{T}, \mathbf{T}))=$ $2 \Gamma(E)=0$, and secondly

$0=\nabla g(\mathbf{T}, X)=\nabla \mathrm{D}_{X}^{V} E-\mathrm{D}_{\nabla X}^{V} E=\left(\mathrm{D}_{X}^{V} \nabla-\mathrm{D}_{X}^{H}\right)(E)=-\mathrm{D}_{X}^{H} E=-d^{H} E(X)$, 
where we have used the fact that $g(\mathbf{T}, X)=\theta_{E}(X)=\mathrm{D}_{X}^{V} E$.

Remark that the third Helmholtz condition

$$
g(\Phi(X), Y)=g(X, \Phi(Y))
$$

is in this case redundant. That this condition is automatically satisfied can also be proven as follows. From

$$
0=\left[\mathrm{D}_{X}^{H}, \mathrm{D}_{Y}^{H}\right] E=\mathrm{D}_{\mathcal{R}(X, Y)}^{V} E=g(\mathbf{T}, \mathcal{R}(X, Y)),
$$

we have that

$$
g(\mathbf{T}, \mathcal{R}(\mathbf{T}, X))=g(\mathbf{T}, \Phi(X))=0 .
$$

Taking the derivative $\mathrm{D}_{Y}^{V}$ of this equation, we find that

$$
g(Y, \Phi(X))+g\left(\mathbf{T},\left(D_{Y}^{V} \Phi\right) X\right)=0
$$

and because $d^{V} \Phi=3 \mathcal{R}$, it follows that

$$
g(Y, \Phi(X))-g(X, \Phi(Y))+3 g(\mathbf{T}, \mathcal{R}(Y, X))=0
$$

or

$$
g(\Phi(X), Y)=g(X, \Phi(Y)) .
$$

\subsection{R-tensors in the Finsler case}

We are now in a position to specialise the interesting results of chapter 4 to the case of a Finslerian metric, which is the same as generalising the results of chapter 3 from the Riemannian to the Finsler case. So, without changing notations, it will from now on be understood that the tangent bundle $T Q$ has its zero section removed and that we have a Finsler metric $g$. We will assume that the type $(1,1)$ tensor field $J$ along $\tau$ we start from, is homogeneous of degree zero. We then immediately recover the Riemannian situation when 'homogeneous of degree zero' is specialised to 'independent of the velocities' both for the metric $g$ and the tensor $J$.

First we want to analyse the effect of assuming that the $J$ we start from is homogeneous of degree zero, i.e. $\mathrm{D}_{\mathbf{T}}^{V} J=0$, on the tensor $R$ defined by (4.4). The definition of the tensor fields $K$ and $U$ can be found in proposition 20 . 
Proposition 29. If $g$ and $J$ are homogeneous of degree 0 , then $K$ is homogeneous of degree 0 and $U$ is homogeneous of degree 1. Moreover, we have $K \theta_{E}=J \theta_{E}$ and the defining relation of $U$ simplifies to

$$
g(U X, Y)=g\left(\mathbf{T}, d^{H} J(X, Y)\right)=g\left(\mathbf{T}, d^{H} K(X, Y)\right) .
$$

Proof. The homogeneity properties of $K$ and $U$ follow from acting with $\mathrm{D}_{\mathbf{T}}^{V}$ their defining relation. Taking $Y=\mathbf{T}$ in the defining relation of $K$, we immediately have that $g(K X, \mathbf{T})=g(J X, \mathbf{T})$ or $K \theta_{E}=J \theta_{E}$. Finally, the simplification in the defining relation for $U$ immediately follows from the fact that $\mathrm{D}_{X}^{H} \theta_{E}=0$, so that $d^{H}\left(J \theta_{E}\right)(X, Y)=\theta_{E}\left(d^{H} J(X, Y)\right)$.

This proposition is quite important. We have seen in subsection 4.2.1 that if we have a type $(1,1)$ tensor field $K$ which satisfies (4.9), i.e. $K=\mathrm{D}^{V} \beta$ with $\beta$ a 1-form, then $\beta=J \theta_{E}$ is not totally determined. So, the corresponding tensor field $U$ is not fixed either. Even if we assume that $K$ has to be homogeneous of degree 0 , we can still add an arbitrary basic 1 -form $\beta_{0}$ to $\beta$. But in proposition 29 , we consider the situation that $K$ comes from a type $(1,1)$ tensor field $J$ homogeneous of degree 0 . In other words, $\beta$ has to be homogeneous of degree 1 . In that case, the 1 -form $\beta=J \theta_{E}=K \theta_{E}$ is totally determined and the tensor field $U$ is given by (5.8).

Further we remark that because of the homogeneity of $K$ it follows from (4.9) that

$$
g\left(\mathrm{D}_{Y}^{V} K(X), \mathbf{T}\right)=0
$$

and the commutator property $\left[d^{V}, \nabla\right]=d^{H}$ leads then to the following expression for $U$ :

$$
g(U X, Y)=g\left(\mathbf{T}, d^{V} \nabla K(X, Y)\right) .
$$

We now come back to the two aspects of recursion we have studied in chapter 4 and investigate what the homogeneity properties of the Finsler case can do to simplify the conditions for vanishing $\mathcal{L}_{\Gamma} R$ or $N_{R}$.

Theorem 18. Assume that $g$ and $J$ are homogeneous of degree 0 . Then, if $K$ is symmetric and $\nabla K=0$, we have automatically that $U=0$ and $\Phi K=K \Phi$. In other words, the necessary and sufficient conditions for having $\mathcal{L}_{\Gamma} R=0$ (see theorem 14 ) reduce to $K=\bar{K}$ and $\nabla K=0$.

Proof. We know from proposition 32 and the homogeneity that $K=\bar{K}$ implies $d^{V}\left(J \theta_{E}\right)=d^{V}\left(K \theta_{E}\right)=0$. Since $\left[\nabla, d^{V}\right]=-d^{H}$, it then follows from 
the assumption $\nabla K=0$ and the property $\nabla \theta_{E}=0$ that also $d^{H}\left(K \theta_{E}\right)=$ $d^{H}\left(J \theta_{E}\right)=0$, whence $U=0$.

Showing that $\Phi$ will commute with $K$ goes similar as in the proof of proposition 17 . The property (4.19), which roughly expresses that $K$ comes from a $J$, plus (4.20), ensure for a symmetric $K$ that $K_{i j}$ is a Hessian of some function, and we can actually determine such a function in the Finsler case. Indeed, from the symmetry of $K$ and the homogeneity, we have that

$$
\frac{\partial\left(J_{j}^{l} p_{l} u^{j}\right)}{\partial u^{k}}=J_{k}^{l} p_{l}+u^{j} \frac{\partial J_{j}^{l} p_{l}}{\partial u^{k}}=J_{k}^{l} p_{l}+u^{j} \frac{\partial J_{k}^{l} p_{l}}{\partial u^{j}}=2 J_{k}^{l} p_{l},
$$

so that $K_{i j}$ is the Hessian of the function

$$
\mathcal{K}:=\frac{1}{2} J_{j}^{l} p_{l} u^{j}=\frac{1}{2} K_{j}^{l} p_{l} u^{j} \quad \text { or in intrinsic terms } \quad \mathcal{K}=\frac{1}{2}\left(K \theta_{E}\right)(\mathbf{T}) .
$$

Further we have that $\mathcal{K}$ is a first integral as a consequence of $\nabla \mathbf{T}=0$, $\nabla \theta_{E}=0$ and $\nabla K=0$. Moreover, the above computation expresses that $d^{V} \mathcal{K}=K \theta_{E}$ and thus

$$
0=\nabla d^{V} \mathcal{K}=d^{V} \nabla \mathcal{K}-d^{H} \mathcal{K}=-d^{H} \mathcal{K} .
$$

But in the case of a spray, $d^{H} \mathcal{K}=0$ is necessary and sufficient for $\mathcal{K}$ to be a Lagrangian for the system (see (3.21)). Hence $\Phi$ is symmetric with respect to its Hessian $K$. Since $\Phi$ and $K$ are symmetric with respect to $g$, it follows from $K_{m i} \Phi_{j}^{m}=K_{m j} \Phi_{i}^{m}$ that

$$
g_{i s} K_{m}^{s} \Phi_{j}^{m}=g_{m s} K_{i}^{s} \Phi_{j}^{m}=g_{m s} K_{j}^{s} \Phi_{i}^{m}=g_{m i} K_{j}^{s} \Phi_{s}^{m} .
$$

Multiplying this with $g^{i r}$, we find that $K_{m}^{r} \Phi_{j}^{m}=K_{j}^{s} \Phi_{s}^{r}$, i.e. the type $(1,1)$ tensor $K$ commutes with $\Phi$.

The conditions for vanishing Nijenhuis torsion also simplify in the Finsler case.

Theorem 19. If $g$ and $J$ are homogeneous of degree 0 , we have $N_{R}=0$ if and only if the coefficients $A_{k}^{i j}$ and $B_{k j}^{i}$ (see (4.37) and (4.38)) vanish, or equivalently

$$
\begin{gathered}
\mathcal{N}_{\bar{K}}^{V}=0 \quad \text { and } \\
\mathcal{N}_{K}^{H}(X, Y)+\mathrm{D}_{U X}^{V} K(Y)-\mathrm{D}_{U Y}^{V} K(X)=0 .
\end{gathered}
$$


Proof. We go back to the equivalent calculation of $N_{\widetilde{J}}$ on $T^{*} Q$, knowing that by homogeneity: $J_{j}^{l} p_{l}=K_{j}^{l} p_{l}$ and $p_{i} \partial K_{j}^{i} / \partial p_{k}=0$. Multiplying condition (4.38) by $p_{i}$, we thus get:

$$
K_{k}^{l} \frac{\partial\left(K_{j}^{i} p_{i}\right)}{\partial q^{l}}-K_{j}^{l} \frac{\partial\left(K_{k}^{i} p_{i}\right)}{\partial q^{l}}+K_{l}^{i} p_{i} \frac{\partial M_{j k}}{\partial p_{l}}=0
$$

Taking a further derivative with respect to $q^{m}$, followed by a cyclic sum over the free indices, it follows that

$$
\begin{aligned}
0= & \sum_{j, k, m}\left[\frac{\partial K_{k}^{l}}{\partial q^{m}}\left(\frac{\partial\left(K_{j}^{i} p_{i}\right)}{\partial q^{l}}-\frac{\partial\left(K_{l}^{i} p_{i}\right)}{\partial q^{j}}\right)-\frac{\partial K_{j}^{l}}{\partial q^{m}}\left(\frac{\partial\left(K_{k}^{i} p_{i}\right)}{\partial q^{l}}-\frac{\partial\left(K_{l}^{i} p_{i}\right)}{\partial q^{k}}\right)\right. \\
& \left.+K_{k}^{l}\left(\frac{\partial^{2}\left(K_{j}^{i} p_{i}\right)}{\partial q^{l} \partial q^{m}}-\frac{\partial^{2}\left(K_{m}^{i} p_{i}\right)}{\partial q^{l} \partial q^{j}}\right)\right] \\
= & \sum_{j, k, m}\left[\frac{\partial K_{k}^{l}}{\partial q^{m}} M_{l j}-\frac{\partial K_{j}^{l}}{\partial q^{m}} M_{l k}+K_{k}^{l} \frac{\partial M_{m j}}{\partial q^{l}}\right] \\
= & \sum_{j, k, m}\left[K_{k}^{l} \frac{\partial M_{m j}}{\partial q^{l}}+M_{k l} \frac{\partial M_{m j}}{\partial p_{l}}\right]
\end{aligned}
$$

where we use the properties (4.36). So, the third condition in theorem 16, in the Finsler case, is automatically satisfied in view of the second.

\subsection{Generalisation of the SCK condition}

We now look at the case that $d d_{R} E=\alpha \wedge d E$ with $\alpha$ a 1-form on $T Q$. As seen in section 4.5 (where $E=E_{\ell}$ ), this implies the following relation

$$
\mathcal{L}_{\Gamma} R=\alpha \otimes \Gamma-d E \otimes \xi_{\alpha}
$$

Put $\alpha=\alpha_{1}{ }^{H}+\alpha_{2}{ }^{V}$, where $\alpha_{1}$ and $\alpha_{2}$ are two 1-forms along $\tau$; in coordinates: $\alpha=\alpha_{1 i} d q^{i}+\alpha_{2 i} \eta^{i}$. Similarly, $\xi_{\alpha}$ can be written as $A_{1}{ }^{V}+A_{2}{ }^{H}$ with $A_{1}$ and $A_{2}$ vector fields along $\tau$. Hence $A_{1}$ is defined by

$\omega\left(\xi_{\alpha}, Z^{H}\right)=\omega\left(A_{1}^{V}, Z^{H}\right)=g\left(A_{1}, Z\right)=-\alpha\left(Z^{H}\right)=-\alpha_{1}(Z)$ or $\left.A_{1}\right\lrcorner g=-\alpha_{1}$.

In coordinates this means

$$
A_{1}=-g^{i k} \alpha_{1 k} \frac{\partial}{\partial q^{i}}
$$


In the same way we find that

$$
\left.A_{2}\right\lrcorner g=\alpha_{2} \quad \text { or in coordinates } \quad A_{2}=g^{i k} \alpha_{2 k} \frac{\partial}{\partial q^{i}}
$$

Now, using the decomposition of $\mathcal{L}_{\Gamma} R$ as established in theorem 14, we arrive at the following results.

Theorem 20. If $(Q, E)$ is a Finsler manifold and $\Gamma$ is the canonical spray, then $\mathcal{L}_{\Gamma} R=\alpha \otimes \Gamma-d E \otimes \xi_{\alpha}$ for some 1-form $\alpha$ on $T Q$, if and only if the following conditions are satisfied

$$
\begin{aligned}
K-\bar{K}= & \alpha_{2} \otimes \mathbf{T}-\theta_{E} \otimes A_{2}, \\
\nabla K= & \frac{1}{2}\left[\left(\alpha_{1}+\nabla \alpha_{2}\right) \otimes \mathbf{T}-\theta_{E} \otimes\left(A_{1}+\nabla A_{2}\right)\right], \\
U= & \frac{1}{2}\left[\left(\nabla \alpha_{2}-\alpha_{1}\right) \otimes \mathbf{T}-\theta_{E} \otimes\left(A_{1}+\nabla A_{2}\right)\right], \\
{[\Phi, K]=} & -\frac{1}{2}\left[\left(\nabla^{2} \alpha_{2}-\nabla \alpha_{1}\right) \otimes \mathbf{T}-\theta_{E} \otimes\left(\nabla A_{1}+\nabla^{2} A_{2}\right)\right] \\
& -\Phi\left(\alpha_{2}\right) \otimes \mathbf{T} .
\end{aligned}
$$

Proof. This proof is similar to the proof of theorem 13. If we evaluate the right-hand side of (5.11) on a vertical lift of a vector field $X$, we get $\alpha_{2}(X) \Gamma-d^{V} E(X) \xi_{\alpha}$. Since $\Gamma=\mathbf{T}^{H}$ and $\xi_{\alpha}=A_{1}{ }^{V}+A_{2}{ }^{H}$ this results in

$$
\mathcal{L}_{\Gamma} R\left(X^{V}\right)=\left(\alpha_{2} \otimes \mathbf{T}-d^{V} E \otimes A_{2}\right)(X)^{H}-\left(d^{V} E \otimes A_{1}\right)(X)^{V} .
$$

Comparing this with (4.24), we find the following conditions:

$$
\begin{aligned}
K-\bar{K} & =\alpha_{2} \otimes \mathbf{T}-d^{V} E \otimes A_{2} \\
\nabla \bar{K}+U & =-d^{V} E \otimes A_{1}
\end{aligned}
$$

The first relation is the required condition (5.12) and we use this in (5.17) to get that

$$
\nabla K+U=\nabla \alpha_{2} \otimes \mathbf{T}-d^{V} E \otimes\left(A_{1}+\nabla A_{2}\right) .
$$

For the evaluation of (5.11) on a vector field $X^{H}$, we work in the same way and find that

$$
\mathcal{L}_{\Gamma} R\left(X^{H}\right)=\left(\alpha_{1} \otimes \mathbf{T}\right)(X)^{H}
$$

because $d^{H} E=0$ and $\Gamma=\mathbf{T}^{H}$. Comparison with (4.25) reveals the requirements

$$
\nabla K-U=\alpha_{1} \otimes \mathbf{T}
$$


and

$$
\Phi \circ K-\bar{K} \circ \Phi+\nabla U=0 .
$$

Bringing the expressions (5.19) and (5.18) together, gives us immediately relations (5.13) and (5.14). Further we have that

$$
\nabla U=\frac{1}{2}\left[\left(\nabla^{2} \alpha_{2}-\nabla \alpha_{1}\right) \otimes \mathbf{T}-d^{V} E \otimes\left(\nabla A_{1}+\nabla^{2} A_{2}\right)\right]
$$

which leads together with (5.20) and (5.16) to condition (5.15) because $\Phi(\mathbf{T})=0$.

Note that, if $K$ is homogeneous of degree 0 , it follows from (5.19) that $\alpha_{1}$ has to be homogeneous of degree 0 . From (5.13) we get then that $\nabla \alpha_{2}$ is also homogeneous of degree 0. Now, we take the trace of expression (5.13). Because $\theta_{E}\left(A_{1}\right)=-\alpha_{1}(\mathbf{T})$ and $\theta_{E}\left(\nabla A_{2}\right)=\nabla\left(\theta_{E}\left(A_{2}\right)\right)=\nabla \alpha_{2}(\mathbf{T})$, one obtains that

$$
\alpha_{1}(\mathbf{T})=\nabla \operatorname{tr} K
$$

Note further that condition (5.12) in fact says that $K$ is symmetric if and only if $\theta_{E} \otimes \alpha_{2}$ is symmetric. Since $\Phi_{s}^{j}\left(\partial E / \partial u^{j}\right)=\Phi_{s}^{j} g_{j m} u^{m}=\Phi_{m}^{j} u^{m} g_{i s}=0$, we have that if $\bar{K}=K$, the conditions (5.13), (5.14) and (5.15) become

$$
\begin{aligned}
\nabla K & =\frac{1}{2}\left[\alpha_{1} \otimes \mathbf{T}-\theta_{E} \otimes A_{1}\right] \\
U & =-\frac{1}{2}\left[\alpha_{1} \otimes \mathbf{T}+\theta_{E} \otimes A_{1}\right] \\
{[\Phi, K] } & =\frac{1}{2}\left[\nabla \alpha_{1} \otimes \mathbf{T}+\theta_{E} \otimes \nabla A_{1}\right]
\end{aligned}
$$

In coordinates the first one is given by

$$
\nabla K_{m i}=\frac{1}{2} u^{k}\left[g_{m k} \alpha_{1 i}+g_{i k} \alpha_{1 m}\right]
$$

which has the same form as the special conformal Killing tensor condition (3.68) and also reduces to it in case of a Riemannian metric and basic tensor $J$.

Remark that in the Riemannian case, the conditions (5.14) and (5.15) were satisfied automatically. This will not be the case here. But the fact that $J$ is homogeneous of degree 0 , will lead to some simplifications. The first simplification, about the condition on $U$, is quite easy to find. 
Proposition 30. Suppose that $J$ is homogeneous of degree 0 and $K$ satisfies condition (5.13). Then

$$
U=\frac{1}{2}\left[\left(\nabla \alpha_{2}-\alpha_{1}\right) \otimes \mathbf{T}-\theta_{E} \otimes\left(A_{1}+\nabla A_{2}\right)\right]
$$

if and only if

$$
\nabla \alpha_{2}(Y)=\mathrm{D}_{Y}^{V} \alpha_{1}(\mathbf{T})
$$

Proof. Since $d^{V} \mathbf{T}=1$ and $\left(d^{V}\right)^{2}=0$, we have that

$$
\begin{aligned}
& d^{V} \nabla K= \\
& \quad \frac{1}{2}\left[\left(d^{V} \alpha_{1}+d^{V} \nabla \alpha_{2}\right) \otimes \mathbf{T}-\left(\alpha_{1}+\nabla \alpha_{2}\right) \wedge 1+d^{V} E \wedge\left(d^{V} A_{1}+d^{V} \nabla A_{2}\right)\right] .
\end{aligned}
$$

Putting this in expression (5.9), we get that $U$ is given by (5.14) if and only if

$$
\begin{aligned}
0= & g(\mathbf{T}, \mathbf{T})\left(\mathrm{D}_{X}^{V} \alpha_{1}(Y)+\mathrm{D}_{X}^{V} \nabla \alpha_{2}(Y)-\mathrm{D}_{Y}^{V} \alpha_{1}(X)-\mathrm{D}_{Y}^{V} \nabla \alpha_{2}(X)\right) \\
& +g(X, \mathbf{T})\left(2 \nabla \alpha_{2}(Y)-\mathrm{D}_{Y}^{V} \alpha_{1}(\mathbf{T})+\mathrm{D}_{Y}^{V} \nabla \alpha_{2}(\mathbf{T})\right) \\
& -g(Y, \mathbf{T})\left(2 \nabla \alpha_{2}(X)-\mathrm{D}_{X}^{V} \alpha_{1}(\mathbf{T})+\mathrm{D}_{X}^{V} \nabla \alpha_{2}(\mathbf{T})\right)
\end{aligned}
$$

In this condition we set $X=\mathbf{T}$ and find

$$
g(\mathbf{T}, \mathbf{T})\left(-\mathrm{D}_{Y}^{V} \alpha_{1}(\mathbf{T})+\nabla \alpha_{2}(Y)\right)=g(Y, \mathbf{T}) \nabla \alpha_{2}(\mathbf{T})
$$

because $\alpha_{1}$ and $\nabla \alpha_{2}$ are homogeneous of degree 0 . Taking $\mathrm{D}_{X}^{V}$ of this equation we get

$$
\begin{aligned}
0= & g(\mathbf{T}, \mathbf{T})\left(\mathrm{D}_{X}^{V} \alpha_{1}(Y)+\mathrm{D}_{X}^{V} \nabla \alpha_{2}(Y)-\mathrm{D}_{Y}^{V} \alpha_{1}(X)-\mathrm{D}_{Y}^{V} \nabla \alpha_{2}(X)\right) \\
& +g(X, \mathbf{T})\left(3 \nabla \alpha_{2}(Y)-2 \mathrm{D}_{Y}^{V} \alpha_{1}(\mathbf{T})+\mathrm{D}_{Y}^{V} \nabla \alpha_{2}(\mathbf{T})\right) \\
& -g(Y, \mathbf{T})\left(3 \nabla \alpha_{2}(X)-2 \mathrm{D}_{X}^{V} \alpha_{1}(\mathbf{T})+\mathrm{D}_{X}^{V} \nabla \alpha_{2}(\mathbf{T})\right) .
\end{aligned}
$$

Comparison with (5.26) implies that (5.25) is a necessary condition. It is also easy to see that (5.25) is sufficient.

Remark that condition (5.25) implies that $\nabla \alpha_{2}(\mathbf{T})$ has to vanish. Furthermore, if we assume that $K$ is symmetric, it follows from (5.12) that $\nabla \alpha_{2}(\mathbf{T}) \mathbf{T}=2 E \nabla A_{2}$. So $\nabla \alpha_{2}(\mathbf{T})=0$ implies that $\nabla A_{2}=0$, from which follows that $\nabla \alpha_{2}=0$. Inserting this in condition (5.25), we find that $\mathrm{D}_{X}^{V} \alpha_{1}(\mathbf{T})=0$. Taking $\mathrm{D}_{Y}^{V}$ of this equation, we obtain the following relation

$$
d^{V} \alpha_{1}=0 .
$$


So, if $K$ is symmetric, condition (5.14) is equivalent to $d^{V} \alpha_{1}=0$.

The homogeneity of $J$ simplifies also the condition on the commutator of $K$ and $\Phi$. But this is a lot more complicated to proof.

Proposition 31. Suppose that $K$ is homogeneous of degree 0, satisfies (5.12) and (5.13) and $\nabla \alpha_{2}(Y)=\mathrm{D}_{Y}^{V} \alpha_{1}(\mathbf{T})$. Then

$$
[\Phi, K]=-\frac{1}{2}\left[\left(\nabla^{2} \alpha_{2}-\nabla \alpha_{1}\right) \otimes \mathbf{T}-\theta_{E} \otimes\left(\nabla A_{1}+\nabla^{2} A_{2}\right)\right]-\Phi\left(\alpha_{2}\right) \otimes \mathbf{T}
$$

if and only if

$$
\nabla \alpha_{1}(Y)=\alpha_{2}(\Phi(Y))+\mathrm{D}_{Y}^{H} \alpha_{1}(\mathbf{T})
$$

Proof. From $g\left(\mathrm{D}_{Y}^{V} K(X), \mathbf{T}\right)=0$ follows that

$$
g\left(\mathrm{D}_{Y}^{H} K(X), \mathbf{T}\right)=g\left(\mathrm{D}_{Y}^{V} \nabla K(X), \mathbf{T}\right)
$$

because $\nabla g=0, \nabla \mathbf{T}=0$ and $\left[\nabla, \mathrm{D}_{Y}^{V}\right]=\mathrm{D}_{\nabla Y}^{V}-\mathrm{D}_{Y}^{H}$. Taking the dynamical covariant derivative of this expression and using the commutator properties $\left[\nabla, \mathrm{D}_{Y}^{H}\right]=\mathrm{D}_{\nabla Y}^{H}+\mathrm{D}_{\mathcal{R}(\mathbf{T}, Y)}^{V}+\mu_{R i e(\mathbf{T}, Y)}$ and $\left[\nabla, \mathrm{D}_{Y}^{V}\right]=\mathrm{D}_{\nabla Y}^{V}-\mathrm{D}_{Y}^{H}$ we get

$$
\begin{aligned}
& g\left(\mathrm{D}_{Y}^{H} \nabla K(X)+\mathrm{D}_{\mathcal{R}(\mathbf{T}, Y)}^{V} K(X)+\mu_{R i e(\mathbf{T}, Y)} K(X), \mathbf{T}\right) \\
& \quad=g\left(\mathrm{D}_{Y}^{V} \nabla^{2} K(X)-\mathrm{D}_{Y}^{H} \nabla K(X), \mathbf{T}\right) .
\end{aligned}
$$

The second term vanishes because of (4.9), so this becomes

$$
g\left(\mu_{R i e(\mathbf{T}, Y)} K(X), \mathbf{T}\right)=g\left(\mathrm{D}_{Y}^{V} \nabla^{2} K(X)-2 \mathrm{D}_{Y}^{H} \nabla K(X), \mathbf{T}\right) .
$$

We will evaluate both sides of this equality now separately. The left hand side, using the techniques for the calculation of $\mu_{R i e(\mathbf{T}, y)} K$ similar to what we did in the proof of theorem 13, can be written in the first place as

$$
\begin{aligned}
& g\left(\mu_{\operatorname{Rie}(\mathbf{T}, Y)} K(X), \mathbf{T}\right)= \\
& \quad-g\left(\mathrm{D}_{K X}^{V} \Phi(Y)-\mathcal{R}(K X, Y)+K \mathrm{D}_{X}^{V} \Phi(Y)-K \mathcal{R}(X, Y), \mathbf{T}\right) .
\end{aligned}
$$

Taking into account that $d^{V} \Phi=3 \mathcal{R}, \Phi(\mathbf{T})=0$ and $\bar{\Phi}=\Phi$ we subsequently find

$$
\begin{aligned}
& g\left(\mu_{R i e(\mathbf{T}, Y)} K(X), \mathbf{T}\right)= \\
& \frac{1}{3}\left[3 g(\bar{K} \Phi(Y), X)-2 g(K \Phi(Y), X)-g(\Phi \bar{K}(Y), X)+2 \mathrm{D}_{X}^{V}(g(\mathbf{T}, K \Phi(Y)))\right. \\
& \left.-2 g\left(\mathbf{T}, K \Phi\left(\mathrm{D}_{X}^{V} Y\right)\right)+\mathrm{D}_{Y}^{V}(g(\mathbf{T}, K \Phi(X)))-g\left(\mathbf{T}, K \Phi\left(\mathrm{D}_{Y}^{V} X\right)\right)\right] .
\end{aligned}
$$


And since $\bar{K}=K-\alpha_{2} \otimes \mathbf{T}+d^{V} E \otimes A_{2}$, by assumption, this finally becomes

$$
\begin{aligned}
& g\left(\mu_{\operatorname{Rie}(\mathbf{T}, Y)} K(X), \mathbf{T}\right)= \\
& \quad \frac{1}{3}\left[g([K, \Phi](Y), X)-g(X, \mathbf{T}) \alpha_{2}(\Phi(Y))\right. \\
& \quad-g(Y, \mathbf{T}) \alpha_{2}(\Phi(X))+2 \mathrm{D}_{X}^{V}(g(\mathbf{T}, K \Phi(Y)))-2 g\left(\mathbf{T}, K \Phi\left(\mathrm{D}_{X}^{V} Y\right)\right) \\
& \left.\quad+\mathrm{D}_{Y}^{V}(g(\mathbf{T}, K \Phi(X)))-g\left(\mathbf{T}, K \Phi\left(\mathrm{D}_{Y}^{V} X\right)\right)\right]
\end{aligned}
$$

where we now see the commutator $[\Phi, K]$ appear.

Using (5.13), the right hand side of equation (5.29) becomes

$$
\begin{aligned}
& 2 g\left(\mathrm{D}_{Y}^{V} \nabla^{2} K(X), \mathbf{T}\right)-4 g\left(\mathrm{D}_{Y}^{H} \nabla K(X), \mathbf{T}\right)= \\
& \quad g(X, Y)\left[\nabla \alpha_{1}(\mathbf{T})-\nabla^{2} \alpha_{2}(\mathbf{T})\right]+g(Y, \mathbf{T})\left[\nabla \alpha_{1}(X)+\nabla^{2} \alpha_{2}(X)\right] \\
& \quad+g(X, \mathbf{T})\left[\mathrm{D}_{Y}^{V} \nabla \alpha_{1}(\mathbf{T})-2 \mathrm{D}_{Y}^{H} \alpha_{1}(\mathbf{T})-\mathrm{D}_{Y}^{V} \nabla^{2} \alpha_{2}(\mathbf{T})+2 \mathrm{D}_{Y}^{H} \nabla \alpha_{2}(\mathbf{T})\right] \\
& \quad+g(\mathbf{T}, \mathbf{T})\left[\mathrm{D}_{Y}^{V} \nabla \alpha_{1}(X)+\mathrm{D}_{Y}^{V} \nabla^{2} \alpha_{2}(X)-2 \mathrm{D}_{Y}^{H} \alpha_{1}(X)-2 \mathrm{D}_{Y}^{H} \nabla \alpha_{2}(X)\right] .
\end{aligned}
$$

So, the equality (5.29) at this point has been replaced by the relation which arises form equating the right hand side of (5.30) and (5.31). We next further simplify this relation as follows. First we evaluate it for $X=\mathbf{T}$, taking into account that $\Phi$ is homogeneous of degree 2. We obtain

$$
\begin{aligned}
& 2 g(\mathbf{T}, K \Phi(Y)) \\
& \quad=g(Y, \mathbf{T}) \nabla \alpha_{1}(\mathbf{T})+g(\mathbf{T}, \mathbf{T})\left(\mathrm{D}_{Y}^{V} \nabla \alpha_{1}(\mathbf{T})-2 \mathrm{D}_{Y}^{H} \alpha_{1}(\mathbf{T})+\alpha_{2}(\Phi(Y))\right) .
\end{aligned}
$$

Now use this expression to simplify a number of terms in the relation under consideration. Then it can readily be seen that the condition (5.27) about the commutator $[\phi, K]$ is equivalent to

$$
\begin{aligned}
0= & \frac{1}{2} g(X, Y) \nabla^{2} \alpha_{2}(\mathbf{T})+\frac{1}{6} g(X, \mathbf{T})\left[2 \mathrm{D}_{Y}^{V} \nabla \alpha_{1}(\mathbf{T})+3 \mathrm{D}_{Y}^{V} \nabla^{2} \alpha_{2}(\mathbf{T})\right. \\
& \left.-2 \mathrm{D}_{Y}^{H} \alpha_{1}(\mathbf{T})-6 \mathrm{D}_{Y}^{H} \nabla \alpha_{2}(\mathbf{T})+\nabla^{2} \alpha_{2}(Y)\right] \\
& +\frac{2}{3} g(Y, \mathbf{T})\left[\mathrm{D}_{X}^{V} \nabla \alpha_{2}(\mathbf{T})-\mathrm{D}_{X}^{H} \alpha_{1}(\mathbf{T})-\nabla^{2} \alpha_{2}(X)\right] \\
& +\frac{1}{6} g(\mathbf{T}, \mathbf{T})\left[3\left(\mathrm{D}_{X}^{V} \mathrm{D}_{Y}^{V}-\mathrm{D}_{\mathrm{D}_{X}^{V} Y}^{V}\right) \nabla \alpha_{1}(\mathbf{T})-\mathrm{D}_{Y}^{V} \nabla \alpha_{1}(X)\right. \\
& -4\left(\mathrm{D}_{X}^{V} \mathrm{D}_{Y}^{H}-\mathrm{D}_{\mathrm{D}_{X}^{H} Y}^{H}\right) \alpha_{1}(\mathbf{T})+2 \mathrm{D}_{Y}^{H} \alpha_{1}(X)+\mathrm{D}_{X}^{V} \nabla \alpha_{1}(Y) \\
& -2\left(\mathrm{D}_{Y}^{V} \mathrm{D}_{X}^{H}-\mathrm{D}_{\mathrm{D}_{Y}^{V}}^{H}\right) \alpha_{1}(\mathbf{T})-2 \mathrm{D}_{X}^{H} \alpha_{1}(Y)+6 \mathrm{D}_{Y}^{H} \nabla \alpha_{2}(X) \\
& -3 \mathrm{D}_{Y}^{V} \nabla^{2} \alpha_{2}(X)+2 \mathrm{D}_{X}^{V} \alpha_{2}(\Phi(Y))+2 \alpha^{2}\left(\mathrm{D}_{X}^{V} \Phi(Y)\right)+\mathrm{D}_{Y}^{V} \alpha_{2}(\Phi(X)) \\
& \left.+\alpha_{2}\left(\mathrm{D}_{Y}^{V} \Phi(X)\right)\right] .
\end{aligned}
$$


At last we can make use of some further simplifications which follow from the result of the preceding proposition. Indeed, from $\nabla \alpha_{2}(Y)=\mathrm{D}_{Y}^{V} \alpha_{1}(\mathbf{T})$ it follows that

$$
\begin{aligned}
& { }^{*} \nabla^{2} \alpha_{2}(\mathbf{T})=0 \\
& * \nabla^{2} \alpha_{2}(Y)=\mathrm{D}_{Y}^{V} \nabla \alpha_{1}(\mathbf{T})-\mathrm{D}_{Y}^{H} \alpha_{1}(\mathbf{T}) \\
& \text { * } \mathrm{D}_{X}^{V} \nabla^{2} \alpha_{2}(Y)=\left(\mathrm{D}_{X}^{V} \mathrm{D}_{Y}^{V}-\mathrm{D}_{\mathrm{D}_{X}^{V} Y}^{V}\right) \nabla \alpha_{1}(\mathbf{T})-\left(\mathrm{D}_{X}^{V} \mathrm{D}_{Y}^{H}-\mathrm{D}_{\mathrm{D}_{X}^{V} Y}^{H}\right) \alpha_{1}(\mathbf{T}) \\
& +\mathrm{D}_{Y}^{V} \nabla \alpha_{1}(X)-\mathrm{D}_{Y}^{H} \alpha_{1}(X) \\
& \text { * } \mathrm{D}_{X}^{H} \nabla \alpha_{2}(Y)=\left(\mathrm{D}_{X}^{H} \mathrm{D}_{Y}^{V}-\mathrm{D}_{\mathrm{D}_{X}^{H} Y}^{V}\right) \alpha_{1}(\mathbf{T})=\left(\mathrm{D}_{Y}^{V} \mathrm{D}_{X}^{H}-\mathrm{D}_{\mathrm{D}_{Y}^{V} X}^{H}\right) \alpha_{1}(\mathbf{T}) \text {. }
\end{aligned}
$$

So condition (5.32) becomes

$$
\begin{aligned}
0= & 3\left(\mathrm{D}_{X}^{V} \mathrm{D}_{Y}^{V}-\mathrm{D}_{\mathrm{D}_{X}^{V} Y}^{V}\right) \nabla \alpha_{1}(\mathbf{T})+\mathrm{D}_{Y}^{V} \nabla \alpha_{1}(X)-\mathrm{D}_{X}^{V} \nabla \alpha_{1}(Y) \\
& +2 \mathrm{D}_{X}^{V} \alpha_{2}(\Phi(Y))+2 \alpha_{2}\left(\mathrm{D}_{X}^{V} \Phi(Y)\right)-2 \mathrm{D}_{X}^{V} \nabla^{2} \alpha_{2}(Y) \\
& +\mathrm{D}_{Y}^{V} \alpha_{2}(\Phi(X))+\alpha_{2}\left(\mathrm{D}_{Y}^{V} \Phi(X)\right)-\mathrm{D}_{Y}^{V} \nabla^{2} \alpha_{2}(X) .
\end{aligned}
$$

Putting $X=\mathbf{T}$, we get

$$
\begin{aligned}
0 & =\mathrm{D}_{Y}^{V} \nabla \alpha_{1}(\mathbf{T})-\nabla \alpha_{1}(Y)+\alpha_{2}(\Phi(Y))-\nabla^{2} \alpha_{2}(Y) \\
& \Downarrow \\
\nabla \alpha_{1}(Y) & =\alpha_{2}(\Phi(Y))+\mathrm{D}_{Y}^{H} \alpha_{1}(\mathbf{T}) .
\end{aligned}
$$

Now we just have to prove that (5.28) is also a sufficient condition. Therefore we take $\mathrm{D}_{X}^{V}$ of (5.28) and we get

$$
\begin{aligned}
\mathrm{D}_{X}^{V} \nabla & \alpha_{1}(Y) \\
= & \left(\mathrm{D}_{X}^{V} \mathrm{D}_{Y}^{H}-\mathrm{D}_{\mathrm{D}_{X}^{V} Y}^{H}\right) \alpha_{1}(\mathbf{T})+\mathrm{D}_{Y}^{H} \alpha_{1}(X)+\mathrm{D}_{X}^{V} \alpha_{2}(\Phi(Y))+\alpha_{2}\left(\mathrm{D}_{X}^{V} \Phi(Y)\right) \\
= & \left(\mathrm{D}_{X}^{V} \mathrm{D}_{Y}^{V}-\mathrm{D}_{\mathrm{D}_{X}^{V} Y}^{V}\right) \nabla \alpha_{1}(\mathbf{T})+\mathrm{D}_{Y}^{V} \nabla \alpha_{1}(X)-\mathrm{D}_{X}^{V} \nabla^{2} \alpha_{2}(Y) \\
& +\mathrm{D}_{X}^{V} \alpha_{2}(\Phi(Y))+\alpha_{2}\left(\mathrm{D}_{X}^{V} \Phi(Y)\right)
\end{aligned}
$$

and if we compare this with (5.32), it is immediately clear that (5.28) is also sufficient.

Now, we will look at the interesting special situation that $\alpha=d f=\left(d^{V} f\right)^{V}+$ $\left(d^{H} f\right)^{H}$. In that case, condition (5.25) is automatically satisfied. Indeed, we have

$$
\begin{aligned}
\nabla \alpha_{2}(Y) & =\nabla d^{V} f(Y)=\left(d^{V} \nabla f-d^{H} f\right)(Y)=\left(d^{V}\left(d^{H} f(\mathbf{T})\right)-d^{H} f\right)(Y) \\
& =\mathrm{D}_{Y}^{V} d^{H} f(\mathbf{T})=\mathrm{D}_{Y}^{V} \alpha_{1}(\mathbf{T}) .
\end{aligned}
$$


Also condition (5.28) is automatically satisfied, namely

$$
\left.\nabla \alpha_{1}(Y)=\nabla \mathrm{D}_{Y}^{H} f-\mathrm{D}_{\nabla Y}^{H} f=\mathrm{D}_{Y}^{H} \nabla f+\mathrm{D}_{\Phi(Y)}^{V} f\right)=\mathrm{D}_{Y}^{H} \alpha_{1}(\mathbf{T})+\alpha_{2}(\Phi(Y)) .
$$

Note further that since $\nabla(\operatorname{tr} K)=\nabla \alpha_{1}(\mathbf{T})=\nabla f$, the function $f$ is the trace of the tensor $K$ (to within a first integral). If we assume that $K$ is symmetric, then follows from (5.12) that $d^{V} f$ has to be zero, in other words: the trace of $K$ has to be basic.

\subsection{A recursive scheme of first integrals}

We start from an arbitrary symmetric tensor field $K$ which satisfies the fundamental relation (5.22). So, we leave for a while the path of R-tensors and we do not assume that $K$ comes from a tensor $J$ or is homogeneous. Observe now that

$$
\begin{aligned}
\nabla(g(\mathbf{T}, K \mathbf{T})) & =g(\mathbf{T}, \nabla K(\mathbf{T}))=\frac{1}{2} g\left(\mathbf{T}, \alpha_{1}(\mathbf{T}) \mathbf{T}-2 E A_{1}\right), \\
& =\alpha_{1}(\mathbf{T}) E-E g\left(\mathbf{T}, A_{1}\right)=2 E \alpha_{1}(\mathbf{T})=2 E \nabla \operatorname{tr} K .
\end{aligned}
$$

We know of course that $E$ is a first integral of the geodesic spray $\Gamma$, and the above calculation now reveals that also $\frac{1}{2} g(\mathbf{T}, K \mathbf{T})-E \operatorname{tr} K$ is a first integral. In fact, we will show that there exists a whole hierarchy of (non-quadratic) first integrals.

Let us first define the functions

$$
k_{m}=\frac{1}{2} g\left(\mathbf{T}, K^{m} \mathbf{T}\right)
$$

and

$$
a_{m}=\frac{1}{m} \operatorname{tr} K^{m}
$$

for $m=1,2, \ldots$, and put $k_{0}=E$.

Lemma 9. Under the assumption (5.22), we have for $m \geq 1$ that

$$
\begin{aligned}
\nabla a_{m} & =\alpha_{1}\left(K^{m} \mathbf{T}\right), \\
\nabla k_{m} & =\sum_{i=1}^{m}\left(\nabla a_{i}\right) k_{m-i} .
\end{aligned}
$$


Proof. From (5.22) it follows, for example, that

$\nabla K^{2}=K \circ \nabla K+\nabla K \circ K=\frac{1}{2}\left(K \alpha_{1} \otimes \mathbf{T}+\alpha_{1} \otimes K \mathbf{T}-K \theta_{E} \otimes A_{1}-\theta_{E} \otimes K A_{1}\right)$ and it can be shown by induction that

$$
\nabla K^{m+1}=\frac{1}{2} \sum_{i=0}^{m}\left(K^{i} \alpha_{1} \otimes K^{m-i} \mathbf{T}-K^{i} \theta_{E} \otimes K^{m-i} A_{1}\right) .
$$

Taking a trace, (5.35) immediately follows. Moreover, since $\nabla g=0$ and $\nabla \mathbf{T}=0$, we have

$$
\nabla k_{m}=\frac{1}{2} g\left(\mathbf{T}, \nabla K^{m}(\mathbf{T})\right) .
$$

A direct computation, using the formula for $\nabla K^{m}$ just derived, leads to (5.36).

We next introduce auxiliary functions $\phi_{k}$, defined recursively by the following scheme

$$
\phi_{1}=0, \quad \phi_{k}=\frac{1}{2} \sum_{i=1}^{k-1} a_{i} a_{k-i}-\frac{1}{k} \sum_{i=2}^{k-1}(k-i) \phi_{i} a_{k-i}, \quad k \geq 2 .
$$

The dynamical covariant derivative of such a function $\phi_{j}$ is computed in the next lemma.

Lemma 10. $\quad \nabla \phi_{j}=\sum_{l=1}^{j-1} a_{l} \nabla a_{j-l}-\sum_{l=2}^{j-1} \phi_{l} \nabla a_{j-l}, \quad j \geq 2$.

Proof. We have $\phi_{2}=\frac{1}{2} a_{1}^{2}$ and $\nabla \phi_{2}=a_{1} \nabla a_{1}$ so that the property is true for $j=2$. Assume it is valid for all $j$ up to $k-1$ and now act with $\nabla$ on $\phi_{k}$ as defined by (5.37). We get

$$
\begin{aligned}
\nabla \phi_{k}=\sum_{i=1}^{k-1} a_{i} \nabla a_{k-i}-\frac{1}{k} \sum_{i=2}^{k-1}(k-i) \phi_{i} \nabla a_{k-i} \\
\quad-\frac{1}{k} \sum_{i=2}^{k-1}(k-i) a_{k-i} \sum_{l=1}^{i-1} a_{l} \nabla a_{i-l}+\frac{1}{k} \sum_{i=2}^{k-1}(k-i) a_{k-i} \sum_{l=2}^{i-1} \phi_{l} \nabla a_{i-l} .
\end{aligned}
$$

In order to collect coefficients of $\nabla a_{j}$, we put $j=k-i$ in the second term on the right and $j=i-l$ in the last two terms (and adjust the summations).

$$
\begin{aligned}
\nabla \phi_{k}=\sum_{i=1}^{k-1} a_{i} \nabla a_{k-i}-\frac{1}{k} \sum_{j=1}^{k-2} j \phi_{k-j} \nabla a_{j} \\
\quad-\frac{1}{k} \sum_{i=2}^{k-1}(k-i) a_{k-i} \sum_{j=1}^{i-1} a_{i-j} \nabla a_{j}+\frac{1}{k} \sum_{i=2}^{k-1}(k-i) a_{k-i} \sum_{j=1}^{i-2} \phi_{i-j} \nabla a_{j} .
\end{aligned}
$$


We subsequently interchange the two summations in those last two terms and split off one term to have the common upper bound $k-3$ for $j$. The result reads

$$
\begin{aligned}
\nabla \phi_{k}=\sum_{i=1}^{k-1} a_{i} \nabla a_{k-i}-\frac{1}{k} \sum_{j=1}^{k-2} j \phi_{k-j} \nabla a_{j}-\frac{1}{k} a_{1}^{2} \nabla a_{k-2} \\
\quad-\frac{1}{k} \sum_{j=1}^{k-3} \nabla a_{j}\left(\sum_{i=j+1}^{k-1}(k-i) a_{k-i} a_{i-j}-\sum_{i=j+2}^{k-1}(k-i) a_{k-i} \phi_{i-j}\right) .
\end{aligned}
$$

In the terms between brackets, we now change $i-j$ to $l$. We also split of the term for $j=k-3$ in the second summation of the equation. Because $\phi_{2}=\frac{1}{2} a_{1}^{2}$, we get

$$
\begin{aligned}
\nabla \phi_{k}= & \sum_{i=1}^{k-1} a_{i} \nabla a_{k-i}-\frac{1}{k} \sum_{j=1}^{k-3} j \phi_{k-j} \nabla a_{j}-\phi_{2} \nabla a_{k-2} \\
& -\frac{1}{k} \sum_{j=1}^{k-3} \nabla a_{j}\left(\sum_{l=1}^{k-j-1}(k-j-l) a_{k-j-l} a_{l}-\sum_{l=2}^{k-j-1}(k-j-l) a_{k-j-l} \phi_{l}\right) .
\end{aligned}
$$

Now we use the definition of $\phi_{k}$ (see (5.37)) to replace the terms between the brackets. The second summation of equation (5.38) will cancel out a part of it.

$$
\begin{aligned}
\nabla \phi_{k}= & \sum_{i=1}^{k-1} a_{i} \nabla a_{k-i}-\phi_{2} \nabla a_{k-2} \\
& \quad-\frac{1}{k} \sum_{j=1}^{k-3} \nabla a_{j}\left(k \phi_{k-j}+\sum_{l=1}^{k-j-1}\left(\frac{k-j}{2}-l\right) a_{k-j-l} a_{l}\right) .
\end{aligned}
$$

The second term between the brackets is zero. Indeed,

$$
\sum_{l=1}^{k-j-1}\left(\frac{k-j}{2}-l\right) a_{k-j-l} a_{l}=\sum_{l=1}^{k-j-1} a_{k-j-l} a_{l}\left(\frac{k-j-l}{2}+\frac{l}{2}-l\right)=0 .
$$

We get

$$
\nabla \phi_{k}=\sum_{i=1}^{k-1} a_{i} \nabla a_{k-i}-\sum_{j=1}^{k-2} \phi_{k-j} \nabla a_{j}=\sum_{i=1}^{k-1} a_{i} \nabla a_{k-i}-\sum_{i=2}^{k-1} \phi_{i} \nabla a_{k-i} .
$$


Consider now the functions $h_{m}$ defined by $h_{0}=E$ and

$$
h_{m}=k_{m}+\sum_{i=1}^{m} \rho_{i} k_{m-i}, \quad \text { with } \quad \rho_{k}=\phi_{k}-a_{k}, \quad m \geq 1 .
$$

There is also an alternative way to defines these functions, namely as

$$
h_{m}=k_{m}-\sum_{i=1}^{m} c_{i} h_{m-i}, \quad \text { with } \quad c_{k}=a_{k}+\psi_{k}, \quad m \geq 1
$$

where

$$
\psi_{1}=0, \quad \psi_{k}=\frac{1}{2} \sum_{i=1}^{k-1} a_{i} a_{k-i}+\frac{1}{k} \sum_{i=2}^{k-1}(k-i) \psi_{i} a_{k-i}, \quad k \geq 2 .
$$

Note that we have already seen that $h_{0}=E$ and $h_{1}=\frac{1}{2} g(\mathbf{T}, K \mathbf{T})-E \operatorname{tr} K$ are first integrals. The point is now that all the functions $h_{m}$ we have just defined, are first integrals.

Theorem 21. Let $K$ be a non-singular type $(1,1)$ tensor, symmetric with respect to a Finsler metric $g=\mathrm{D}^{V} \mathrm{D}^{V} E$. Assume that $K$ satisfies the requirement (5.22), then the functions $h_{m}$ (5.39) are first integrals of the geodesic spray of $g$ for all $m$.

Proof. From lemma 9 we have, using also $\nabla k_{0}=0$,

$$
\nabla h_{m}=\sum_{i=1}^{m}\left(\nabla \phi_{i}\right) k_{m-i}+\sum_{i=1}^{m-1} \rho_{i} \sum_{j=1}^{m-i}\left(\nabla a_{j}\right) k_{m-i-j} .
$$

Putting $k=i+j$ in the second term and interchanging the double summation, this becomes

$$
\begin{aligned}
\nabla h_{m} & =\sum_{i=1}^{m}\left(\nabla \phi_{i}\right) k_{m-i}+\sum_{i=1}^{m-1} \sum_{l=i+1}^{m}\left(\nabla a_{l-i}\right) k_{m-l} \\
& =\sum_{l=2}^{m}\left(\left(\nabla \phi_{l}\right)+\sum_{i=1}^{l-1} \rho_{i}\left(\nabla a_{l-i}\right)\right) k_{m-l}+\left(\nabla \phi_{1}\right) k_{m-1} .
\end{aligned}
$$

This is clearly zero in view of $\phi_{1}=0$ and lemma 10 . 
Remark that the functions $\rho_{k}$ are in fact the coefficients of the polynomial $\operatorname{det}(z I-K)=z^{n}+\rho_{1} z^{n-1}+\ldots+\rho_{n}\left(\rho_{1}=-\operatorname{tr} K, \rho_{2}=\frac{1}{2}\left(\operatorname{tr}(K)^{2}-\left(\operatorname{tr} K^{2}\right)\right)\right.$, $\left.\ldots, \rho_{n}=(-1)^{n} \operatorname{det} K\right)$. Note further that the first integrals $h_{m}$ are of the form $\frac{1}{2} g\left(\mathbf{T}, B_{(m)} \mathbf{T}\right)$ where $B_{(m)}$ is a type $(1,1)$ tensor field given by

$$
B_{(m)}=K^{m}+\sum_{i=1}^{m} \rho_{i} K^{m-i}=\rho_{m} I+K B_{(m-1)}
$$

with $B_{(0)}=I$. There is also a link between these tensors $B_{(m)}$ and the cofactor tensor of $K+s I$. Indeed, if $K$ satisfies (5.22), then so will $K+s I$. This implies that $g\left(A_{K+s I} \mathbf{T}, \mathbf{T}\right)$ with $A_{K+s I}=\sum_{m=1}^{n} A_{(m)} s^{m-1}$ the cofactor tensor of $K+s I$, is a first integral. Equating the coefficients of the different powers of $s$ in the defining relation:

$(K+s I) A_{K+s I}=\operatorname{det}(K+s I) I=\left(s^{n}-\rho_{1} s^{n-1}+\ldots+(-1)^{m} \rho_{m} s^{n-m}+(-1)^{n} \rho_{n}\right) I$,

we find that

$$
K A_{(m+1)}+A_{(m)}=(-1)^{n-m} \rho_{n-m} I \quad \text { with } A_{(n)}=I
$$

or

$$
(-1)^{m} A_{(n-m)}=\rho_{m} I+K(-1)^{m-1} A_{(n-m+1)} \quad \text { with } A_{(n)}=I .
$$

So, $B_{(m)}=(-1)^{m} A_{(n-m)}$ for $m=1, \ldots, n$. $A_{(1)}$ is the cofactor $A=$ $K^{-1} \operatorname{det} K$ of $K$ and we have just shown that $g(A \mathbf{T}, \mathbf{T})$ is a first integral. We can also proof this in another way. Namely, for a symmetric tensor $K$ which satisfies (5.22), we have that

$$
\begin{aligned}
\operatorname{tr}\left(K^{-1} \nabla K\right) & =\frac{1}{2}\left(\alpha_{1}\left(K^{-1} \mathbf{T}\right)-g\left(\mathbf{T}, K^{-1} A_{1} \mathbf{T}\right)\right) \\
& =\alpha_{1}\left(K^{-1} \mathbf{T}\right)
\end{aligned}
$$

So, we find that

$$
\begin{aligned}
& g(\nabla A(\mathbf{T}), \mathbf{T}) \\
& \quad=\operatorname{det} K\left(\operatorname{tr}\left(K^{-1} \nabla K\right) g\left(K^{-1} \mathbf{T}, \mathbf{T}\right)-g\left(\nabla K\left(K^{-1} \mathbf{T}\right), K^{-1} \mathbf{T}\right)\right) \\
& \quad=(\operatorname{det} K) g\left(K^{-1} \mathbf{T}, \mathbf{T}\right)\left(\operatorname{tr}\left(K^{-1} \nabla K\right)-\alpha_{1}\left(K^{-1} \mathbf{T}\right)\right)=0
\end{aligned}
$$

where we made use of the general property

$$
D(\log \operatorname{det} M)=\operatorname{tr}\left(M^{-1} D M\right)
$$

which hold for any matrix $M$ and differentiation operator $D$. 
Remark further that $A_{(1)}=A$ is equal to $(-1)^{n-1} B_{(n-1)}$. So,

$$
B_{(n)}=\rho_{n} I+K B_{(n-1)}=(-1)^{n}(\operatorname{det} K) I+(-1)^{n-1} K A=0 .
$$

Finally, we come back to the case that the tensor $K$ is related to an R-tensor. So, we consider now a symmetric type $(1,1)$ tensor field $K$ homogeneous of degree 0 which satisfies the conditions (4.9). We also assume that $N_{K}^{V}=0$ (which is needed for $N_{R}$ to vanish). As shown above we have a whole hierarchy of first integrals $h_{m}$. But in this situation we can also find a recursive formula for the functions $k_{m}$ (5.33) which define these first integrals.

The assumption $N_{K}^{V}=0$ implies that $\left(d^{V}, d_{K}^{V}\right)$ forms a bi-differential calculus. Indeed, it implies that also $\mathcal{N}_{K}^{V}=0$ which is equivalent to $d_{K}^{V 2}=0$. Further we have that $d^{V 2}=0$ and $d^{V} d_{K}^{V}+d_{K}^{V} d^{V}=0$. So, if there exist a function $\chi^{(0)}$ satisfying $d^{V} d_{K}^{V} \chi^{(0)}=0$, we know that there is a sequence of functions determined by

$$
d^{V} \chi^{(i+1)}=d_{K}^{V} \chi^{(i)} .
$$

In the following proposition we will show that this rule is true for $\chi^{(i)}=k_{i}$.

Proposition 32. Suppose that $K$ is a symmetric type $(1,1)$ tensor field, homogeneous of degree 0. Under the assumptions (4.9) and $N_{K}^{V}=0$, we have that $d^{V}\left(K \theta_{E}\right)=0$ or equivalently that $k_{0}=E$ satisfies the equation $d^{v} d_{K}^{V} k_{0}=0$. Moreover, we have

$$
d^{V} k_{m}=d_{K}^{V} k_{m-1}, \quad \text { where } \quad k_{m}=\frac{1}{2} g\left(\mathbf{T}, K^{m} \mathbf{T}\right) .
$$

Proof. We have

$$
\begin{aligned}
& d^{V}\left(K \theta_{E}\right)(X, Y) \\
& \quad=\mathrm{D}_{X}^{V} K\left(\theta_{E}\right)(Y)-\mathrm{D}_{Y}^{V} K\left(\theta_{E}\right)(X)+K\left(\mathrm{D}_{X}^{V} \theta_{E}\right)(Y)-K\left(\mathrm{D}_{Y}^{V} \theta_{E}\right)(X), \\
& \quad=g\left(\mathbf{T}, \mathrm{D}_{X}^{V} K(Y)\right)-g\left(\mathbf{T}, \mathrm{D}_{Y}^{V} K(X)\right)=0,
\end{aligned}
$$

in view of (4.9) and the homogeneity of $K$. Since $\theta_{E}=d^{V} E$, this is the same as saying that $d^{V} d_{K}^{V} E=0$.

For the second part, we proceed by induction:

$$
\begin{aligned}
d^{V} k_{m}(X) & =\frac{1}{2} \mathrm{D}_{X}^{V}\left(g\left(\mathbf{T}, K^{m} \mathbf{T}\right)\right)=g\left(K^{m} X, \mathbf{T}\right)+\frac{1}{2} g\left(\mathbf{T}, \mathrm{D}_{X}^{V} K^{m}(\mathbf{T})\right) \\
& =g\left(K^{m} X, \mathbf{T}\right)+\frac{1}{2} g\left(K \mathbf{T}, \mathrm{D}_{X}^{V} K^{m-1}(\mathbf{T})\right) \\
& =g\left(K^{m} X, \mathbf{T}\right)+\frac{1}{2} g\left(X, \mathrm{D}_{K \mathbf{T}}^{V} K^{m-1}(\mathbf{T})\right)
\end{aligned}
$$


Now $N_{K}^{V}=0$ implies that $\mathrm{D}_{K \mathbf{T}}^{V} K=K \circ \mathrm{D}_{\mathbf{T}}^{V} K=0$ and then $\mathrm{D}_{K \mathbf{T}}^{V} K^{m}=0$ by induction. Hence,

$$
d^{V} k_{m}(X)=K^{m} \theta_{E}(X)=K^{m-1} \theta_{E}(K X)=d^{V} k_{m-1}(K X)=d_{K}^{V} k_{m-1}(X),
$$

which gives the desired recursion.

Remark that if $N_{K}^{V}=0$, we also have for the functions $a_{m}$ that

$$
d^{V} a_{m}=d_{K}^{V} a_{m-1}
$$




\section{Chapter 6}

\section{Projective equivalence of Finsler metrics}

As we have seen in section 2.6, special conformal Killing tensors play a distinctive role in the study of projectively equivalent Riemannian metrics. It is therefore interesting to investigate whether the generalised notion of such tensors which we have encountered in the preceding chapter, could also be related to projective equivalence of geodesics of some kind. Most of our attention in this chapter will go to projective equivalence of geodesics associated to Finsler metrics. But it is perhaps a good question to ask under what circumstances one can talk about a notion of geodesics in the first place.

In a paper on the inverse problem of the calculus of variations for Finsler structures, Krupka and Sattarov [25] have introduced a concept of geodesics in a more general context than that of linear connections or more specifically Riemannian or Finsler metrics. Briefly, what they discussed is the following. Consider the fibre bundle $\Gamma Q$ of linear connections on a manifold $Q$, whose typical fibre has coordinates, $\gamma_{j k}^{i}$ say, which transform in the appropriate way. What they call a 'connection on $T Q$ ' then is a morphism $\mathcal{D}: T Q \rightarrow \Gamma Q$ over the identity on $Q$. It is claimed that there is an associated covariant derivative operator which, for example on morphisms $g: T Q \rightarrow \mathcal{T}_{2}^{0} Q$ over $\mathrm{id}_{Q}$, is defined by the coordinate formula

$$
g_{i j ; k}=\frac{\partial g_{i j}}{\partial q^{k}}-\frac{\partial g_{i j}}{\partial \dot{q}^{s}} \gamma_{r k}^{s} \dot{q}^{r}-g_{i m} \gamma_{j k}^{m}-g_{j m} \gamma_{i k}^{m} .
$$

A geodesic of such a connection is said to be a curve in $Q$ which satisfies the differential equations

$$
\dot{q}^{k}+\gamma_{i j}^{k}(q, \dot{q}) \dot{q}^{i} \dot{q}^{j}=0 .
$$

It is clear that the kind of metric tensor fields $g$ one is talking about here are metrics along the tangent bundle projection $\tau: T Q \rightarrow Q$ and that the 
connection under consideration would better be called 'connection along the tangent bundle projection' as well.

We take the opportunity to make an excursion into this field in the next section, with the specific purpose of justifying the constructions just mentioned by a more coordinate free approach.

\subsection{Linear connection along the tangent bundle projection}

Let $\Gamma Q \rightarrow Q$ be the fibre bundle of linear connections on $Q$ and consider a map $\mathcal{D}: T Q \rightarrow \Gamma Q$ which makes the following diagram commutative:

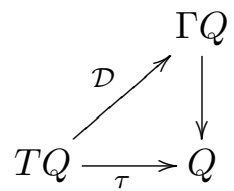

We have two principal objectives now: (i) show how one can come from such a $\mathcal{D}$, in an intrinsic way, to a notion of covariant derivative which justifies (6.1); (ii) develop at the same time a parallel transport along curves in $Q$, which generalises the usual constructions for a linear connection, and leads to the equations (6.2) as 'geodesic equations'.

The fibre of $\Gamma Q$ at a point $m \in Q$ consist of maps $D_{m}: T_{m} Q \times \mathcal{X}_{m} \rightarrow T_{m} Q$, where $\mathcal{X}_{m}$ denotes the module of vector fields on $Q$ defined in a neighbourhood of $m$, with properties (omitting the subscript $m$ in the notation)

$$
\begin{aligned}
D_{\lambda v_{m}} Y & =\lambda D_{v_{m}} Y, \quad \lambda \in \mathbb{R} \\
D_{v_{m}}(f Y) & =f(m) D_{v_{m}} Y+v_{m}(f)(m) Y(m), \quad f \in C^{\infty}(Q)
\end{aligned}
$$

plus linearity with respect to the sum in both arguments. It follows for the map $\mathcal{D}: T Q \rightarrow \Gamma Q$ under consideration, that $\mathcal{D}\left(w_{m}\right)$ is a map from $T_{m} Q \times \mathcal{X}_{m}$ to $T_{m} Q$ of this kind, which is determined by locally defined functions $\gamma_{i j}^{k}$ on $T Q$, such that

$$
\left.\mathcal{D}\left(w_{m}\right)_{\frac{\partial}{\partial q^{i}}}\right|_{m} \frac{\partial}{\partial q^{j}}=\left.\gamma_{i j}^{k}\left(w_{m}\right) \frac{\partial}{\partial q^{k}}\right|_{m} .
$$

These pointwise properties can be extended in a natural way. Consider a map

$$
D: \mathcal{X}(\tau) \times \mathcal{X}(Q) \rightarrow \mathcal{X}(\tau)
$$


defined for all $X \in \mathcal{X}(\tau), Y \in \mathcal{X}(Q)$ by

$$
\left(D_{X} Y\right)\left(w_{m}\right)=\mathcal{D}\left(w_{m}\right)_{X_{w_{m}}} Y,
$$

which will have the properties

$$
\begin{aligned}
D_{F X} Y & =F D_{X} Y, \quad \forall F \in C^{\infty}(T Q) \\
D_{X}(f Y) & =f D_{X} Y+X(f) Y, \quad \forall f \in C^{\infty}(Q),
\end{aligned}
$$

where $Y \in \mathcal{X}(Q)$ is regarded as element of $\mathcal{X}(\tau)$ in the right-hand side of the second relation. We have of course also the linearity properties:

$$
D_{X_{1}+X_{2}} Y=D_{X_{1}} Y+D_{X_{2}} Y, \quad D_{X}\left(Y_{1}+Y_{2}\right)=D_{X} Y_{1}+D_{X} Y_{2} .
$$

Conversely, every operator $D: \mathcal{X}(\tau) \times \mathcal{X}(Q) \rightarrow \mathcal{X}(\tau)$ with the above four properties determines a linear connection along $\tau$, in the sense of the commutative diagram we started from. Indeed, the value of $D_{X} Y$ at any point $w_{m} \in T_{m} Q$ depends in the argument $X$ only on the value of $X$ at $w_{m}$. This can be shown by using a standard argument with a bump function on $T Q$ which is zero outside a neighbourhood of $w_{m}$. As a result, it makes sense to put

$$
\mathcal{D}\left(w_{m}\right)_{v_{m}} Y=\left(D_{X} Y\right)\left(w_{m}\right),
$$

where $X$ is any vector field along $\tau$ such that $X_{w_{m}}=v_{m}$. It then easily follows that $\mathcal{D}\left(w_{m}\right)$ is a map which has all the properties for belonging to the fibre of $\Gamma Q$ at $m$.

It is not yet clear how the operator $D: \mathcal{X}(\tau) \times \mathcal{X}(Q) \rightarrow \mathcal{X}(\tau)$ could be extended to a covariant derivation for arbitrary tensor fields along $\tau$, because the second argument is restricted to vector fields on $Q$ so far. A suitable notion of horizontal lift will help us around this difficulty.

Let $\sigma: t \mapsto q^{i}(t)$ be a curve in $Q$ and consider a vector field along $\sigma$, i.e. a curve $\eta: t \mapsto\left(q^{i}(t), \eta^{i}(t)\right)$ in $T Q$ which projects into $\sigma$. We can now construct another vector field $D_{\dot{\sigma}} \eta$ along $\sigma$, defined for each value of $t$ by

$$
\left(D_{\dot{\sigma}} \eta\right)(t)=\mathcal{D}(\eta(t))_{\dot{\sigma}(t)} Y,
$$

where $Y$ is any vector field on $Q$ such that $Y(\sigma(t))=\eta(t)$ in some open neighbourhood. In coordinates, we have

$$
D_{\dot{\sigma}} \eta(t)=\left.\left(\dot{\eta}^{k}(t)+\gamma_{i j}^{k}(\eta(t)) \dot{q}^{i}(t) \eta^{j}(t)\right) \frac{\partial}{\partial q^{k}}\right|_{\sigma(t)},
$$


where $t \mapsto\left(q^{i}(t), \dot{q}^{i}(t)\right)$ is the coordinate representation of the curve $\dot{\sigma}$ in $T Q$ and $\dot{\eta}^{k}$ stands for

$$
\dot{\eta}^{k}(t)=\frac{\partial Y^{k}}{\partial q^{i}}(q(t)) \dot{q}^{i}(t)
$$

It is now possible to associate a concept of parallel transport along $\sigma$ to $D$. If $D_{\dot{\sigma}} \eta(t)=0$ for all $t$, then $\eta$ is called parallel along $\sigma$. Alternatively, we say that $\eta$ is a horizontal lift $\sigma^{h}$ of $\sigma$, determined by the given linear connection along $\tau$. As in the standard theory, it follows from the existence and uniqueness of solutions of first order ODEs, that for a given curve $\sigma$ in $Q$ and an arbitrary point $v$ in the fibre of $\sigma\left(t_{0}\right)$ say, there is an unique horizontal curve $\sigma^{h}$ passing through $v$ and projecting onto $\sigma$. The differential equations to be solved for the $\eta^{i}(t)$ are of course non-linear here.

We can now properly define geodesics of a given linear connection along $\tau$, as being curves $\gamma$ in $Q$ which satisfy

$$
D_{\dot{\gamma}} \dot{\gamma}=0 .
$$

It is clear that these are indeed curves $t \mapsto q^{i}(t)$ which are solutions of the second order differential equations (6.2).

If $X$ is a vector field on $Q$, we define its horizontal lift $X^{h}$ as being the vector field on $T Q$ which projects onto $X$ and is further being determined by the requirement that its integral curves are horizontal lifts of integral curves of $X$. It follows from what precedes that $X^{h}$ has a coordinate appearance of the form

$$
X^{h}=X^{k}(q) \frac{\partial}{\partial q^{k}}-\gamma_{i j}^{k}(q, u) X^{i}(q) u^{j} \frac{\partial}{\partial u^{k}} .
$$

The horizontal lift can be extended to an operator from $\mathcal{X}(\tau)$ to $\mathcal{X}(T Q)$ by imposing $C^{\infty}(T Q)$-linearity. This brings us in the position to extend the domain of the operator $D$ even further, namely to an operator

$$
D: \mathcal{X}(\tau) \times \mathcal{X}(\tau) \rightarrow \mathcal{X}(\tau) .
$$

Indeed, since the module $\mathcal{X}(\tau)$ is finitely generated over $C^{\infty}(T Q)$ by $\mathcal{X}(Q)$, it suffices to put

$$
D_{X}(F Y)=F D_{X} Y+X^{h}(F) Y \quad \text { for } Y \in \mathcal{X}(Q) \text { and } F \in C^{\infty}(T Q) .
$$

It is then natural to define the action of $D_{X}$ on functions, simply by

$$
D_{X} F=X^{h}(F .)
$$


Having done that, $D_{X}$ immediately extends to 1 -forms along $\tau$ by duality, and subsequently to tensor fields along $\tau$ of arbitrary covariant and contravariant order. In particular, we will have for $g \in \mathcal{T}_{2}^{0}(\tau)$, that $D_{X} g$ is defined by

$$
\left(D_{X} g\right)(Y, Z)=X^{h}(g(Y, Z))-g\left(D_{X} Y, Z\right)-g\left(Y, D_{X} Z\right) .
$$

This justifies the covariant derivative formula (6.1), which was put forward in $[25]$.

For the rest of this section, $\Gamma$ will denote the SODE field of the geodesic equations (6.2). It is interesting to observe that

$$
\mathbf{T}^{h}=\Gamma \quad \text { and } \quad D_{\mathbf{T}} \mathbf{T}=0 .
$$

There are a number of other interesting features which may be worth a deeper investigation in the future. A striking point, for example, is that the standard SODE-connection, associated to $\Gamma$, is not the connection $D$ we started from. To simplify this discussion a bit, since only the symmetric components of the $\gamma_{i j}^{k}$ play a role for the geodesics (6.2), let us assume that

$$
\gamma_{i j}^{k}=\gamma_{j i}^{k} .
$$

The connection coefficients of the non-linear SODE-connection associated to $\Gamma$ the are given by

$$
\Gamma_{i}^{k}=\gamma_{i j}^{k} u^{j}+\frac{1}{2} \frac{\partial \gamma_{l m}^{k}}{\partial u^{i}} u^{l} u^{m}
$$

In particular, $\Gamma_{i j}^{k}=\frac{\partial \Gamma_{i}^{k}}{\partial u^{j}}$ is different from $\gamma_{i j}^{k}$. We have a different lift operator also,

$$
X \mapsto X^{H}=X^{i}\left(\frac{\partial}{\partial q^{i}}-\Gamma_{i}^{k} \frac{\partial}{\partial u^{k}}\right) \neq X^{h},
$$

and $\mathbf{T}^{H}$ is not, in general, equal to $\Gamma$. Furthermore, both $\mathrm{D}_{\mathbf{T}}^{H}$ and the dynamical covariant derivative $\nabla$, which plays such an important role in many of our considerations, are not the same as the operator $D_{\mathbf{T}}$. As a matter of fact we have

$$
\mathrm{D}_{\mathbf{T}}^{H} \frac{\partial}{\partial q^{i}}=u^{j} \Gamma_{i j}^{k} \frac{\partial}{\partial q^{k}} \quad \text { and } \quad \nabla \frac{\partial}{\partial q^{i}}=\Gamma_{i}^{k} \frac{\partial}{\partial q^{k}} \quad \text { while } \quad D_{\mathbf{T}} \frac{\partial}{\partial q^{i}}=u^{j} \gamma_{j i}^{k} \frac{\partial}{\partial q^{k}} \text {. }
$$

In general $\mathrm{D}_{\mathbf{T}}^{H} \mathbf{T}$ and $\nabla \mathbf{T}$ are not zero. 
Have we now seen the most general situation in which it is justified to call second-order equations geodesic equations? It should be said that Martinez has discussed in detail in his thesis [35] how one can introduce a notion of parallel transport also for arbitrary second-order equations. The problem there, however, (which is somewhat related to the remarks just made) is that when curves whose tangents are parallel, are called geodesics again, one ends up with geodesics equations which are different from the equations one started from. In fact, they are the equations associated to the secondorder equation field $\mathbf{T}^{H}$ which is commonly called the 'associated semispray' (see for example [13]). It is well known that $\mathbf{T}^{H}$ does coincide with the SODE one starts from, in the special case of a spray, i.e. a second-order differential equation field on $\mathcal{T} Q$ which is homogeneous of degree 2 . So, let us now come to the particular case of a spray, as seen within the context of linear connections along $\tau$ discussed in this section.

Going back to the commutative diagram of the beginning of this section, we now restrict the domain of $D$ to the slit tangent bundle $\mathcal{T} Q$ and assume that the map $\mathcal{D}: \mathcal{T} Q \rightarrow \Gamma Q$ has the homogeneity property

$$
\mathcal{D}\left(\lambda w_{m}\right)=\lambda \mathcal{D}\left(w_{m}\right) \quad \lambda \in \mathbb{R}(\neq 0) .
$$

This implies that the connection coefficients $\gamma_{i j}^{k}$ which we assume to be symmetric, are homogeneous functions of degree zero of the velocity coordinates. Hence, the geodesic equations (6.2) have 'force-terms' which are homogeneous of degree 2 and thus construct a spray. Conversely, let $\Gamma$ be a spray, for which the conventional coordinate representation is:

$$
\Gamma=u^{i} \frac{\partial}{\partial q^{i}}-2 \Gamma^{i} \frac{\partial}{\partial u^{i}}
$$

with coefficients $\Gamma^{i}$ which are homogeneous of degree 2. We thus have

$$
\frac{\partial \Gamma^{i}}{\partial u^{k}} u^{k}=2 \Gamma^{i}, \quad \text { and also } \quad \frac{\partial^{2} \Gamma^{i}}{\partial u^{k} \partial u^{l}} u^{k} u^{l}=2 \Gamma^{i} .
$$

To fit this within the frame work of linear connections along $\tau$, it suffices to define connection coefficients $\gamma_{i j}^{k}$ for such connection. Since the coefficients $\frac{\partial^{2} \Gamma^{i}}{\partial u^{k} \partial u^{l}}$ transform in the appropriate way, we can set

$$
\gamma_{i j}^{k}=\frac{\partial^{2} \Gamma^{k}}{\partial u^{i} \partial u^{j}}
$$


But observe on the other hand that if a homogeneous $\mathcal{D}$ is the starting point and we put $2 \Gamma^{i}=\gamma_{k l}^{i} u^{k} u^{l}$, it follows generally:

$$
\frac{\partial^{2} \Gamma^{i}}{\partial u^{k} \partial u^{l}}=\gamma_{k l}^{i}+\left(\frac{\partial \gamma_{k s}^{i}}{\partial u^{l}}+\frac{\partial \gamma_{l s}^{i}}{\partial u^{k}}\right) u^{s}+\frac{1}{2} \frac{\partial^{2} \gamma_{r s}^{i}}{\partial u^{k} \partial u^{l}} u^{r} u^{s} .
$$

This indicates that there is no unique identification between sprays and homogeneous linear connection along $\tau$. Also noteworthy is the fact that even in the case of a spray, the SODE-connection does not coincide necessarily with the connection along $\tau$, since the homogeneity properties do not help to simplify or reduce the relation (6.8). All such aspects may be worth further investigation. We limit ourselves here to the remark that at least the following features now better fit together. We have

$$
\mathbf{T}^{h}=\mathbf{T}^{H}=\Gamma
$$

and even though $\nabla=\mathrm{D}_{\mathbf{T}}^{H}$ still doesn't need to coincide with $D_{\mathbf{T}}$, we do have that $\nabla \mathbf{T}=0$ as well in the case of a spray.

We conclude this side exploration by the following remarks. Having recognised an intrinsic geometric meaning in equations of type (6.2) as geodesics, we could start studying projective equivalence of geodesics at this level of generality. It turns out, however, that this doesn't lead to any kind of elegant structure. In the next section, we directly pass to projective equivalence of sprays. There might be some advantages then in carrying out the subsequent investigations with the aid of the horizontal lift $X^{h}$ rather than $X^{H}$, or the covariant derivative operator $D_{\mathbf{T}}$ rather than $\nabla$. But for the sake of uniformity with the preceding chapters, we will stick to the familiar operations associated to the SODE-connection.

\subsection{Projective equivalence of sprays}

Suppose that we have a spray space $(Q, \Gamma)$ of dimension $n$ where $\Gamma$ is given by

$$
\Gamma=u^{i} \frac{\partial}{\partial q^{i}}-2 \Gamma^{i} \frac{\partial}{\partial u^{i}}
$$

with coefficients $\Gamma^{i}$ homogeneous of degree 2 in the velocities $u^{i}$. Consider also another spray $\widetilde{\Gamma}$ with spray coefficients $\widetilde{\Gamma}^{i}$.

Definition 24. Two sprays $\Gamma$ and $\widetilde{\Gamma}$ on a manifold are projectively (or geodesically) equivalent if their geodesics are the same up to a reparametrisation. 
This means that for any geodesic $c: I \subset \mathbb{R} \rightarrow M$ of $\Gamma$ there exists a parameter transformation $\sigma: \widetilde{I} \rightarrow I$ such that $\widetilde{c}:=c \circ \sigma$ is a geodesic of $\widetilde{\Gamma}$ and vice versa. It is also known that the sprays are projectively equivalent if and only if there exists a function $\lambda$ on $\mathcal{T} Q$, homogeneous of degree 1 such that

$$
\widetilde{\Gamma}=\Gamma-2 \lambda \Delta \quad \text { or } \quad \widetilde{\Gamma}^{i}=\Gamma^{i}+\lambda u^{i} .
$$

In terms of their non-linear connection coefficients, this relation is

$$
\widetilde{\Gamma}_{j}^{i}=\Gamma_{j}^{i}+u^{i} \frac{\partial \lambda}{\partial u^{j}}+\lambda \delta_{j}^{i}
$$

from which we obtain, after summing over $i$ and $j$ and using the homogeneity, the following formula for $\lambda$ :

$$
\lambda=\frac{1}{n+1}\left(\widetilde{\Gamma}_{i}^{i}-\Gamma_{i}^{i}\right)
$$

Another important result that we will need later, establishes a relation between the dynamical covariant derivatives of both sprays.

Proposition 33. Let $\Gamma$ and $\widetilde{\Gamma}=\Gamma-2 \lambda \Delta$ be projectively equivalent sprays, then

$$
\widetilde{\nabla}=\nabla-2 \lambda \mathrm{D}_{\mathbf{T}}^{V}+\mu_{A},
$$

where $A$ is the type $(1,1)$ tensor $d^{V} \lambda \otimes \mathbf{T}+\lambda I$.

Proof. The proof is a straightforward computation. For $F \in C^{\infty}(T Q)$ we have

$$
\widetilde{\nabla} F=\widetilde{\Gamma}(F)=(\Gamma-2 \lambda \Delta)(F)=\nabla F-2 \lambda \mathrm{D}_{\mathbf{T}}^{V} F .
$$

Next, for $X \in \mathcal{X}(\tau)$, the definition of the dynamical covariant derivative gives

$$
\begin{aligned}
\widetilde{\nabla} X & =\left(\widetilde{\Gamma}\left(X^{i}\right)+\widetilde{\Gamma}_{j}^{i} X^{j}\right) \frac{\partial}{\partial q^{i}} \\
& =\left(\Gamma\left(X^{i}\right)-2 \lambda \Delta\left(X^{i}\right)+\left(\Gamma_{j}^{i}+\frac{\partial \lambda}{\partial u^{j}} u^{i}+\lambda \delta_{j}^{i}\right) X^{j}\right) \frac{\partial}{\partial q^{i}} \\
& =\nabla X-2 \lambda \mathrm{D}_{\mathbf{T}}^{V} X+\lambda X+\left(d^{V} \lambda \otimes \mathbf{T}\right) X \\
& =\nabla X-2 \lambda \mathrm{D}_{\mathbf{T}}^{V} X+\mu_{\left(d^{V} \lambda \otimes \mathbf{T}+\lambda I\right)} X .
\end{aligned}
$$

For 1-forms $\alpha \in \mathcal{X}^{*}(\tau)$, the result follows by duality.

For more information on the projective equivalence of sprays, we refer to [45]. 


\subsection{Projective equivalence with the canonical spray on a Finsler space}

In this subsection we discuss the projective equivalence of two sprays in the particular case that one of the sprays is the canonical spray of a Finsler metric, also sometimes called the Finsler metrizability in a broad sense (see for example [48]). The proofs are similar to the one of lemma 8 in chapter 5 .

So, suppose that we have a Finsler manifold $(Q, E)$ with canonical spray $\Gamma$. We want to find the necessary and sufficient conditions for a spray $\widetilde{\Gamma}$ to be projectively equivalent to $\Gamma$.

If $\widetilde{\Gamma}=\Gamma-2 \lambda \Delta$, then (5.6) can be written, in view of the homogeneity of $E$, in the form

$$
\widetilde{\Gamma}\left(\frac{\partial E}{\partial u^{i}}\right)-\frac{\partial E}{\partial q^{i}}=-2 \lambda \frac{\partial E}{\partial u^{i}} .
$$

Conversely, if (6.15) holds for some $\lambda$ homogeneous of degree 1 and $\Gamma$ is the Euler-Lagrange field of $E$, it follows using the homogeneity of $E$ that

$$
\left(\Gamma^{k}-\widetilde{\Gamma}^{k}\right) \frac{\partial^{2} E}{\partial u^{k} \partial u^{i}}=-\lambda u^{k} \frac{\partial^{2} E}{\partial u^{k} \partial u^{i}},
$$

and then from the non-degeneracy of $E$ that $\widetilde{\Gamma}=\Gamma-2 \lambda \Delta$.

$\Gamma(E)=0$ implies for a projectively equivalent $\widetilde{\Gamma}$ that $\widetilde{\Gamma}(E)=-4 \lambda E$. Then, interchanging as before the partial derivatives in the first term of (6.15), we get

$$
\widetilde{H}_{i}(E)=-2 \frac{\partial \lambda}{\partial u^{i}} E-\lambda \frac{\partial E}{\partial u^{i}}
$$

This is called 'Rapcsak's equation' and can equivalently be written as

$$
d^{\widetilde{H}} E=-2 E d^{V} \lambda-\lambda d^{V} E .
$$

Conversely, (6.16) implies by applying it to $\mathbf{T}$ that $\widetilde{\Gamma}(E)=-4 \lambda E$. Using again the commutator of $\Gamma$ and $\frac{\partial}{\partial u^{i}}$, we see that (6.16) is identical to (6.15). Finally, using (6.14), it follows that $\nabla g=0$ implies

$$
\widetilde{\nabla} g=\mu_{A} g=-d^{V} \lambda \otimes d^{V} E-d^{V} E \otimes d^{V} \lambda-2 \lambda g .
$$

Conversely, following the method of proof of lemma 8 again, if (6.17) holds, the computation of $\widetilde{\nabla} g(\mathbf{T}, \mathbf{T})$ implies that $\widetilde{\Gamma}(E)=-4 \lambda E$ and then computing $\widetilde{\nabla} g(\mathbf{T}, X)$ leads to $(6.16)$.

So, to summarise, we have proven the following theorem. 
Theorem 22. A spray $\widetilde{\Gamma}$ on a Finsler manifold with energy function $E$ is projectively equivalent with the canonical spray $\Gamma$ if and only if there exists a function $\lambda$, homogeneous of degree 1, such that one of the following three equivalent conditions holds (and therefore all):

a) $\widetilde{\Gamma}\left(\frac{\partial E}{\partial u^{i}}\right)-\frac{\partial E}{\partial q^{i}}=-2 \lambda \frac{\partial E}{\partial u^{i}}$;

b) $d^{\widetilde{H}} E=-2 E d^{V} \lambda-\lambda d^{V} E$;

c) $\widetilde{\nabla} g=-d^{V} \lambda \otimes d^{V} E-d^{V} E \otimes d^{V} \lambda-2 \lambda g$.

\subsection{Projective equivalence of two Finsler metrics}

We shall start from a non-singular type $(1,1)$ tensor field $L$ along $\tau$, symmetric with respect to a given Finsler metric $g=\mathrm{D}^{V} \mathrm{D}^{V} E$ and homogeneous of degree 0 in the velocities. As already said before, we want to study in this section under what circumstances this tensor $L$ gives rise to a projectively equivalent metric $\widetilde{g}$ in exactly the same way as in the Riemannian case that we described in section 2.6.

So, consider the symmetric type $(0,2)$ tensor $\widetilde{g}$, homogeneous of degree 0 , defined by

$$
\widetilde{g}(X, Y)=(\operatorname{det} L)^{-1} g\left(L^{-1} X, Y\right) .
$$

In coordinates it is given by

$$
\widetilde{g}_{i j}=(\operatorname{det} L)^{-1} L^{-1}{ }_{i j},
$$

from which one can derive that

$$
\operatorname{det} \widetilde{g}=(\operatorname{det} L)^{-(n+1)} \operatorname{det} g .
$$

Conversely, one can use the above relations to define a type $(1,1)$ tensor $L$ for any two metric tensor fields (homogeneous of degree 0 , say, and assumed to be non-singular here).

Unlike in section 2.6 where the metric defined by (2.7) is immediately a Riemannian one, we have to impose an extra condition on $L$ for $\widetilde{g}$ defined by $(6.18)$ to be a Finsler metric. 
Proposition 34. $\widetilde{g}$ is a Finsler metric, i.e. $\widetilde{g}=\mathrm{D}^{V} \mathrm{D}^{V} \widetilde{E}$ for some $\widetilde{E}$ (homogeneous of degree 2), if and only if

$$
\begin{aligned}
& \operatorname{tr}\left(L \mathrm{D}_{Z}^{V} L^{-1}\right) g\left(L^{-1} X, Y\right)+g\left(\left(\mathrm{D}_{Z}^{V} L^{-1}\right) X, Y\right) \\
& \quad=\operatorname{tr}\left(L \mathrm{D}_{Y}^{V} L^{-1}\right) g\left(L^{-1} X, Z\right)+g\left(\left(\mathrm{D}_{Y}^{V} L^{-1}\right) X, Z\right),
\end{aligned}
$$

for all $X, Y, Z \in \mathcal{X}(\tau)$.

Proof. $\widetilde{g}$ is the Hessian of a function $\widetilde{E}$ if and only if it satisfies the Helmholtz condition $\left(\mathrm{D}_{Z}^{V} \widetilde{g}\right)(X, Y)=\left(\mathrm{D}_{Y}^{V} \widetilde{g}\right)(X, Z)$. From the definition of $\widetilde{g}$, the fact that $g$ is a Hessian, and the general property 5.43, one easily finds that this is equivalent to $(6.20)$.

The function $\widetilde{E}$ is not uniquely determined, but in view of the homogeneity of $\widetilde{g}$, it can be taken to be homogeneous of degree 2 and then necessarily must be

$$
\widetilde{E}=\frac{1}{2} \widetilde{g}(\mathbf{T}, \mathbf{T})=\frac{1}{2}(\operatorname{det} L)^{-1} g\left(L^{-1} \mathbf{T}, \mathbf{T}\right)
$$

The next step is to examine when the canonical spray $\widetilde{\Gamma}$, associated to this Finsler energy $\widetilde{E}$ is projectively equivalent with the original canonical spray $\Gamma$.

Theorem 23. Let $(Q, E)$ be a Finsler manifold, $L$ a non-singular type $(1,1)$ tensor, homogeneous of degree 0 and symmetric with respect to $g=\mathrm{D}^{V} \mathrm{D}^{V} E$, and consider the energy function $\widetilde{E}$ as defined by (6.21). Then, the corresponding canonical sprays $\Gamma$ and $\widetilde{\Gamma}$ are projectively equivalent, i.e. $\widetilde{\Gamma}=$ $\Gamma-2 \lambda \Delta$, if and only if $L$ satisfies

$$
g(\nabla L(X), Y)=\frac{1}{2}[g(X, \mathbf{T}) \alpha(Y)+g(Y, \mathbf{T}) \alpha(X)]
$$

where

$$
\alpha=-2 d_{L}^{V} \lambda \quad \text { and } \quad \lambda=\frac{1}{2} \operatorname{tr}\left(L \nabla L^{-1}\right) .
$$

Proof. We use the third of the equivalent conditions of theorem 22 with the role of $\nabla$ and $\widetilde{\nabla}$ interchanged, which is to say that we will characterise projective equivalence by the existence of a function $\lambda$ such that

$$
\nabla \widetilde{g}(X, Y)=\left(\mathrm{D}_{X}^{V} \lambda\right) \widetilde{g}(Y, \mathbf{T})+\left(\mathrm{D}_{Y}^{V} \lambda\right) \widetilde{g}(X, \mathbf{T})+2 \lambda \widetilde{g}(X, Y) .
$$

From the defining relation of $\widetilde{g}$, we obtain with the aid of (5.43) that

$$
\nabla \widetilde{g}(X, Y)=(\operatorname{det} L)^{-1}\left(\operatorname{tr}\left(L \nabla L^{-1}\right) g\left(L^{-1} X, Y\right)-g\left(\nabla L\left(L^{-1} X\right), L^{-1} Y\right)\right) .
$$


Observe in addition that for any Euler-Lagrange field, the connection coefficients have the property $\Gamma_{i}^{i}=\frac{1}{2} \Gamma(\log \operatorname{det} g)$. Indeed, we have $\nabla g=0$ or $\Gamma_{i}^{k} g_{k j}+\Gamma_{j}^{k} g_{i k}=\Gamma\left(g_{i j}\right)$, from which the result follows by multiplication with $g^{i j}$ and using (5.43).

Suppose now first that $\Gamma$ and $\widetilde{\Gamma}$ are projectively equivalent, i.e. $\widetilde{\Gamma}=\Gamma-2 \lambda \Delta$. Then $\nabla \widetilde{g}$, as given by (6.25), satisfies (6.24), yielding

$$
\begin{aligned}
\left(\operatorname{tr}\left(L \nabla L^{-1}\right)-2 \lambda\right) & g\left(L^{-1} X, Y\right)=g\left(\nabla L\left(L^{-1} X\right), L^{-1} Y\right) \\
+ & \left(\mathrm{D}_{X}^{V} \lambda\right) g\left(L^{-1} Y, \mathbf{T}\right)+\left(\mathrm{D}_{Y}^{V} \lambda\right) g\left(L^{-1} X, \mathbf{T}\right) .
\end{aligned}
$$

But the formula (6.13) for $\lambda$ here becomes (since both sprays are EulerLagrange fields)

$$
\lambda=\frac{1}{n+1}\left(\widetilde{\Gamma}_{i}^{i}-\Gamma_{i}^{i}\right)=\frac{1}{2(n+1)}(\widetilde{\Gamma}(\log \operatorname{det} \widetilde{g})-\Gamma(\log \operatorname{det} g)),
$$

and since $\log \operatorname{det} \widetilde{g}$ is homogeneous of degree 0 , we have $\widetilde{\Gamma}(\log \operatorname{det} \widetilde{g})=$ $\Gamma(\log \operatorname{det} \widetilde{g})$. It follows, with the aid of (6.19), that

$$
\begin{aligned}
\lambda & =\frac{1}{2} \Gamma\left(\log \left(\frac{\operatorname{det} \widetilde{g}}{\operatorname{det} g}\right)^{1 /(n+1)}\right) \\
& =\frac{1}{2} \Gamma\left(\log (\operatorname{det} L)^{-1}\right)=\frac{1}{2} \operatorname{tr}\left(L \nabla L^{-1}\right) .
\end{aligned}
$$

Hence, the left-hand side in (6.26) vanishes and renaming $L^{-1} X$ and $L^{-1} Y$ as $X$ and $Y$, we get the desired result (6.22) with $\alpha$ as in (6.23).

Conversely, if $L$ satisfies (6.22) with $\alpha$ as in (6.23), then (6.25) becomes

$$
\begin{aligned}
\nabla \widetilde{g}(X, Y)= & \operatorname{tr}\left(L \nabla L^{-1}\right) \widetilde{g}(X, Y)+\frac{1}{2} \mathrm{D}_{Y}^{V}\left(\operatorname{tr}\left(L \nabla L^{-1}\right)\right) \widetilde{g}(X, \mathbf{T}) \\
& +\frac{1}{2} \mathrm{D}_{X}^{V}\left(\operatorname{tr}\left(L \nabla L^{-1}\right)\right) \widetilde{g}(Y, \mathbf{T}) .
\end{aligned}
$$

So, there exists a $\lambda$, namely $\lambda=\frac{1}{2} \operatorname{tr}\left(L \nabla L^{-1}\right)$, such that condition (6.24) is satisfied, which means that $\Gamma$ and $\widetilde{\Gamma}$ are projectively equivalent.

Notice that condition (6.22) already has appeared in (5.22). It is the generalisation of the equation for special conformal Killing tensors (3.56) in the Riemannian case, where $\alpha=d f$ with $f$ the trace of the basic tensor $L$. Remark that expression (6.23) is automatically satisfied in that case. It's a consequence of the vanishing of the Nijenhuis torsion. Namely, $N_{L}=0$ means that

$$
L_{j \mid k}^{m} L_{i}^{k}-L_{k}^{m} L_{j \mid i}^{k}-L_{i \mid k}^{m} L_{j}^{k}+L_{k}^{m} L_{i \mid j}^{k}=0
$$


contracting this with $\left(L^{-1}\right)_{m}^{j}$, we find $\left(L^{-1}\right)_{m}^{j} L_{j \mid k}^{m} L_{i}^{k}=L_{k \mid i}^{k}$ or $\operatorname{tr}\left(L^{-1} \mathrm{D}_{L X}^{H} L\right)=$ $\operatorname{tr}\left(\mathrm{D}_{X}^{H} L\right)$ for an arbitrary vector field $X$. So, using the commutator property $\left[\mathrm{D}_{X}^{V}, \nabla\right]=\mathrm{D}_{X}^{H}-\mathrm{D}_{\nabla X}^{V}$ we get

$$
\mathrm{D}_{L X}^{V}\left(\operatorname{tr}\left(L^{-1} \nabla L\right)\right)=\operatorname{tr}\left(L^{-1} \mathrm{D}_{L X}^{H} L\right)=\operatorname{tr}\left(\mathrm{D}_{X}^{H} L\right)=d \operatorname{tr} L
$$

because $L$ is basic.

In the case of a Finsler metric and a type $(1,1)$ tensor $L$ along $\tau$, homogeneous of degree 0 , condition (6.23) will not automatically hold. But it is possible to write it in another form. Therefore, we introduce first a vector field $X_{\alpha} \in \mathcal{X}(\tau)$, defined by

$$
\left.X_{\alpha}\right\lrcorner g=\alpha .
$$

Now we can rewrite condition $(6.22)$ as an equality for type $(1,1)$ tensors:

$$
\nabla L=\frac{1}{2}\left(\mathbf{T} \otimes \alpha+X_{\alpha} \otimes \theta_{E}\right) .
$$

It has the same form as expression (5.22) where we replace $\alpha_{1}$ by $\alpha$ and $A_{1}$ by $-X_{\alpha}$. Also here (see (5.21) and (5.42)) we have that $\alpha(\mathbf{T})=\nabla \operatorname{tr} L$ and

$$
\alpha\left(L^{-1} \mathbf{T}\right)=\operatorname{tr}\left(L^{-1} \nabla L\right) .
$$

So, requirement (6.23) becomes

$$
\alpha(X)=\mathrm{D}_{L X}^{V}\left(\alpha\left(L^{-1} \mathbf{T}\right)\right)
$$

Setting $X=\mathbf{T}$, we find that

$$
\alpha(\mathbf{T})=\mathrm{D}_{L \mathbf{T}}^{V}\left(\alpha\left(L^{-1} \mathbf{T}\right)\right)
$$

which is the same as saying that

$$
\nabla \operatorname{tr} L=\mathrm{D}_{L \mathbf{T}}^{V} \operatorname{tr}\left(L^{-1} \nabla L\right)
$$

Inspired by what happens in the Riemannian case, we wonder if the Nijenhuistype tensors of $L$ can help to eliminate or simplify condition (6.23). This question will be discussed in the next section.

\subsection{Nijenhuis torsion condition on $L$}

As already said before, the special conformal Killing tensors have automatically vanishing Nijenhuis torsion and the same is true for their complete 
lift. The present situation however, is much more complicated. First of all, one can for every type $(1,1)$ tensor field $W$ along $\tau$, consider two Nijenhuistype tensors, namely $N_{W}^{V}$ and $N_{W}^{H}$ (see the definitions (4.1) and (4.2)). For our tensor $L$ it is not clear which Nijenhuis-type tensor should be relevant. Therefore we will investigate further the conditions arising from proposition 34 and theorem 23.

With $\widetilde{g}$ as before defined by (6.18), we now look again at the condition (6.20), which will guarantee that $\widetilde{g}=\mathrm{D}^{V} \mathrm{D}^{V} \widetilde{E}$ with $\widetilde{E}$ as in (6.21). Note first that by putting $X=L P$ and switching derivatives from $L^{-1}$ to $L,(6.20)$ can equivalently be written as

$$
\begin{aligned}
& \operatorname{tr}\left(\mathrm{D}_{Z}^{V} L \circ L^{-1}\right) g(P, Y)+g\left(\mathrm{D}_{Z}^{V} L(P), L^{-1} Y\right) \\
& \quad=\operatorname{tr}\left(\mathrm{D}_{Y}^{V} L \circ L^{-1}\right) g(P, Z)+g\left(\mathrm{D}_{Y}^{V} L(P), L^{-1} Z\right) .
\end{aligned}
$$

Putting next $Y=L Q, Z=L S$ and renaming subsequently $P, Q, S$ as $X, Y, Z$ again, this can further be written as

$$
\begin{aligned}
\operatorname{tr}\left(\mathrm{D}_{L Z}^{V} L \circ L^{-1}\right) & g(X, L Y)+g\left(\mathrm{D}_{L Z}^{V} L(X), Y\right) \\
& =\operatorname{tr}\left(\mathrm{D}_{L Y}^{V} L \circ L^{-1}\right) g(X, L Z)+g\left(\mathrm{D}_{L Y}^{V} L(X), Z\right) .
\end{aligned}
$$

Taking now $X=L Y=\mathbf{T}$, it follows from the homogeneity of $L$, i.e. the property $\mathrm{D}_{\mathbf{T}}^{V} L=0$, that

$$
2 E \operatorname{tr}\left(\mathrm{D}_{L Z}^{V} L \circ L^{-1}\right)+g\left(\mathrm{D}_{L Z}^{V} L(\mathbf{T}), L^{-1} \mathbf{T}\right)=0,
$$

or

$$
2 E \operatorname{tr}\left(\mathrm{D}_{L Z}^{V} L \circ L^{-1}\right)+g\left(N_{L}^{V}(Z, \mathbf{T}), L^{-1} T\right)+g\left(\mathrm{D}_{Z}^{V} L(\mathbf{T}), \mathbf{T}\right)=0
$$

Using this, (6.32) can be written in the form

$$
\begin{aligned}
& -g\left(L^{-1} N_{L}^{V}(Z, \mathbf{T})+\mathrm{D}_{Z}^{V} L(\mathbf{T}), \mathbf{T}\right) g(X, L Y)+2 E g\left(\mathrm{D}_{L Z}^{V} L(X), Y\right) \\
& \quad=-g\left(L^{-1} N_{L}^{V}(Y, \mathbf{T})+\mathrm{D}_{Y}^{V} L(\mathbf{T}), \mathbf{T}\right) g(X, L Z)+2 E g\left(\mathrm{D}_{L Y}^{V} L(X), Z\right)
\end{aligned}
$$

which is the same as

$$
\begin{aligned}
& 2 E\left(g\left(N_{L}^{V}(Z, X), Y\right)+g\left(\mathrm{D}_{Z}^{V} L(X), L Y\right)-g\left(\mathrm{D}_{L Y}^{V} L(X), Z\right)\right) \\
&=\quad g(X, L Y) g\left(L^{-1} N_{L}^{V}(Z, \mathbf{T})+\mathrm{D}_{Z}^{V} L(\mathbf{T}), \mathbf{T}\right) \\
&-g(X, L Z) g\left(L^{-1} N_{L}^{V}(Y, \mathbf{T})+\mathrm{D}_{Y}^{V} L(\mathbf{T}), \mathbf{T}\right) .
\end{aligned}
$$

This reformulation of the condition for having a second Finsler metric strongly suggests that a natural further assumption to adopt about $L$ is that $N_{L}^{V}=0$. 
Lemma 11. If a general, non-singular type $(1,1)$ tensor field $L$ along $\tau$ has the property $N_{L}^{V}=0$, then

$$
d_{L}^{V}(\log \operatorname{det} L)=d^{V}(\operatorname{tr} L) .
$$

Proof. It follows from $N_{L}^{V}=0$ that $\mathrm{D}_{Z}^{V} L=L^{-1} \circ \mathrm{D}_{L Z}^{V} L$. Hence

$$
\operatorname{tr}\left(\mathrm{D}_{L Z}^{V} L \circ L^{-1}\right)=\operatorname{tr}\left(\mathrm{D}_{Z}^{V} L\right)=\mathrm{D}_{Z}^{V}(\operatorname{tr} L),
$$

for arbitrary $Z$. Using property (5.43), one obtains equation (6.35).

Lemma 12. If $L$ is a symmetric non-singular type $(1,1)$ tensor homogeneous of degree 0 and has zero vertical covariant Nijenhuis tensor $N_{L}^{V}$, the condition (6.20) for $\widetilde{g}$, as defined by (6.18), to be a Finsler metric, is equivalent to the two requirements

$$
\begin{gathered}
2 E \mathrm{D}_{Z}^{V} \operatorname{tr} L=-g\left(\mathrm{D}_{Z}^{V} L(\mathbf{T}), \mathbf{T}\right) \\
2 E\left(g\left(\mathrm{D}_{Z}^{V} L(X), L Y\right)-g\left(\mathrm{D}_{L Y}^{V} L(X), Z\right)\right) \\
\quad=g(X, L Y) g\left(\mathrm{D}_{Z}^{V} L(\mathbf{T}), \mathbf{T}\right)-g(X, L Z) g\left(\mathrm{D}_{Y}^{V} L(\mathbf{T}), \mathbf{T}\right)
\end{gathered}
$$

Proof. As seen in the proof of lemma 11, it follows from $N_{L}^{V}=0$ that $\operatorname{tr}\left(\mathrm{D}_{L Z}^{V} L \circ L^{-1}\right)=\mathrm{D}_{Z}^{V}(\operatorname{tr} L)$. So equation (6.33) becomes

$$
2 E \mathrm{D}_{Z}^{V} \operatorname{tr} L=-g\left(\mathrm{D}_{Z}^{V} L(\mathbf{T}), \mathbf{T}\right) .
$$

Condition (6.37) follows immediately from (6.34) if $N_{L}^{V}=0$.

Coming back now to the problem at the end of section 6.4 , we find the following simplification

Proposition 35. If $L$ is a symmetric type (1,1) tensor field along $\tau$, homogeneous of degree 0 which satisfies (6.29) and $N_{L}^{V}=0$, then condition (6.23) is equivalent to $d_{L}^{V} \alpha=0$.

Proof. Suppose that $\alpha=d_{L}^{V} \operatorname{tr}\left(L^{-1} \nabla L\right)$. Because $N_{L}^{V}=0$, we have that $d_{L}^{\vee 2}=0$, so $d_{L}^{V} \alpha$ vanishes.

Conversely, assume that $d_{L}^{V} \alpha=0$. The vanishing of $N_{L}^{V}$ implies then that $\alpha=d_{L}^{V} \lambda^{\prime}$ for a function $\lambda^{\prime}$ homogeneous of degree 1. Further we have that

$$
\operatorname{tr}\left(L^{-1} \nabla L\right) \stackrel{(6.30)}{=} \alpha\left(L^{-1} \mathbf{T}\right)=\mathrm{D}_{\mathbf{T}}^{V} \lambda^{\prime}=\lambda^{\prime}
$$


So, $\alpha=d_{L}^{V} \operatorname{tr}\left(L^{-1} \nabla L\right)$.

The assumption $N_{L}^{V}=0$ seems also natural if we let $L$ play the role of the $K$-tensor coming from a tensor $J$, as described in the chapters 4 and 5 . In that situation, the condition $N_{K}^{V}=0$ is necessary for obtaining a tensor field $R$ with vanishing Nijenhuis torsion (see condition (4.40)). In the case that $L$ has to come from a tensor $J$, we have to adopt a supplementary restriction to $L$, namely

$$
\left.g\left(\mathrm{D}_{X}^{V} L(Y), Z\right)\right)=g\left(\mathrm{D}_{Z}^{V} L(Y), X\right)
$$

for all $X, Y, Z \in \mathcal{X}(\tau)$ (see (4.9)). Using lemma 12 we can prove the following equivalence.

Corollary 3. For a type $(1,1)$ tensor $L$, homogeneous of degree 0 and satisfying conditions (6.36) and (6.37), the requirement

$$
g\left(\mathrm{D}_{Z}^{V} L(X), Y\right)=g\left(\mathrm{D}_{Y}^{V} L(X), Z\right)
$$

is equivalent to the condition: $\operatorname{tr} L$ is basic.

Note that, as a result of (6.35), if $\operatorname{tr} L$ is basic then so is $\operatorname{det} L$. In fact, the traces of all powers of $L$ will be basic in such a case, which can be proven along the following lines. Note first that for all powers $m$ :

$$
\mathrm{D}_{L^{m} X}^{V} L=L^{m} \circ \mathrm{D}_{X}^{V} L, \quad \text { and } \quad \mathrm{D}_{L X}^{V} L^{m}=L \circ \mathrm{D}_{X}^{V} L^{m} .
$$

The first is a trivial consequence of $N_{L}^{V}=0$, the second is easily shown by induction. Using these properties one subsequently finds that

$$
N_{L}^{V}=0 \quad \Rightarrow \quad N_{L^{k}}^{V}=0, \quad \forall k .
$$

Finally, starting from $N_{L^{k}}^{V}=0$ and proceeding by induction again, we have

$$
\begin{aligned}
\mathrm{D}_{X}^{V} L^{k} & =L^{-k} \circ \mathrm{D}_{L^{k} X}^{V} L^{k} \\
& =L^{-(k-1)}\left(L^{-1} \circ \mathrm{D}_{L^{k} X}^{V} L \circ L^{k-1}+\mathrm{D}_{L^{k} X}^{V} L^{k-1}\right),
\end{aligned}
$$

from which it follows that

$$
\mathrm{D}_{X}^{V}\left(\operatorname{tr} L^{k}\right)=\operatorname{tr}\left(\mathrm{D}_{X}^{V} L^{k}\right)=\operatorname{tr}\left(L^{-1} \circ \mathrm{D}_{L^{k} X}^{V} L\right)+\operatorname{tr}\left(L^{-(k-1)} \circ \mathrm{D}_{L^{k-1}(L X)}^{V} L^{k-1}\right) .
$$

The first term on the right is zero if $\operatorname{tr} L$ is basic, while the second is $\operatorname{tr}\left(\mathrm{D}_{L X}^{V} L^{k-1}\right)$ and is zero by the induction hypothesis. 
Finally, one can note that the construction of first integrals as described in section 5.4 works also in the present situation where we consider a symmetric type (1,1) tensor $L$ satisfying (6.29). Only for the recursion formula in proposition $32, L$ has to satisfy two extra conditions: $L$ has to come from a tensor $J$ and $N_{L}^{V}=0$. In the present situation this means that the trace of $L$ has to be basic. The functions functions $a_{n}$ and $\phi_{n}$ (defined in (5.34) and (5.37)) are in this case also basic.

\subsection{Example with a Randers metric}

To find an illustrative example of the theory discussed in section 6.4, we make use of a property of Randers metrics. A Randers metric is a Finsler metric with a Finsler function $F$ of the form $F=\sqrt{a_{i j}(q) u^{i} u^{j}}+b_{i} u^{i}$ where $a_{i j}$ is a Riemann metric. It is known (see for example [45]) that the metric associated to $F$ is projectively equivalent with its corresponding Riemannian metric $a_{i j}$ if and only if the 1 -form $\beta=b_{i} d q^{i}$ is closed.

For simplicity, we start with the Finsler function $F$ of a free particle in two dimensions: $F=\sqrt{u_{1}^{2}+u_{2}^{2}}$ and we consider another function $\widetilde{F}=$ $\sqrt{u_{1}^{2}+u_{2}^{2}}+q_{2} u_{2}$. So the metrics corresponding to $F$ and $\widetilde{F}$ are projectively equivalent. The metric $\widetilde{g}$ is given by

$$
\begin{aligned}
& \widetilde{g}_{11}=1+\frac{q_{2} u_{2}^{3}}{\left(u_{1}^{2}+u_{2}^{2}\right)^{3 / 2}} \\
& \widetilde{g}_{12}=\frac{q_{2} u_{1}^{3}}{\left(u_{1}^{2}+u_{2}^{2}\right)^{3 / 2}} \\
& \widetilde{g}_{22}=1+q_{2}^{2}+\frac{3 q_{2} u_{2} u_{1}^{2}+2 q_{2} u_{2}^{3}}{\left(u_{1}^{2}+u_{2}^{2}\right)^{3 / 2}}
\end{aligned}
$$

and for the determinant of $\widetilde{g}$ we find

$$
\operatorname{det} \widetilde{g}=\left(1+\frac{q_{2} u_{2}}{\left(u_{1}^{2}+u_{2}^{2}\right)^{1 / 2}}\right)^{3} .
$$

The two metric tensors $g$ and $\widetilde{g}$ define a non-singular, symmetric tensor $L$, homogeneous of degree 0. Namely, making use of the formulas (6.18) and (6.19), we get that

$$
L=\widetilde{g}^{-1}\left(\frac{\operatorname{det} \widetilde{g}}{\operatorname{det} g}\right)^{1 / 3}=\widetilde{g}^{-1} \operatorname{det} \widetilde{g} \frac{u_{1}^{2}+u_{2}^{2}}{\left(\sqrt{u_{1}^{2}+u_{2}^{2}}+q_{2} u_{2}\right)^{2}} .
$$


So,

$$
\begin{aligned}
L_{1}^{1} & =\frac{\left(1+q_{2}^{2}\right)\left(u_{1}^{2}+u_{2}^{2}\right)^{3 / 2}+3 q_{2} u_{2} u_{1}^{2}+2 q_{2} u_{2}^{3}}{\widetilde{F}^{2} \sqrt{u_{1}^{2}+u_{2}^{2}}} \\
L_{2}^{1} & =-\frac{q_{2} u_{1}^{3}}{\widetilde{F}^{2} \sqrt{u_{1}^{2}+u_{2}^{2}}} \\
L_{2}^{2} & =\frac{\left(u_{1}^{2}+u_{2}^{2}\right)^{3 / 2}+q_{2} u_{2}^{3}}{\widetilde{F}^{2} \sqrt{u_{1}^{2}+u_{2}^{2}}}
\end{aligned}
$$

Now, we compute the dynamical covariant derivative $\nabla$ of $L$ (in this case $\nabla$ of a function $f$ is given by $\nabla(f)=\mathbf{T}(f), \nabla\left(\frac{\partial}{\partial q^{i}}\right)=0$ and $\left.\nabla\left(d q^{i}\right)=0\right)$ and we find

$$
\begin{aligned}
& (\nabla L)_{1}^{1}=\left[q_{2} u_{2} u_{1}^{2}\left(2 u_{1}^{2}+u_{2}^{2}\right)+u_{1}^{2} u_{2}^{2} \sqrt{u_{1}^{2}+u_{2}^{2}}\right] /\left[\widetilde{F}^{3} \sqrt{u_{1}^{2}+u_{2}^{2}}\right] \\
& (\nabla L)_{2}^{2}=-\left[q_{2} u_{2}^{5}+\left(u_{2}^{4}+2 u_{1}^{2} u_{2}^{2}\right) \sqrt{u_{1}^{2}+u_{2}^{2}}\right] /\left[\widetilde{F}^{3} \sqrt{u_{1}^{2}+u_{2}^{2}}\right] \\
& (\nabla L)_{2}^{1}=\left[q_{2} u_{1}^{3} u_{2}^{2}-u_{1}^{3} u_{2} \sqrt{u_{1}^{2}+u_{2}^{2}}\right] /\left[\widetilde{F}^{3} \sqrt{u_{1}^{2}+u_{2}^{2}}\right] .
\end{aligned}
$$

So, $\nabla L$ satisfies the fundamental relation (6.29), namely we have that $\nabla L_{j}^{i}=$ $\frac{1}{2}\left(\alpha_{j} u^{i}+\alpha_{i} u^{j}\right)$ for $i, j=1,2$ with

$$
\alpha_{1}=\left[q_{2} u_{2} u_{1}\left(2 u_{1}^{2}+u_{2}^{2}\right)+u_{1} u_{2}^{2} \sqrt{u_{1}^{2}+u_{2}^{2}}\right] /\left[\widetilde{F}^{3} \sqrt{u_{1}^{2}+u_{2}^{2}}\right]
$$

and

$$
\alpha_{2}=-\left[q_{2} u_{2}^{4}+\left(u_{2}^{3}+2 u_{1}^{2} u_{2}\right) \sqrt{u_{1}^{2}+u_{2}^{2}}\right] /\left[\widetilde{F}^{3} \sqrt{u_{1}^{2}+u_{2}^{2}}\right] .
$$

For this example, however, the vertical Nijenhuis tensor $N_{L}^{V}$ is not zero.

Starting from this type $(1,1)$ tensor $L$ which satisfies $(6.29)$, we can construct first integrals of the system $F$, as in section 5.4. This leads again to complicated calculations. Moreover, in the case of a free particle it is not an interesting problem and therefore we will not pursue this further. 


\section{Bibliography}

[1] S. Benenti, Inertia tensors and Stäckel systems in euclidean spaces, Rend. Sem. Mat. Univ. Polit. Torino 50 (1992) 315-341

[2] S. Benenti, Intrinsic characterization of variable separation in the Hamilton-Jacobi equation, J. Math. Phys. 38 (1997) 6578-6602

[3] S. Benenti, Special Symmetric Two-tensors, Equivalent Dynamical Systems, Cofactor and Bi-Cofactor Systems, Acta Appl. Math. 87 (2005) $33-91$

[4] M. Blaszak, Separable systems with quadratic in momenta first integrals, J. Phys. A: Math. Gen. 38 (2005) 1667-1685

[5] A. V. Bolsinov, V. S. Matveev, Geometrical interpretation of Benenti systems, J. Geom. Phys. 44 (2003) 489-506

[6] J. F. Cariñena, E. Martínez, A new geometric setting for Lax equations, Int. J. Modern Phys. A 9 (1994) 4973-4986

[7] M. Crampin, G. Marmo, C. Rubano, Conditions for the complete integrability of a dynamical system admitting alternative Lagrangians, Phys. Lett. A 97 (1983) 88-90

[8] M. Crampin, W. Sarlet, G. Thompson, Bi-differential calculi, biHamiltonian systems and conformal Killing tensors, J. Phys. A: Math. Gen. 33 (2000) 8755-8770

[9] M. Crampin, W. Sarlet, A class of non-conservative lagrangian systems on Riemannian manifolds, J. Math. Phys. 42-9 (2001) 4313-4326

[10] M. Crampin, Conformal Killing tensors with vanishing torsion and the separation of variables in the Hamilton-Jacobi equation, Diff. Geom. Appl. 18 (2003) 87-102

[11] M. Crampin, Projectively equivalent Riemannian spaces as quasi-biHamiltonian systems, Acta. Appl. Math. 77 (2003) 237-248 
[12] M. Crampin, On the orthogonal separation of variables int the Hamilton-Jacobi equation for geodesics in a Riemannian manifold, preprint

[13] M. De Léon, P. R. Rodriguez, Methods of Differential Geometry in Analytical Mechanics, North-Holland Mathematics Studies 158, Amsterdam (1989)

[14] A. Dimakis, F. Müller-Hoissen, Bi-differential calculi and integrable models, J. Phys. A: Math. Gen. 33 (2000) 957-974

[15] L.P. Eisenhart, Separable systems of Stäckel, Ann. Math. 35 (1934) 284-305

[16] A. Ibort, F. Magri and G. Marmo, Bihamiltonian structures and Stäckel separability, J. Geometry and Physics 33 (2000) 210-228

[17] G. Falqui, M. Pedroni, Separation of variables for bi-Hamiltonian systems, Math. Phys. Anal. Geom. 6 (2003) 139-179

[18] G. Falqui, Poisson pencils, Integrability and Separation of variables, to appear in Phil. Trans. Roy. Soc. A (2005)

[19] C. Ferrario, G. Lo Vecchio, G. Marmo, G. Morandi, Separability of completely integrable systems admitting alternative Lagrangian descriptions, Lett. math. Phys 9 (1985) 141-148

[20] C. Ferrario, G. Lo Vecchio,G. Marmo,G. Morandi,C. Rubano, A separability theorem for dynamical systems admitting alternative Lagrangian descriptions, J. Phys. A: Math. Gen. 20 (1987) 3225-3236

[21] A. Frölicher, A. Nijenhuis, Theory of vector-valued differential forms, Proc. Ned. Acad. Wetensch. Sér. A 59 (1956) 338-359

[22] S. Hojman, H. Harleston, Equivalent Lagrangians: multidimensional case, J. Math. Phys. 22 (1981) 1414-1419

[23] T. Levi-Civita, Sulla integrazione della equazione di Hamilton-Jacobi per sepa razione di variabili, Math. Ann. 59 (1904) 383-397

[24] Y. Kosmann-Schwarzbach, F. Magri, Poisson-Nijenhuis structures, Ann. Inst. Henri Poincaré, Phys. Théor. 53 (1990) 35-81

[25] D. Krupka, A. E. Sattarov, The inverse problem of the calculus of variations for Finsler structures, Math. Slovaca 3 (1985) 217-222 
[26] O. Krupková, Variational metric structures, Publ. Math. Debrecen 62 (2003) 461-495

[27] P. Libermann, C. M. Marle, Symplectic Geometry and analytical mechanics, D. Reidel Publishing Company, Dordrecht (1987)

[28] J. Liouville, L'intégration des equations différentielles du mouvement d'un nombre quelconque de points matérielles, J. Math. Pures Appl. 14 (1849) 275-299

[29] J. Liouville, Note sur les équations de la dynamique, J. Math. Pures Appl. 20 (1855) 137-138

[30] H. Lundmark, Higher-dimensional integrable Newton systems with quadratic integrals of motion, Stud. Appl. Math. 110-3 (2003) 257-296

[31] H. Lundmark, Multiplicative structure of cofactor pair systems in Riemannian spaces, preprint (2001)

[32] F. Magri, A simple model of integrable Hamiltonian equation, J. Math. Phys. 19 (1978) 1156-1162

[33] F. Magri, Eight lectures on integrable systems, Integrability of Nonlinear Systems, Springer (1996) 236-296

[34] F. Magri. A short introduction to Hamiltonian PDE's

[35] E. Martínez, Geometía de ecuaciones diferenciales aplicada al la Mecánica, Ph.D. Thesis, University of Zaragoza, Spain (1991)

[36] E. Martínez, J.F. Cariñena and W. Sarlet, Derivations of differential forms along the tangent bundle projection, Diff. Geometry and its Applications 2 (1992) 17-43

[37] E. Martínez, J.F. Cariñena and W. Sarlet, Derivations of differential forms along the tangent bundle projection II, Diff. Geometry and its Applications 3 (1993) 1-29

[38] E. Martínez, J.F. Cariñena and W. Sarlet, Geometric characterization of separable second-order equations, Math. Proc. Camb. Phil. Soc. 113 (1993) 205-224

[39] V. S. Matveev, P. I. Topalov, Trajectory equivalence and corresponding integrals, Regular and Chaotic Dynamics 3-2 (1998) 30-45 
[40] G. Mitric, I. Vaisman, Poisson structures on tangent bundles, Diff. Geom. Appl. 18 (2003) 207-228

[41] G. Morera, Sulla separazione delle variabili nelle equazioni del moto di un punto materiale su una superfice, Atti. Sci. di Torino 16 (1881) $276-295$

[42] P. J. Olver, Applications of Lie groups to differential equations, Springer-Verlag, New York (1986)

[43] D. Saunders, The geometry of Jet bundles, Cambridge University Press, Cambridge (1989)

[44] W. Sarlet, F. Vermeire, A class of Poisson-Nijenhuis structures on a tangent bundle, J. Phys. A: Math. Gen. 37 (2004) 6319-6336

[45] Z. Shen, Differential geometry of Spray and Finsler spaces, Kluwer Academic Publishers, Dordrecht (2001)

[46] E. K. Sklyanin, Separation of variables. New trends, Prog. Theor. Phys. Suppl. 118 (1995) 35-60

[47] P. Stäckel, Uber die integration der Hamilton-Jacobischen Differential Gleichung mittelst Separation der variabel, Habilitationsschrift, Halle (1891)

[48] J. Szilasi, Sz. Vattamány, On the Finsler-metrizabilities of spray manifolds, Period. Math. Hungarica 44-1 (2002) 81-100

[49] G. Tondo, bi-Hamiltonian manifolds, quasi-bi-Hamiltonian systems and separation variables, Rep. Math. Phys. 44-1 (1999) 225-266

[50] I. Vaisman, Locally Lagrangian symplectic and Poisson manifolds, Rend. Sem. Mat. Univ. Pol. Torino 59 (2001) 43-58

[51] I. Vaisman, Lagrange geometry on tangent manifolds, Int. J. Math. Math. Sci. 2003-51 (2003) 3241-3266

[52] F. Vermeire, W. Sarlet and M. Crampin, A class of recursion operators on a tangent bundle, J. Phys. A: Math. Gen. 39 (2006) 7319-7340 


\section{Index}

A-tensor, 30

Almost tangent structure, 47

canonical -, 42

Alternative Lagrangian, 72

Associated semispray, 114

Benenti systems, 26

Benenti tensor, 31

Berwald connection, 90

Bi-differential calculus

gauged -, 20

simple -, 19

Bi-Hamiltonian

manifold, 11

vector field, 11

Canonical spray, 90

Casimir, 8

Characteristic, 15

Characteristic Killing tensor, 26

Cofactor

pair systems, 35

system, 34

Cofactor tensor, 32

Complete lift, 47

Control matrix, 26

Covariant derivative

dynamical -, 44

horizontal -, 43

vertical,- 43

Covariant Nijenhuis tensor

horizontal,- 65

vertical,- 65

Darboux-Nijenhuis coordinates, 20
Deviation, 46

Differential function, 14

Elliptic coordinates matrices, 31

Evolutionary vector field, 15

Finsler function, 89

Finsler manifold, 89

Finsler metrizability in broad sense, 117

Frölicher-Nijenhuis calculus, 77

Generalised

infinitesimal symmetry, 15

vector field, 14

Geodesic spray, 90

Geodesically equivalent, 115

Haantjes tensor, 53

Hamilton-Jacobi, 21

Hamiltonian vector field, 8

Harmonic oscillator, 82

Helmholtz conditions, 45

Horizontal lift, 42 along the projection, 112

Integrable, 8

Involution, 8

Jacobi endomorphism, 44

Jet manifold, 14

Killing

conformal - tensor, 30

tensor, 25, 29

vector, 29 
Killing-Stäckel system, 25

Korteweg De Vries

equation, 9

hierarchy, 12

Lenard's recursion relations, 12

Linear connection along the tangent bundle projection, 110

Liouville

systems, 22

Magri-Morosi concomitant, 18

Miura transformation, 11

Mixed curvature tensor, 43

Nijenhuis operator, 17

Nijenhuis torsion, 17

Parallel, 112

Pencil, 11

Planar inertia tensors, 31

Poisson

bracket, 7

manifold, 7

map, 8

tensor, 8

Poisson-Nijenhuis manifold, 17

Projective equivalence, 37

Projectively equivalent, 115

Quasi-Hamiltonian, 34, 38

bi- -, 35

R-tensor, 18

Randers metric, 125

Recursion operator, 15, 17

Semisimple, 20

Separable, 21

orthogonally,- 21

Solitons, 9

Source projection, 14
Special conformal Killing tensor, 26

Spray, 114 space, 115

Stäckel

matrix, 22

separable, 23

system, 23

Target projection, 14

Total time derivative, 42

Vertical endomorphism, 42

Vertical lift, 42

Vertical representative, 15 


\section{Samenvatting}

Het algemeen referentiekader voor deze thesis, is dat van integreerbare Hamiltoniaanse systemen. Het onderwerp is ontstaan met een beroemde stelling van Liouville in de Hamiltoniaanse mechanica en is nog steeds populair. Een interessante deelklasse binnen deze systemen bestaat uit de systemen met een bi-Hamiltoniaans karakter: ze zijn Hamiltoniaans ten opzichte van twee Poissonstructuren die compatibel zijn, i.e. waarvan de lineaire combinatie ook een Poissonstructuur is. Deze bi-Hamiltoniaanse structuur is voor het eerst ontdekt in 1978 door F. Magri in het geval van evolutievergelijkingen, zoals de bekende Korteweg-De Vriesvergelijking. Men kan aantonen dat de interactie tussen de twee Poissonstructuren aanleiding geeft tot een hiërarchie van eerste integralen die in involutie zijn voor beide haakjes van Poisson. Deze theorie wordt geschetst in het eerste hoofdstuk van deze verhandeling. We beschrijven ook kort de systemen die scheidbaar zijn in de zin van Hamilton-Jacobi, in het bijzonder de zogenaamde Stäckelscheidbaarheid en de Eisenhartvoorwaarden.

Een Poisson-Nijenhuisvariëteit bestaat uit een Poissontensor $P$ en een $(1,1)$ tensorveld $R$ waarvoor geldt dat $P R=R P$ een tweede compatibel Poissonhaakje definieert. Zo'n variëteit heeft dus een bi-Hamiltoniaanse structuur en de tensor $R$ wordt de recursieoperator genoemd. Uit de compatibiliteit van de twee Poissonhaakjes volgt dan dat de Nijenhuiswringing van $R$ verdwijnt.

Een bekend voorbeeld van zo'n Poisson-Nijenhuisstructuur ontstaat als volgt. Beschouw een $(1,1)$-tensor $J$ op een gegeven Riemannvariëteit. De canonische symplectische structuur $\omega$ op de co-raakbundel bepaalt reeds een eerste Poissonhaakje. Met behulp van de tensor $J$ construeren we een tweede 2vorm $\omega_{1}=\mathcal{L}_{J^{v}} \omega($ zie $(2.8))$. De relatie

$$
i_{R(\xi)} \omega=i_{\xi} \omega_{1}
$$

met $\xi$ een willekeurig vectorveld op de co-raakbundel, definieert dan een tensor $R$ (een $R$-tensor genoemd). In feite is $R$ hier de complete lift van 
$J$ naar de co-raakbundel. Als de Nijenhuiswringing van $J$ verdwijnt, verdwijnt ook deze van $R$ en $R$ is dan de recursieoperator van de PoissonNijenhuisstructuur bepaald door $\omega$ en $J$.

Men heeft onder andere onderzocht onder welke omstandigheden de zonet gedefinieerde tensor $R$ invariant is. Maar onze interesse gaat vooral uit naar een speciale klasse van niet-invariante $\mathrm{R}$-tensoren die verbonden zijn met het bestaan van een zogenaamde geijkte bi-differentiale calculus. Want deze klasse van R-tensoren legt een interessante voorwaarde op aan de tensor $J$. Deze moet dan namelijk t.o.v de beschouwde Riemannmetriek $g$ voldoen aan een relatie van de vorm

$$
J_{i j \mid k}=\frac{1}{2}\left(g_{j k} \alpha_{i}+g_{i k} \alpha_{j}\right)
$$

met $\mid k$ de covariante afgeleide, $\alpha_{i}=\frac{\partial f}{\partial q^{i}}$ en $f$ het spoor van $J$. Voor zo'n tensor $J$ geldt dat

$$
J_{i j \mid k}+J_{j k \mid i}+J_{k i \mid j}=g_{i j} \alpha_{k}+g_{j k} \alpha_{i}+g_{k i} \alpha_{j},
$$

m.a.w $J$ is een conforme Killingtensor. Aangezien niet alle conforme Killingtensoren deze vorm hebben, spreekt men van een speciale conforme Killingtensor. Als zo'n tensor enkelvoudige eigenwaarden heeft, wordt hij ook wel een Benentitensor genoemd, naar S. Benenti die deze klasse van tensoren als eerste bestudeerde.

In hoofdstuk 2 van deze thesis bespreken we kort dit type conforme Killingtensoren. We herhalen de definitie en enkele interessante eigenschappen. De belangrijkste eigenschap is wel dat de Nijenhuiswringing van een speciale conforme Killingtensor automatisch verdwijnt. Verder geldt er dat de cofactortensor van zo een niet-singuliere tensor $J$ een Killingtensor is, m.a.w. een kwadratische eerste integraal van het systeem bepaalt. Als $J$ een speciale conforme Killingtensor is, dan is $J+s I$ dat ook, voor elke willekeurige constante $s$. De coëfficiënten van de verschillende machten van $s$ van de cofactortensor van $J+s I$, bepalen dan een verzameling van eerste integralen ([10]). Bovendien voldoen ze aan de Eisenhartvoorwaarden.

In hetzelfde hoofdstuk bespreken we ook enkele andere onderzoeksdomeinen waarin de speciale conforme Killingtensoren opduiken. Een eerste voorbeeld is te vinden in de theorie van de zogenaamde cofactorsystemen en cofactorpaarsystemen. Deze is ontwikkeld door H. Lundmark [30] op een Euclidische ruimte en is dan uitgebreid tot Riemannvariëteiten door M. Crampin en W. Sarlet [9]. Daar worden zo'n systemen in verband gebracht met 
niet-conservatieve Lagrangiaanse systemen die een quasi-Hamiltoniaanse representatie hebben ten opzichte van het Poissonhaakje komende van een (1,1)-tensor $L$. Het is daarvoor nodig dat $L$ een speciale conforme Killingtensor is. Een andere belangrijke toepassing voor dit soort tensoren vinden we in de studie van projectief of geodetisch equivalente Riemannmetrieken, i.e. metrieken die dezelfde geodeten hebben op een herparametrisatie na. M. Crampin heeft aangetoond dat de (1,1)-tensor $L$ die twee projectief equivalente metrieken $g$ en $\widetilde{g}$ aan elkaar linkt, en meer bepaald gedefinieerd is als:

$$
L_{i j}=\left(\frac{\operatorname{det} \widetilde{g}}{\operatorname{det} g}\right)^{1 / n+1} \widetilde{g}_{i j}^{-1}
$$

een speciale conforme Killingtensor is. Het omgekeerde is ook waar: als $L$ een niet-singuliere speciale conforme Killingtensor t.o.v een Riemannmetriek $g$ is, dan zullen $g$ en $\widetilde{g}$, gedefinieerd door

$$
\widetilde{g}_{i j}=(\operatorname{det} L)^{-1} L_{i j}^{-1}
$$

projectief equivalent zijn (zie [11]).

Tot hier de herhaling van de reeds gekende resultaten. Het belangrijkste doel van ons onderzoek is nu het veralgemenen van de theorie van speciale conforme Killingtensoren op een Riemannvariëteit naar een algemene Lagrangiaanse variëteit, i.e. een raakbundelvariëteit waarop we een reguliere functie $\ell$ beschouwen. Bij deze uitbreiding vertrekken we ook niet langer van een (1,1)-tensor op de basisvariëteit. De $(1,1)$-tensor zal nu ook van de snelheden afhangen. In het bijzonder beschouwen we ook een Lagrangiaan komende van een Finslermetriek. In dit geval zal de snelheidsafhankelijkheid van de (1,1)-tensor homogeen van de graad nul zijn.

Als voorbereiding op dit onderzoek, hebben we eerst van naderbij bekeken hoe de theorie van Poisson-Nijenhuisstructuren (besproken in hoofdstuk 2), die ontwikkeld werd op een co-raakbundel, op een natuurlijke manier kan bekomen worden met raakbundeltechnieken. Dit wordt besproken in het derde hoofdstuk. Eerst herhalen we de voornaamste ingrediënten van de calculus langs de raakbundelprojectie $\tau$ zoals die ontwikkeld is in [36] en [37]. Het vertrekpunt hierbij is een connectie op de raakbundel zodat een horizontale lift $X^{H}$ van een vectorveld langs $\tau$ naar de raakbundel kan worden geconstrueerd. Verder definieert men volgende afleidingoperatoren van differentiaalvormen langs $\tau$ : de horizontale en verticale uitwendige afgeleide $d^{H}$ en $d^{V}$ en de horizontale en verticale covariante afgeleide $\mathrm{D}^{H}$ en $\mathrm{D}^{V}$. In het geval dat de connectiecoëfficiënten symmetrisch zijn, m.a.w dat de connectie 
komt van een syteem van tweede-ordedifferentiaalvergelijkingen, hebben we nog twee andere belangrijke operatoren langs $\tau$ : de dynamisch covariante afgeleide $\nabla$ en het Jacobi-endomorfisme $\Phi$. In deze verhandeling bekijken we vooral de situatie dat het tweede-ordevectorveld bepaald wordt door de Euler-Lagrangevergelijkingen van een gegeven Lagrangiaan. Voor definities en meer details verwijzen we naar paragraaf 3.1.

Met behulp van deze calculus langs de projectie komen we tot een raakbundelversie van de gekende resultaten en sommige daarvan kunnen we direct uitbreiden tot het geval dat we beschikken over een willekeurige reguliere Lagrangiaan. Kort geschetst verlopen die constructies als volgt. Eenmaal de raakruimte voorzien is van een reguliere Lagrangiaan $\ell$ beschikken we over een geassocieerde symplectische 2 -vorm $\omega_{\ell}=d \theta_{\ell}=d(S d \ell)$ ). Vertrekkend van een (1,1)-tensor $J$ op de basisvariëteit, kunnen we aantonen dat $J^{c} S$ (met $J^{c}$ de complete lift van $J$ en $S$ het verticaal endomorfisme), net zoals $S$ een bijna-raakstructuur bepaalt. Bijgevolg is het natuurlijk om een tweede 2-vorm te construeren door in de bepalingsformule van $\omega_{\ell}, S$ te vervangen door $J^{c} S$. Met behulp van formule (1) definiëren we dan een tensor $R$. In hoofdstuk 3 bestuderen we de structuur van deze tensor en ook enkele eigenschappen. We vinden dat

$$
\begin{aligned}
& R\left(X^{V}\right)=(\bar{J} X)^{V} \\
& R\left(X^{H}\right)=(J X)^{V}+(U X)^{H}
\end{aligned}
$$

met $g(\bar{J} X, Y)=g(X, J Y)$ en $g(U X, Y)=d^{H}\left(J \theta_{\ell}\right)(X, Y)$ als $g$ de Hessiaan $\mathrm{D}^{V} \mathrm{D}^{V} \ell$ van de Lagrangiaan $\ell$ is.

Voor het specifieke geval dat $\ell$ een kinetische-energie Lagrangiaan, afkomstig van een Riemannmetriek is, onderzoeken we twee belangrijke situaties. We bewijzen dat $R$ invariant is onder de gegeven dynamica, m.a.w. dat $R$ een recursieoperator voor het genereren van symmetrieën is, als en slechts als $J$ symmetrisch en parallel is. We beschouwen ook het geval dat geldt

$$
d d_{R} \ell=d f \wedge d \ell
$$

met $f$ een functie op de basisvariëteit en $d_{R}$ gedefinieerd in paragraaf 1.2.2. De tensor $R$ is dan niet invariant terwijl $J$ opnieuw symmetrisch moet zijn en verder nog moet voldoen aan de volgende uitdrukking die een alternatieve, intrinsieke karakterisatie inhoudt van het speciaal conform Killing zijn:

$$
g(\nabla J(X), Y)=\frac{1}{2}\left[g(X, \mathbf{T}) \mathrm{D}_{Y}^{H} f+g(X, \mathbf{T}) \mathrm{D}_{X}^{H} f\right] .
$$


Hierin zijn $X$ en $Y$ willekeurige vectorvelden op de basisvariëteit, is $g$ de gegeven Riemannmetriek, $\nabla$ de dynamische covariante afgeleide en $\mathbf{T}=u^{i} \frac{\partial}{\partial q^{i}}$. We vinden verder een uitdrukking voor de tensor $U$ en de commutator van $J$ en het Jacobi-endomorfisme $\Phi$ in functie van $\alpha$.

Als we eenmaal goed begrijpen hoe deze constructies op de raakbundel werken, kunnen we beginnen aan de eigenlijke veralgemening. In hoofdstuk 4 vertrekken we dus van een Lagrangiaanse variëteit en een tensor $J$ langs de raakbundelprojectie. De bedoeling is om zo mogelijk, op analoge manier als in hoofdstuk 3, een tweede Poissonhaakje te construeren. Daarvoor merken we op dat $J^{c} S$ in feite gelijk is aan de verticale lift $J^{V}$ van $J$ en dat deze lift betekenis houdt wanneer $J$ een tensorveld langs $\tau$ is. Ook hier gebruiken we relatie (1) om een $\mathrm{R}$-tensor te bepalen. De structuur van deze veralgemeende tensor $R$ wordt gegeven in de volgende stelling.

Stelling 1. Het $(1,1)$ tensorveld $R$ op de raakbundel wordt gekarakteriseerd door

$$
\begin{gathered}
R\left(X^{V}\right)=(\bar{K} X)^{V} \\
R\left(X^{H}\right)=(K X)^{V}+(U X)^{H} \\
\text { met } g(K X, Y)=\mathrm{D}^{V}\left(J \theta_{\ell}\right)(X, Y) \text { en } g(U X, Y)=d^{H}\left(J \theta_{\ell}\right)(X, Y)
\end{gathered}
$$

Verrassend hierbij is dat in deze uitdrukkingen een tensor $K$ de plaats van de tensor $J$ inneemt. Deze tensor $K$ zal dan ook een belangrijke rol spelen in deze theorie. $K$ reduceert tot $J$ als $J$ onafhankelijk van de snelheden is en een andere belangrijke eigenschap, die we veelvuldig gebruiken in de bewijsvoering, is de volgende:

Stelling 2. Voor willekeurige vectorvelden $X, Y, Z \in \mathcal{X}(\tau)$ geldt:

$$
g\left(\mathrm{D}_{X}^{V} K(Y), Z\right)=g\left(\mathrm{D}_{Z}^{V} K(Y), X\right) .
$$

Als $K$ symmetrisch is, drukt deze eigenschap uit dat $K$ de Hessiaan van een zekere functie is. Onze aandacht gaat ook naar de recursie-eigenschappen van $R$. We onderzoeken wanneer $R$ invariant onder de stroming van het gegeven Lagrangiaans systeem is, hetgeen in direct verband kan worden gebracht met de theorie over alternatieve Lagrangianen. De tensor $R$ is in dit geval de horizontale lift van $K$. Verder bestuderen we ook de Nijenhuiswringing van $R$. Zoals verwacht, is het probleem hier heel wat complexer, maar er treden toch enkele interessante vereenvoudigingen op. De theorie wordt geïllustreerd aan de hand van een paar voorbeelden. 
In hoofdstuk 5 beschouwen we een Finslervariëteit $(Q, E)$ met canonische spray $\Gamma$ en Finsler metriek $g$. De tensor $J$ wordt homogeen van de graad nul in de snelheden verondersteld. De tensoren $K$ en $U$ zijn dan respectievelijk homogeen van de graad nul en één. In deze situatie vereenvoudigen de voorwaarden opdat $R$ invariant zou zijn.

Stelling 3. $\mathcal{L}_{\Gamma} R$ verdwijnt als en slechts als $K$ symmetrisch en parallel is.

$\mathrm{Na}$ heel wat ingewikkelde berekeningen komen we ook voor het Nijenhuiswringingsvrij zijn van $R$ tot twee eenvoudige voorwaarden.

Stelling 4. De Nijenhuiswringing $N_{R}$ van $R$ is nul als en slechts als

$$
\begin{gathered}
\mathcal{N}_{K}^{V}=0 \quad \text { en } \\
\mathcal{N}_{K}^{H}(X, Y)+\mathrm{D}_{U X}^{V} K(Y)-\mathrm{D}_{U Y}^{V} K(X)=0 .
\end{gathered}
$$

De definities van de Nijenhuis-type tensoren $\mathcal{N}_{W}^{V}$ en $\mathcal{N}_{W}^{H}$ voor een tensorveld $W$ kan men vinden in paragraaf 4.1 .

Als veralgemening van de uitdrukking (2) zijn we in eerste instantie uitgegaan van een relatie van de vorm

$$
d d_{R} E=\alpha \wedge d E
$$

met $\alpha=\alpha_{1}{ }^{H}+\alpha_{2}{ }^{V}$ een voorlopig willekeurige 1-vorm op de raakbundel. Dit leidt hier tot volgende voorwaarden:

Stelling 5. De tensor $R$ voldoet aan de relatie (5) als en slechts als

$$
\begin{gathered}
g(\bar{K}(X), Y)=g(K X, Y)-\alpha_{2}(X) g(Y, \mathbf{T})+\alpha_{2}(Y) g(X, \mathbf{T}) \\
g(\nabla K(X), Y)=\frac{1}{2}\left[\left(\alpha_{1}+\nabla \alpha_{2}\right)(X) g(Y, \mathbf{T})+\left(\alpha_{1}-\nabla \alpha_{2}\right) g(X, \mathbf{T})\right] \\
g(U X, Y)=-\frac{1}{2}\left[\left(\alpha_{1}-\nabla \alpha_{2}\right)(X) g(Y, \mathbf{T})-\left(\alpha_{1}-\nabla \alpha_{2}\right)(Y) g(X, \mathbf{T})\right] \\
g([\Phi, K] X, Y)=-\Phi \circ \alpha_{2}(X) g(Y, \mathbf{T})+\frac{1}{2}\left[\left(\nabla \alpha_{1}-\nabla^{2} \alpha_{2}\right)(X) g(Y, \mathbf{T})\right. \\
\left.-\left(\nabla \alpha_{1}-\nabla^{2} \alpha_{2}\right)(Y) g(X, \mathbf{T})\right]
\end{gathered}
$$

voor elke twee vectorvelden $X$ en $Y$ langs de raakbundelprojectie $\tau$.

Merk op dat in het klassieke Riemanngeval met een tensor $J$ op de basis, de laatste twee vergelijkingen automatisch uit de eerste twee volgen. Dit 
is hier echter niet het geval. Deze vergelijkingen leggen in zekere zin extra voorwaarden op aan de 1-vorm $\alpha$. We kunnen wel twee speciale situaties bespreken waarbij deze voorwaarden vereenvoudigen. In het geval dat $\alpha=d f$ met $f$ een functie op de raakbundel, is er automatisch aan (8) en (9) voldaan. $f$ is hier het spoor van de tensor $K$. Anderzijds bekijken we het geval dat $K$ symmetrisch verondersteld wordt. Dan vinden we onder andere dat $\nabla \alpha_{2}=0$ en $d^{V} \alpha_{1}=0$. Voorwaarde (7) wordt dan

$$
g(\nabla K(X), Y)=\frac{1}{2}\left[g(X, \mathbf{T}) \alpha_{1}(Y)+g(Y, \mathbf{T}) \alpha_{1}(X)\right]
$$

hetgeen dezelfde vorm heeft als (3). Analoog als bij de speciale conforme Killing tensor bepaalt de cofactortensor van een symmetrische tensor $K$ die voldoet aan (10), een eerste integraal van het systeem. We kunnen bovendien een ganse verzameling van niet-kwadratische eerste integralen construeren. Deze constructie kan ook hier gebeuren met behulp van de cofactortensor van $K+s I$. Maar in dit hoofdstuk presenteren we ook een andere werkwijze die als voordeel heeft dat $K$ niet regulier moet verondersteld worden. Een groot verschil tussen de theorie over speciale conforme Killingtensoren op een Riemannvariëteit en (1,1)-tensoren $K$ die voldoen aan (10) is dat de Nijenhuis-type tensoren $\left(\mathcal{N}_{K}^{V}\right.$ en $\left.\mathcal{N}_{K}^{H}\right)$ niet automatisch verdwijnen. Merk verder nog op dat als $K$ symmetrisch is en $\alpha=d f$, dan volgt uit (6) dat de functie $f$ onafhankelijk van de snelheden moet zijn.

Het laatste hoofdstuk van deze verhandeling, hoofdstuk 6, gaat over projectieve equivalentie. Eerst bekijken we in detail de constructie van een lineaire connectie langs de raakbundelprojectie vertrekkende van een bepaald tweede-ordevectorveld. We herhalen ook de definitie en enkele eigenschappen in verband met de projectieve equivalentie van twee sprays. In het bijzonder bestuderen we de projectieve equivalentie van twee Finslermetrieken. Daarvoor beschouwen we een Finslermetriek $g$ en een niet-singuliere symmetrische $(1,1)$ tensor $L$ langs de projectie, homogeen van de graad nul. De vraag is nu onder welke omstandigheden de $(0,2)$ tensor $\widetilde{g}$ gedefinieerd door

$$
\widetilde{g}(X, Y)=(\operatorname{det} L)^{-1} g\left(L^{-1} X, Y\right)
$$

een Finslermetriek projectief equivalent met de gegeven metriek $g$ is. We vinden dat $\widetilde{g}$ een Finsler metriek is als en slechts als $L$ voldoet aan

$$
\begin{aligned}
& \operatorname{tr}\left(L \mathrm{D}_{Z}^{V} L^{-1}\right) g\left(L^{-1} X, Y\right)+g\left(\left(\mathrm{D}_{Z}^{V} L^{-1}\right) X, Y\right) \\
& \quad=\operatorname{tr}\left(L \mathrm{D}_{Y}^{V} L^{-1}\right) g\left(L^{-1} X, Z\right)+g\left(\left(\mathrm{D}_{Y}^{V} L^{-1}\right) X, Z\right) .
\end{aligned}
$$

De nodige en voldoende voorwaarden opdat $\widetilde{g}$ projectief equivalent met $g$ zou zijn, worden gegeven in de volgende stelling. 
Stelling 6. Een Finslermetriek $\widetilde{g}$ gedefinieerd door (11) is projectief equivalent met $g$ als en slechts als

$$
g(\nabla L(X), Y)=\frac{1}{2}[g(X, \mathbf{T}) \alpha(Y)+g(Y, \mathbf{T}) \alpha(X)]
$$

met

$$
\alpha=-2 d_{L}^{V} \lambda \quad \text { en } \quad \lambda=\frac{1}{2} \operatorname{tr}\left(L \nabla L^{-1}\right) .
$$

In het Riemanngeval met een tensor $L$ en een 1-vorm op de basis, is deze laatste voorwaarde (14) automatisch vervuld als gevolg van het verdwijnen van de Nijenhuiswringing van $L$. We verwachten dus dat ook hier de Nijenhuis-type tensoren van $L$ de voorwaarde (14) zullen vereenvoudigen. Inderdaad, we vinden bijvoorbeeld dat, als $N_{L}^{V}=0,(14)$ equivalent is met eisen dat $d_{L}^{V} \alpha=0$.

Zowel in de context van R-tensoren, geassocieerd aan een $(1,1)$ tensor langs de raakbundelprojectie, als in de context van projectief equivalente Finslermetrieken treffen we dus eenzelfde voorwaarde aan (nl. (10) en (13)). Deze reduceert in het geval van een Riemannmetriek en een tensor op de basisvariëteit tot de 'speciale conforme Killing tensor'-voorwaarde (3) en kunnen we dus als veralgemening van dit concept beschouwen. Hoewel de Finslermeetkunde heel wat interesssante toepassingen kent (b.v. het vierde probleem van Hilbert, Zermelo's navigatieprobleem, toepassingen in de fysica en in de biologie,...), vindt men in deze verhandeling maar weinig voorbeelden. Vaak is het immers moeilijk om interessante gerelateerde voorbeelden op te stellen en vragen ze bovendien heel veel rekenwerk. Over het onderwerp van veralgemeende speciale conforme Killing tensoren is er dus nog heel wat meer onderzoek mogelijk, vooral dan op het gebied van toepassingen. 